\title{
Método de Propagação de Ondas para Leis de Conservação com Termo Forçante
}

\author{
Hernán Arquímedes Cuti Gutiérrez
}

\section{DISSERTAÇÃO APRESENTADA}

$\mathrm{AO}$

INSTITUTO DE MATEMÁTICA E ESTATÍSTICA

DA

UNIVERSIDADE DE SÃO PAULO

PARA

OBTENÇÃO DO GRAU DE MESTRE

EM

MATEMÁTICA APLICADA

Área de Concentração:

ANÁLISE NUMÉRICA

Orientador: Prof. Dr. Alexandre Megiorin Roma

Durante a elaboração deste trabalho o autor recebeu auxilio financeiro do CNPq.

São Paulo, 24 de Julho de 2000 


\title{
Método de Propagação de Ondas para Leis de Conservação com Termo Forçante
}

\author{
Este exemplar corresponde à redação \\ final da dissertação devidamente corrigida \\ e defendida por Hernán Arquímedes Cuti Gutiérrez \\ e aprovada pela comissão julgadora.
}

São Paulo, 24 de Julho de 2000.

Banca examinadora:

- Prof. Dr. Alexandre Megiorin Roma (orientador) - IME-USP

- Prof. Dr. Oswaldo Rio Branco de Oliveira - IME-USP

- Prof. Dr. Pedro Paulo Serpa Schirmer - IME-USP 
A Lupe e Fiorella, por todos estes anos de ausência.

A minha familia, pelo apoio durante todos estes anos. 


\section{Agradecimentos}

Meu agradecimento a todas as pessoas que ajudaram, direta ou indiretamente. na realizaçào deste trabalho.

- Agradeço em especial ao meu orientador Prof. Alexandre M. Roma, pela sugestão do tema, pelos inúmeros conselhos, pela sua paciência e por seu apoio durante todo este tempo.

- Ao Prof. Pedro Paulo Schirmer, pela sugestão de resolver numericamente leis de conservação adicionando-lhe termos forçantes e por ter me ajudado a resolver muitas dúvidas.

- Ao Prof. Luis Carlos de C. Santos, pela sugestão de empregar o CLAWPACK para resolver as leis de conservaçào com termo forçante.

- Ao Prof. Oswaldo Rio Branco de Oliveira, pelas sugestões durante la revisão do trabalho.

- Aos profesores do IME, pela sua contribuição na minha formação.

- Agradeço ao CNPq pelo auxílio financeiro.

- Aos funcionários do IME, pelo seu excelente trato e amabilidade, especialmente ao pessoal da CPG e da Biblioteca. 
- A mi esposa Lupe y a mi hija Fiore, por su comprensión durante todo este tiempo de aunsencia, y simplemente por ser como son.

- A mis dos grandes amigos de Trujillo, Ricardo Gutiérrez (mi "cumpa") y Manuel Montalvo (el "Pepo"), que siempre me recibieron bién.

- A los profesores de la Universidad nacional de Trujillo, por la contribuición en mi formación como matemático, especialmente a "Pepe" Olivencia por las muchas "conversas", a Obidio y Salomón por su aliento para hacer la Maestría, y a todos los colegas del Dpto. de Matemáticas.

- A mis amigos peruanos, que me trataron bien cuándo llegué a São Paulo, Walter, Elmer, Betty, Sonia, Beto, Carlos, Rósulo, Luis, Ariel, Gaspar, Luciano. No imaginan lo mucho que me ayudaron. También a los que fui conociendo durante mi permanencia en el IME, David, Nestor, Jorge, Omar, Rafael, Doris, Angela, Anita, Erick, Dora, Paco, Carlos, Jackie, Xyoby, Margot, Fredy Castelares, Katia, Delhi, Esteban, Julio, Martín, Roberto, Edwin, Fredy, Fernando, Rubén, María, Ingrid, etc.

- A mis dos grandes amigos, Santos y Nelson. Por las muchas "conversas" tomando café y almorzando los fines de semana, por los buenos y "malos" momentos y por haberme ayudado durante todo este tiempo. Y por soportar mis bromas a veces "pesadas".

- A Antonio (el "çhoque") por ser un buen amigo y por su chispa y a su esposa Josefa.

- A mis amigos brasileños, por su amistad, Walter, Edson, Calixto, Jocirei, Plácido, Ricardo, Uira, leandro, Rudimar, Daniela, Neusa, Olga, Alicia, Claudia, Albetan, Clezio, Rodrigo, Cibele, Samuel, Saraiva, Ezio, Mario, Ronaldo, Paulo.

- A mis amigos de los paises vecinos, Jorge, Irene, Cecilia, Raúl, Juan, Francisco, Mariano, Gonzalo, Iván, Claudia, Washington, Marcel, Karina, Bernardo, Domingo, Gladys, Hernán, Paola, Sandra, Jairo, Mauricio. Y a mi amigo africano Emmanuel. 


\section{Resumo}

Nesse trabalho, estudamos a teoria matemática envolvida nas leis de conservação hiperbólicas escalares, veremos como dados iniciais, embora sendo suaves, podem dar origem à formação de ondas de choque. Estudamos também o Método de Propagação de Ondas para a resolução das leis de conservação. Este método numérico é implementado no pacote computacional CLAWPACK, que é descrito nesse trabalho. Testamos nosso método numérico resolvendo leis de conservação com termo forçante, e propomos algumas conjecturas sobre este tipo de equações.

\section{Abstract}

In this work, we study the mathematical theory of the scalar hyperbolic conservation laws, we analyse how the initial conditions, even been smooth. can originate the formation of shock waves. We also study the Waves Propagation Method for the resolution of the conservation laws. This numeric method is implemented in the package computacional CLAWPACK, which is described in this work. We test our numeric method for solving such equations with source term, and we suggest some conjectures about these type of equations. 


\section{Índice}

$\begin{array}{ll}\text { Agradecimentos } & 4\end{array}$

$\begin{array}{ll}\text { Resumo } & 6\end{array}$

$\begin{array}{ll}\text { Lista de Figuras } & 8\end{array}$

$\begin{array}{ll}\text { Lista de Tabelas } & 10\end{array}$

1 Introdução 2

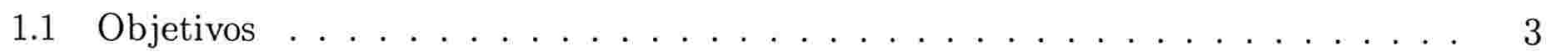

1.2 Revisão de Literatura . . . . . . . . . . . . . . . . . . . . . 4

1.3 Organização do trabalho . . . . . . . . . . . . . . . . . . . 6

2 Leis de Conservação Hiperbólicas $\quad 7$

2.1 Leis de Conservação . . . . . . . . . . . . . . . . . . . . . 8

2.2 A equação linear de adveç̧ão . . . . . . . . . . . . . . . . . . . . . . 11

2.3 Leis de conservação não lineares e ondas de choque . . . . . . . . . . . . . . . . . 12

2.4 Soluções Fracas . . . . . . . . . . . . . . . . . . . . . . . . . . . 15

2.5 A Equação de Burgers e o Problema de Riemman . . . . . . . . . . . . . . . . . . 18

2.6 Condições de Entropia . . . . . . . . . . . . . . . . . . . . . . . 23

3 Método de Propagação de Ondas $\quad 27$ 
3.1 Motivação: Sistemas Lineares . . . . . . . . . . . . . . . . . . . . . . . . 28

3.1.1 Correçōes de Segunda Ordem e Limitadores de Onda . . . . . . . . . . . . 33

3.2 Sistemas Não-Lineares . . . . . . . . . . . . . . . . . . . . . . . . . 35

3.3 O Caso Escalar . . . . . . . . . . . . . . . . . . . . . . . . . 40

4 O Software CLAWPACK $\quad 43$

4.1 Estrutura e organização do CLAWPACK . . . . . . . . . . . . . . . . 44

4.2 Tipo de equações resolvidas pelo CLAWPACK . . . . . . . . . . . . . . . 47

4.3 Condições de fronteira e Variáveis auxiliares . . . . . . . . . . . . . . . . 48

4.4 Termos forçantes . . . . . . . . . . . . . . . . . 50

5 Resultados Numéricos 53

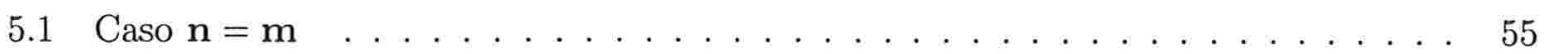

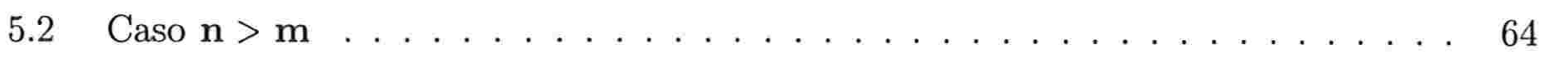

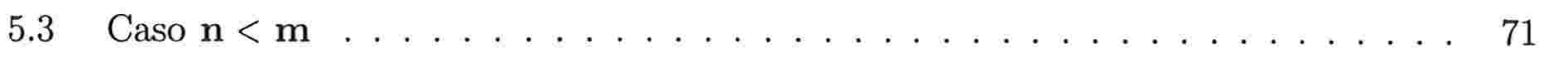

6 Conclusões $\quad 81$

$\begin{array}{ll}\text { A Apêndice A } & 83\end{array}$

A.1 O Método de Godunov . . . . . . . . . . . . . . . . . . . . . . . . . 83

$\begin{array}{ll}\text { B Apêndice B } & 87\end{array}$

B.1 CLAWPACK em duas dimensões . . . . . . . . . . . . . . . . . . . . . 87

B.1.1 Método de Godunov de Primeira ordem . . . . . . . . . . . . . . . . 88

B.1.2 Propagação transversal . . . . . . . . . . . . . . . . . . . . . . . . 89

B.1.3 Correções de segunda ordem e sua propagação transversal . . . . . . . . . 93

$\begin{array}{ll}\text { Índice Remissivo } & 97\end{array}$ 


\section{Lista de Figuras}

2.1 (a) Características $\left(q_{0}^{\prime}(x) \geq 0\right)$. (b) Intersecção de características $\left(q_{0}^{\prime}(x)<0, t=T_{b}\right) .14$

2.2 Formação de uma onda de choque. . . . . . . . . . . . . . . . 15

2.3 (a) Características da equação de Burgers com dado (2.27). Observe a intersecção delas para $t \geq 1$. (b) Formação da descontinuidade na equação de Burgers. . . . 19

2.4 Solução fraca da equação de Burgers com dado inicial (2.27) . . . . . . . . . . . 20

2.5 Características e choque na solução fraca. . . . . . . . . . . . . . . . . . . . 21

2.6 Características no plano da equação de Burgers para o caso II. . . . . . . . . . . 21

2.7 (a) Características no plano exibindo uma onda de rarefação. (b) Características no plano exibindo um leque de rarefação. . . . . . . . . . . . . . . . . 22

3.1 Construção da solução para o problema de Riemann em $(x, t) \ldots \ldots$. . . . . . . 30

3.2 Discretização do problema. . . . . . . . . . . . . . . . . 31

3.3 (a)-Dado inicial. (b),(c) e (d)-Solução numérica (com pontos) e exata (com linhas)

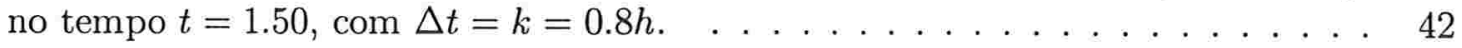

5.1 (a) Dado inicial (5.2), (b) Dado inicial (5.3), (c) Dado inicial (5.4), (d) Dado inicial (5.5), (e) Dado inicial (5.6). . . . . . . . . . . . . . . . . . . . 54

$5.2 m=n=2$. (a) Dado inicial (5.2). (c) Explosão da solução. . . . . . . . . . . . 57

$5.3 m=n=2$. (a) Dado inicial 5.3. (e) Explosão da solução. . . . . . . . . . . . . 57

$5.4 m=n=2$. (a) Dado inicial 5.4. (d)-(e) Formação do choque. (h) Explosão da solução. . . . . . . . . . . . . . . . . . . . . . . . . . . . 61

$5.5 m=n=2$. (a) Dado inicial 5.5. (d)-(e) Formação do choque. . . . . . . . . . 62 
$5.6 m=n=2$. (a) Dado inicial 5.6. (d)-(e) Formação do choque. (h) Explosão da solução. . . . . . . . . . . . . . . . . . . . . 63

$5.7 n=3, m=2$. (a)-Dado inicial (5.3). (b)-(e)-Continuação da solução sem a formação de choque. . . . . . . . . . . . . . . 67

$5.8 n=3, m=2$. (a)-Dado inicial (5.4). (c)-Formação do choque. . . . . . . . . . 68

$5.9 n=3, m=2$. (a)-Dado inicial (5.5). (d)-(e)-Formação do choque. . . . . . . . 69

$5.10 n=3, m=2$.(a)-Dado inicial (5.6). (b)-(c)-Formação do choque. . . . . . . . 70

$5.11 n=2$ e $m=4$. (a)-Dado inicial (5.3). (i)-Explosão da solução. . . . . . . . . . . 74

$5.12 n=2$ e $m=4$. (a)-Dado inicial (5.4). (d)-Explosão da solução. . . . . . . . . 75

$5.13 n=2$ e $m=4$. (a)-Dado inicial (5.5). (e)-(f)-Formação do choque. . . . . . . . 76

$5.14 n=2$ e $m=4$. (a)-Dado inicial (5.6). (e)-Explosão da solução. . . . . . . . . . 77

$5.15 n=2$ e $m=4$. (a)-Dado inicial (5.11). (b)-(e)-Formação do choque. . . . . . . 78

$5.16 n=2$ e $m=4$. (a)-Dado inicial (5.12). (f)-Explosão da solução. . . . . . . . . 79

A.1 Solução de problemas de Riemann independentes para dados constantes por partes 84

B.1 Discretização do problema. . . . . . . . . . . . . . . . 88

B.2 (a)-Propagação transversal na equação de advecção. (b)-As quatro diferenças de fluxos transversais para um sistema geral de equações. . . . . . . . . . . . . . . . 91 


\section{Lista de Tabelas}

5.1 Instantes de tempo e posições dos choques $\left(x_{c}, t_{c}\right)$ e/ou das explosões $\left(x_{e}, t_{e}\right) . \quad \ldots 55$

5.2 Instantes de tempo e posições dos choques $\left(x_{c}, t_{c}\right)$ e/ou das explosões $\left(x_{e}, t_{e}\right) . \ldots 55$

5.3 Instantes de tempo e posições dos choques $\left(x_{c}, t_{c}\right)$ e/ou das explosões $\left(x_{e}, t_{e}\right) . \ldots 56$

5.4 Instantes de tempo e posições dos choques $\left(x_{c}, t_{c}\right)$ e/ou das explosões $\left(x_{e}, t_{e}\right) . \ldots 56$

5.5 Instantes de tempo e posições dos choques $\left(x_{c}, t_{c}\right)$ e/ou das explosões $\left(x_{e}, t_{e}\right) . \ldots 64$

5.6 Instantes de tempo e posições dos choques $\left(x_{c}, t_{c}\right)$ e/ou das explosões $\left(x_{e}, t_{e}\right) . \quad$. 65

5.7 Instantes de tempo e posições dos choques $\left(x_{c}, t_{c}\right)$ e/ou das explosões $\left(x_{e}, t_{e}\right) . .65$

5.8 Instantes de tempo e posições dos choques $\left(x_{c}, t_{c}\right)$ e/ou das explosões $\left(x_{e}, t_{e}\right) . \ldots 72$

5.9 Instantes de tempo e posições dos choques $\left(x_{c}, t_{c}\right)$ e/ou das explosões $\left(x_{e}, t_{e}\right) . \quad$. 72 


\section{Introdução}

Nossas motivações para este trabalho foram o estudo e a resolução numérica de "leis de conservação" hiperbólicas escalares com termo forçante do tipo:

$$
\frac{\partial}{\partial t} q(x, t)+\frac{\partial}{\partial x} f(q(x, t))=\psi(q(x, t))
$$

para a qual damos a condição inicial (Problema de Cauchy),

$$
q(x, 0)=q_{0}(x) \quad, x \in \mathbb{R}
$$

O estudo destas equações é importante pois a modelagem matemática de muitos problemas em ciência e em engenharia conduz a equaçôes diferenciais parciais (EDPs) deste tipo. Como exemplos típicos podemos citar os problemas oriundos em aerodinâmica, em previsão meteorológica e em astrofísica.

Matematicamente, a propriedade mais interessante de tais equações é que elas admitem ondas de choque na sua solução, isto é, descontinuidades na solução ou em suas derivadas as quais podem se formar ainda que os dados iniciais sejam suaves.

Numericamente, quando tentamos calcular estas soluções, enfrentamos novos tipos de problemas. Por exemplo, se usarmos um método de baixa ordem (e.g., o de Godunov de primeira ordem) obtemos resultados que são muito "suaves" perto das descontinuidades cujo posicionamento e velocidade são muito imprecisos. Por outro lado se usarmos métodos de ordem mais alta (e.g., o de MacCormack ou o de Lax-Wendroff) aparecem oscilações não físicas na solução numérica. Estes problemas motivam a busca de métodos que produzam boas aproximações nas descontinuidades, isto é, métodos de alta resolução, como o Método de Propagação de Ondas 
que usaremos aqui.

Este capítulo está dividido em três seções. Na Seção 1.1, falaremos dos objetivos do trabalho, da idéia básica por trás do Método de Propagação de Ondas e de sua implementação no software $C L A W P A C K$. Na Seção 1.2, faremos uma revisão da literatura sobre a teoria de leis de conservação, sobre os métodos numéricos asociados e sobre a existência de outros pacotes para resolvê-las. Na última seção, Seção 1.3, falaremos sobre a organização do trabalho em seus diferentes capítulos.

\subsection{Objetivos}

Nossos objetivos com esse trabalho foram:

1. Estudar a teoria básica sobre leis de conservação hiperbólicas escalares;

2. Estudar o Método de Propagação de Ondas;

3. Aprender a baixar, instalar, modificar e, finalmente, empregar o CLAWPACK num problemateste particular, onde a função de fluxo $f$ e o termo forçante $\psi$ da equação (1.1) são dados respectivamente por $f(q)=\frac{1}{n} q^{n}, \psi(q)=q^{m}, m, n>1$, inteiros positivos.

O Método de Propagação de Ondas foi introduzido por LeVeque [19], [17], [20], [21]. O método está baseado na resolução de problemas de Riemann na interface entre duas células da malha e na aplicação de correções de segunda ordem e funções limitadoras às ondas resultantes, as quais são então propagadas.

LeVeque e colaboradores desenvolveram, no Departamento de Matemática Aplicada da Universidade de Washington, um software, o CLAWPACK (Conservation Laws Package). O CLAWPACK é um pacote de rotinas escritas em FORTRAN para resolver sistemas hiperbólicos de leis de conservação dependentes do tempo em uma, duas ou em três dimensões, usando o Método de Propagação de Ondas.

O CLAWPACK está livremente disponível na rede mundial de computadores e seu URL é: http://www . amath. washington.edu/ rjl/clawpack.html . 
Também pode ser encontrado em netlib. Para obtermos o índice de arquivos disponíveis enviar um e-mail para: netlib@research.att.com, com a seguinte mensagem

send pdes/claw/index

e para obtermos todos os arquivos, colocar a mensagem

send all from pdes/claw.

Alternativamente, podemos obter os arquivos via ftp na rede mundial de computadores no URL

ftp://netlib.bell-labs.com/netlib/pdes/claw/index.html.

Junto com o pacote está disponível também os manuais que descrevem como utilizá-lo, os algoritmos implementados e vários exemplos. Uma versão POSTSCRIPT destes manuais pode ser encontrada no diretório claw/doc do endereço acima.

\subsection{Revisão de Literatura}

Nesse trabalho, trataremos brevemente alguns dos aspectos relacionados ás equações hiperbólicas, apenas o necessário para entendermos o comportamento das leis de conservação. Abordagens mais completas podem ser encontradas, por exemplo, em John [11] ou em Kreiss-Lorenz [12], que trata este tema com ênfase nas equações de Navier-Stokes e nas equações de Euler.

A teoria matemática das leis de conservação data da década de 50 , com uma série de publicações feitas por Hopf [10], Oleinik [24] e Lax [13]. Entretanto, diversos trabalhos importantes já haviam sido feitos por pesquisadores da área de dinâmica dos fluidos. Uma excelente referência é o livro clássico de Courant-Friedrichs [8].

Lax [14] apresenta um pouco da teoria básica de leis de conservação não lineares. LeVeque [18] apresenta material suficiente para compreender a teoria das leis de conservação. O livro de EDPs de Renardy-Rogers [26] também apresenta um pouco sobre esta teoria. Já o livro de Smoller contém resultados relativamente recentes e uma teoria mais completa que os anteriores, sendo uma excelente referência. 
Com relação aos métodos numéricos, os métodos de alta resolução baseados na resolução de problemas de Riemann, foram propostos primeiramente para uma certa classe de problemas onde as descontinuidades no problema ou na sua solução levavam a certas dificuldades com os métodos padrões de diferenças finitas. Em particular, a idéia básica para obtermos métodos de alta resolução baseados em alguma forma de limitadores de inclinação ou limitadores de fluxo tem uma longa história. Detalhes destes métodos podem ser encontrados, por exemplo, em Sweby [30] e em Goodman-LeVeque [9]. Muitas outras referências podem ser encontradas no livro de LeVeque [18].

Quanto ao Método de Propagação de Ondas, ele foi desenvolvido para resolver sistemas hiperbólicos multi-dimensionais de EDPs. Ủma das vantagens do método é que ele permite extensões para equações não conservativas, e para problemas que incluem uma função capacidade. O método pode ser estendido para duas ou três dimensões por intermédio de uma aproximação natural da propagação de ondas, a qual captura os termos de derivadas cruzadas necessários para a precisão de segunda ordem. Detalhes podem ser encontrados em LeVeque [21]. No contexto de advecção, uma visão geral dos métodos relacionados é dado por LeVeque [20].

O Método de Propagação de Ondas foi implementado por LeVeque e colaboradores na Universidade de Washington, no software CLAWPACK, que é uma coleção de rotinas em FORTRAN. O pacote inclui muitos exemplos para uma variedade de sistemas hiperbólicos.

O CLAWPACK tem sido combinado com o código de refinamento adaptativo de malhas de Berger [1], [2], [3], [4], [6]. Como resultado, obteve-se um pacote de refinamento adaptativo muito geral, com todas as características do Método de Propagação de Ondas. Os detalhes da implementação do pacote podem ser encontrados em Berger-LeVeque [5]. O software AMRCLAW (Adaptive Mesh Refinement of Conservation Laws) está disponível na rede mundial de computadores e seu URL é:

http://www . amath. washington.edu/ ${ }^{\sim}$ rjl/amrclaw/ . 


\subsection{Organização do trabalho}

No Capítulo 2, trataremos da teoria matemática envolvida nas leis de conservação, inclusive o motivo pelo qual os dados iniciais, ainda que suaves, dão origem à formação de ondas de choque. Discutimos nesse contexto a necessidade de ampliar o espaço de soluções clássicas, dando origem às soluções fracas, e seu impacto sobre a unicidade das soluções. Esse capítulo termina enunciando um teorema sobre existência e unicidade.

No Capítulo 3, trataremos do método utilizado para resolver numericamente as leis de conservação hiperbólicas escalares, o Método de Propagação de Ondas. No início ele será de primeira ordem, pois é uma reformulação do método de Godunov na forma de propagação de ondas. Mas, depois de agregar-lhe termos de correção de segunda ordem e empregar funções limitadoras, nós o transformaremos num método de alta resolução.

No Capítulo 4, explicitaremos tudo o que se refere ao software CLAWPACK, o qual utiliza o Método de Propagação de Ondas para resolver as leis de conservação hiperbólicas. Comentaremos os algoritmos empregados e os vários exemplos usados no trabalho.

No Capítulo 5, mostraremos os diferentes resultados numéricos obtidos com a utilização do CLAWPACK, para diferentes valores de $m$ e $n$ em $f(q)=\frac{1}{n} q^{n}$ e em $\psi(q)=q^{m}$. Os resultados foram agrupados em três casos: $m=n, n<m$ e $n>m$.

Acreditamos que, com este trabalho, nossas principais aquisições e contribuições sejam:

- O estudo do Método de Propagação de Ondas como um método geral para resolver leis de conservação hiperbólicas escalares.

- A divulgação e utilização do pacote CLAWPACK, assim como onde encontrá-lo e como instalá-lo. 


\section{Leis de Conservação Hiperbólicas}

Neste capítulo, estudaremos a teoria das leis de conservação hiperbólicas escalares.

Na Seção 2.1, introduziremos as formas integrais e diferencial das leis de conservação. As primeiras são muito importantes pois quando falarmos de soluções fracas, o ponto de partida deverá ser a formulação integral. A seguir, introduziremos a forma diferencial, a qual pode ser obtida assumindo diferenciabilidade da solução. Finalizaremos esta seção introduzindo termos forçantes à lei de conservação.

Na Seção 2.2, estudaremos um caso simples de lei de conservação: a equação de advecção linear. Isto motivará a definição de solução da lei de conservação no caso em que os dados iniciais não sejam suaves.

Na Seção 2.3, analisaremos o caso não linear obtendo a solução implicitamente. A seguir, estudaremos a formação de ondas de choque e veremos a necessidade de estendermos o conceito de solução clássica. Mostraremos esta extensão na Seção 2.4 definindo as soluções fracas, incluindo também condições necessárias, a Condição de Rankine-Hugoniot.

Na Seção 2.5, falaremos da equação de Burgers, a qual irá nos servir de modelo para testar o método numérico. Também falaremos do problema de Riemann, resolvendo-o para a equação de Burgers. Veremos aqui a perda de unicidade da solução, problema que será resolvido na última seção, a Seção 2.6, com o auxilio das Condições de Entropia. 


\subsection{Leis de Conservação}

Em geral, as leis de conservação são sistemas de equações diferenciais parciais não lineares dependentes do tempo, com uma estrutura particularmente simples. Em uma dimensão as equações tomam a forma

$$
\frac{\partial}{\partial t} q(x, t)+\frac{\partial}{\partial x} f(q(x, t))=0
$$

onde $q: \mathbb{R} \times \mathbb{R}^{+} \longrightarrow \mathbb{R}^{m}$ é um vetor m-dimensional de quantidades conservadas, ou variáveis de estado, tais como massa, momento e energia. A função vetorial $f: \mathbb{R}^{m} \longrightarrow \mathbb{R}^{m}$, ao menos de classe $C^{2}$, é chamada função de fluxo para o sistema de leis de conservação.

Observe que $q_{j}$ é a função densidade para a j-ésima variável de estado, com a interpretação que $\int_{x_{1}}^{x_{2}} q_{j}(x, t) d x$ é a quantidade total desta variável de estado no intervalo $\left[x_{1}, x_{2}\right]$ no tempo $t$.

Assumiremos que o sistema (2.1) é hiperbólico, ou seja, que a matriz Jacobiana $f^{\prime}(q), m \times m$, é diagonalizável tendo apenas autovalores reais.

Num espaço de duas dimensões, o sistema de leis de conservação assume a forma

$$
\frac{\partial}{\partial t} q(x, y, t)+\frac{\partial}{\partial x} f(q(x, y, t))+\frac{\partial}{\partial y} g(q(x, y, t))=0,
$$

onde $q: \mathbb{R}^{2} \times \mathbb{R}^{+} \longrightarrow \mathbb{R}^{m}$ e $f, g: \mathbb{R}^{m} \longrightarrow \mathbb{R}^{m}$ são as funções de fluxo. A generalização para mais dimensões é imediata. Hiperbolicidade significa aqui que qualquer combinação linear $\alpha f^{\prime}(q)+\beta g^{\prime}(q), \alpha, \beta \in \mathbb{R}$, dos Jacobianos das funções de fluxo seja diagonalizável com autovalores reais.

Como já mencionamos anteriormente, a título de simplificação, abordaremos apenas problemas escalares com uma variável de estado $(m=1)$.

Tipicamente as funções de fluxo são funções não-lineares de $q$. Em geral não conseguimos exibir soluções exatas para estas equações e, portanto, a necessidade de empregar métodos 
numéricos para sua solução.

Veremos a seguir as formas integrais e diferencial das leis de conservação escalares.

A primeira forma integral é dada por:

$$
\frac{d}{d t} \int_{x_{1}}^{x_{2}} q(x, t) d x=f\left(q\left(x_{1}, t\right)\right)-f\left(q\left(x_{2}, t\right)\right)
$$

Obtemos uma segunda forma integral integrando (2.3) no tempo, de $t_{1}$ até $t_{2}$, com $t_{2}>t_{1}$ :

$$
\int_{x_{1}}^{x_{2}} q\left(x, t_{2}\right) d x=\int_{x_{1}}^{x_{2}} q\left(x, t_{1}\right) d x+\int_{t_{1}}^{t_{2}} f\left(q\left(x_{1}, t\right)\right) d t-\int_{t_{1}}^{t_{2}} f\left(q\left(x_{2}, t\right)\right) d t .
$$

Se $f$ e $q$ forem diferenciáveis, poderemos obter, usando

$$
q\left(x, t_{2}\right)-q\left(x, t_{1}\right)=\int_{t_{1}}^{t_{2}} \frac{\partial}{\partial t} q(x, t) d t
$$

$\mathrm{e}$

$$
f\left(q\left(x_{2}, t\right)\right)-f\left(q\left(x_{1}, t\right)\right)=\int_{x_{1}}^{x_{2}} \frac{\partial}{\partial x} f(q(x, t)) d x,
$$

a forma diferencial da lei de conservação

$$
\frac{\partial}{\partial t} q(x, t)+\frac{\partial}{\partial x} f(q(x, t))=0 .
$$

As formas integrais admitem uma interpretação física, como por exemplo a conservação de massa em um problema de dinâmica de gases unidimensional. Para isto, suponha que tenhamos o fluxo de um gás em um tubo, cujas propriedades, tais como densidade e velocidade, são assumidas como sendo constantes através de cada seção transversal. Seja $x$ a distância ao longo do tubo e $\rho(x, t)$ a densidade de massa do gás no ponto $x$ e no instante de tempo $t$. A densidade é definida de tal forma que a massa total do gás, em qualquer seção de $x_{1}$ a $x_{2}$, seja dada pela integral da densidade

$$
\left\{\text { massa total em }\left[x_{1}, x_{2}\right] \text { no instante } t\right\}=\int_{x_{1}}^{x_{2}} \rho(x, t) d x .
$$

Assumindo que as paredes do tubo sejam impermeáveis e que a massa não possa ser criada nem destruída, então a massa nesta seção pode variar somente se o gás está fluindo através dos pontos da fronteira $x_{1}$ e $x_{2}$. 
Agora seja $v(x, t)$ a velocidade do gás no ponto $x$ e tempo $t$. Então o fluxo de massa passando por este ponto é dado por

$$
\{\text { fluxo de massa em }(x, t)\}=\rho(x, t) v(x, t) \text {. }
$$

Assim sendo, a taxa de variação da massa em $\left[x_{1}, x_{2}\right]$ é dado pela diferença dos fluxos de massa em $x_{1}$ e $x_{2}$,

$$
\frac{d}{d t} \int_{x_{1}}^{x_{2}} \rho(x, t) d x=\rho\left(x_{1}, t\right) v\left(x_{1}, t\right)-\rho\left(x_{2}, t\right) v\left(x_{2}, t\right) .
$$

Como podemos ver, esta é a primeira forma integral da lei de conservação da massa. A segunda forma integral é obtida integrando no tempo a equação (2.7) de $t_{1}$ até $t_{2}$, obtemos assim uma expressão para a massa em $\left[x_{1}, x_{2}\right]$ no tempo $t_{2}>t_{1}$ em termos da massa no tempo $t_{1}$ e do fluxo total em cada fronteira durante este período de tempo:

$$
\int_{x_{1}}^{x_{2}} \rho\left(x, t_{2}\right) d x=\int_{x_{1}}^{x_{2}} \rho\left(x, t_{1}\right) d x+\int_{t_{1}}^{t_{2}} \rho\left(x_{1}, t\right) v\left(x_{1}, t\right) d t-\int_{t_{1}}^{t_{2}} \rho\left(x_{2}, t\right) v\left(x_{2}, t\right) d t .
$$

Equivalentemente, como fizemos para o caso geral acima, supondo que $\rho(x, t)$ e $v(x, t)$ sejam diferenciáveis, obtemos a forma diferencial da lei de conservação de massa

$$
\rho_{t}+(\rho v)_{x}=0
$$

As formas integrais (2.3) e (2.4) correspondem a leis de conservação físicas fundamentais. É importante observarmos que estas equações têm sentido mesmo que a função $q(x, t)$ seja descontínua! Por outro lado, sua versão diferencial (2.5) só será válida quando $q(x, t)$ for suave.

Mais detalhes podem ser encontrados em LeVeque [18] e para uma interpretação física mais geral ver Lax [14].

Para vermos como termos forçantes podem ser incorporados à lei de conservação, reconsideremos a expressão (2.3) na situação onde existe outra fonte afetando a quantidade conservada $q$ no intervalo $\left[x_{1}, x_{2}\right], \int_{x_{1}}^{x_{2}} q(x, t) d x$. Denotaremos esta fonte por $\psi(q)$. Desta forma $\psi(q(x, t)$ representará o incremento em $q$ (ou decrescimento, se $\psi<0$ ) no ponto $(x, t)$ por unidade de tempo. Então no lugar de (2.3) teremos

$$
\frac{d}{d t} \int_{x_{1}}^{x_{2}} q(x, t) d x=f\left(q\left(x_{1}, t\right)\right)-f\left(q\left(x_{2}, t\right)\right)+\int_{x_{1}}^{x_{2}} \psi(q(x, t)) d x .
$$


Procedendo como antes, assumindo que $q, f, e \psi$ são suaves, obteremos a lei de conservação não homogênea

$$
q_{t}+f(q)_{x}=\psi(q)
$$

\subsection{A equação linear de advecção}

Antes de começarmos o estudo do caso não linear, veremos primeiro um caso simples de lei de conservação, a equação linear de advecção ,

$$
q_{t}+u q_{x}=0,
$$

onde $u=$ constante é a velocidade de adveç̧ão, para a qual será dada uma condição inicial (Problema de Cauchy), $q(x, 0)=q_{0}(x)$.

Curvas características são curvas satisfazendo a equação diferencial ordinária $x^{\prime}(t)=u$, $x(0)=x_{0}$, isto é, são curvas da forma $x-u t=x_{0}$.

Observe que a solução de (2.13), $q(x, t)$, é constante ao longo destas curvas características. De fato,

$$
\begin{aligned}
\frac{d}{d t} q(x(t), t) & =\frac{\partial}{\partial t} q(x(t), t)+\frac{\partial}{\partial x} q(x(t), t) x^{\prime}(t) \\
& =q_{t}+u q_{x} \\
& =0,
\end{aligned}
$$

donde $q(x(t), t)=$ constante. Assim $q(x, t)=q\left(x_{0}, 0\right)=q_{0}\left(x_{0}\right)$. Portanto, a solução é dada por

$$
q(x, t)=q_{0}(x-u t)
$$

para $t \geq 0$. Isto quer dizer que quando o tempo evolui, o dado inicial simplesmente propaga-se invariavelmente para a direita (se $u>0$ ) ou para a esquerda (se $u<0$ ) com velocidade $u$.

O conceito de curva característica pode ser enunciado de uma forma mais geral. Para isto consideremos o seguinte problema de Cauchy:

$$
\left\{\begin{array}{l}
a(x, t, q) q_{t}+b(x, t, q) q_{x}=c(x, t, q) \\
q(\gamma(s))=q_{0}(s)
\end{array}\right.
$$


onde $\gamma$ é uma curva parametrizada por

$$
x=f(s), \quad y=g(s), \quad z=h(s) .
$$

A superfície solução $z=q(x, t)$ é chamada de superfície integral da EDP (2.15).

As curvas que satisfazem o sistema de equações diferenciais ordinárias

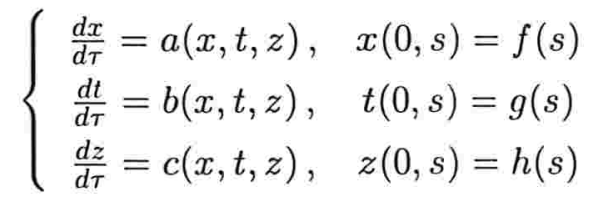

são chamadas de curvas características. Para mais detalhes ver John [11].

Voltando à equação linear de advecção vimos que a solução ao longo de uma curva característica (2.14) depende somente do valor $q_{0}\left(x_{0}\right)$. A derivada em $x$ não foi requerida para a construção de $q(x, t)$ a partir de $q_{0}(x)$. Então podemos definir uma "solução" para a EDP ainda que o dado inicial $q_{0}(x)$ não seja uma função suave. Observemos que se $q_{0}(x)$ tiver uma singularidade em algum ponto $x_{0}$ (uma descontinuidade em $q_{0}$ ou em alguma derivada), então a solução $q(x, t)$ terá uma singularidade da mesma ordem ao longo da curva características através de $x_{0}$. Esta é uma propriedade fundamental de equaçōes hiperbólicas lineares : singularidades propagam-se somente ao longo de características.

Se o dado inicial $q_{0}$ não for derivável em algum ponto, então $q(x, t)$ já não é mais uma solução clássica da equação diferencial. Entretanto, esta função satisfará a forma integral da lei de conservação, a qual continua válida para funções de $q$ não suaves.

\subsection{Leis de conservação não lineares e ondas de choque}

Consideremos agora leis de conservação não lineares

$$
q_{t}+f(q)_{x}=0
$$

com a condição inicial

$$
q(x, 0)=q_{0}(x)
$$


onde $f(q)$ é uma função não linear de $q$. Assumiremos, salvo menção feita em contrário, que $f(q)$ é uma função convexa, isto é, $f^{\prime \prime}(q)>0$ para todo $q$.

Podemos escrever (2.17) na forma

$$
q_{t}+f^{\prime}(q) q_{x}=0
$$

forma análoga a da equação de advecção linear (2.13). Neste caso também, a solução $q(x, t)$ permanece constante ao longo das curvas características, obtidas quando resolvemos a equação diferencial ordinária

$$
\left\{\begin{array}{l}
x^{\prime}(t)=f^{\prime}(q(x, t)), \\
x(0)=x_{0} .
\end{array}\right.
$$

De fato,

$$
\begin{aligned}
\frac{d}{d t} q(x(t), t) & =\frac{\partial}{\partial t} q(x(t), t)+\frac{\partial}{\partial x} q(x(t), t) x^{\prime}(t) \\
& =q_{t}+f^{\prime}(q) q_{x} \\
& =0 .
\end{aligned}
$$

Assim sendo, vemos que, como $q(x(t), t)$ é constante, $f(q(x(t), t))$ é constante e portanto a tangente $x^{\prime}(t)=f(q(x(t), t))$ é constante. Isto significa que as curvas características são linhas retas!

Se supusermos que o dado inicial (2.18) é suave, podemos usar este fato para determinarmos a solução $q(x, t)$. Sobre as características

$$
x=x_{0}+t f^{\prime}\left(q_{0}\left(x_{0}\right)\right), q(x(t), t)=\text { constante }=q_{0}\left(x_{0}\right) .
$$

Dessa forma, obtemos a equação implícita

$$
q(x, t)=q_{0}\left(x-t f^{\prime}(q)\right)
$$

No que segue, iremos provar que a solução (2.21) desenvolve ondas de choque, isto é. a solução desenvolve descontinuidades mesmo que os dados iniciais sejam suaves.

Supondo que a condição inicial $q_{0}(x)$ é diferenciável, podemos aplicar o Teorema da Função Implícita à equação (2.21), para obter $q$, para $t$ suficientemente pequeno, como uma função diferenciável de $x$ e $t$ somente. 
Seja $F(x, t, q)=q-q_{0}\left(x-t f^{\prime}(q)\right)$, uma função de variáveis $x, t$ e $q$. Então temos que

$$
F_{x}=-q_{0}^{\prime}, \quad F_{t}=q_{0}^{\prime} f^{\prime}(q), \quad F_{q}=1+t q_{0}^{\prime} f^{\prime \prime}(q)
$$

Agora para $t=0$, temos que $F_{q}=1 \neq 0$, por tanto pelo Teorema da Função Implícita podemos obter $q$ em função de $x$ e $t$ ao redor de $t=0$. Além disso suas derivadas parciais serão

$$
\begin{gathered}
q_{t}=-\frac{F_{t}}{F_{q}}=-\frac{q_{0}^{\prime} f^{\prime}(q)}{1+t q_{0}^{\prime} f^{\prime \prime}(q)}, \\
q_{x}=-\frac{F_{x}}{F_{q}}=\frac{q_{0}^{\prime}}{1+t q_{0}^{\prime} f^{\prime \prime}(q)} .
\end{gathered}
$$

Se $q_{0}^{\prime} \geq 0$ para todo $x$, então $q_{t}$ e $q_{x}$ ficam limitadas para todo $t>0$. Mais ainda, $q_{0}(x)$ será uma função crescente de $x$, isto quer dizer que as características saindo do eixo- $x$ divergem na direção positiva de $t$, isto é, as características não se intersectam, simplesmente cobrem o semiplano $t>0$, (veja Figura 2.1-(a)).

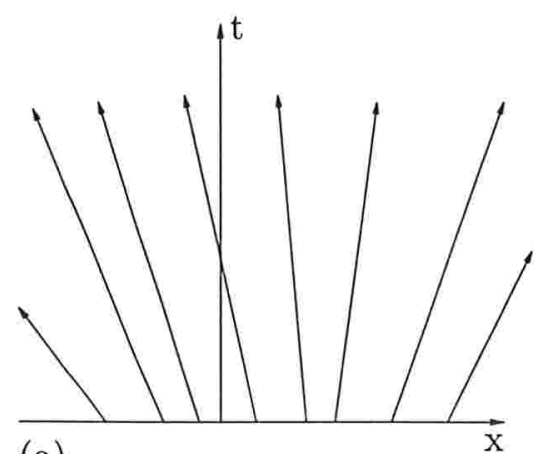

(a)

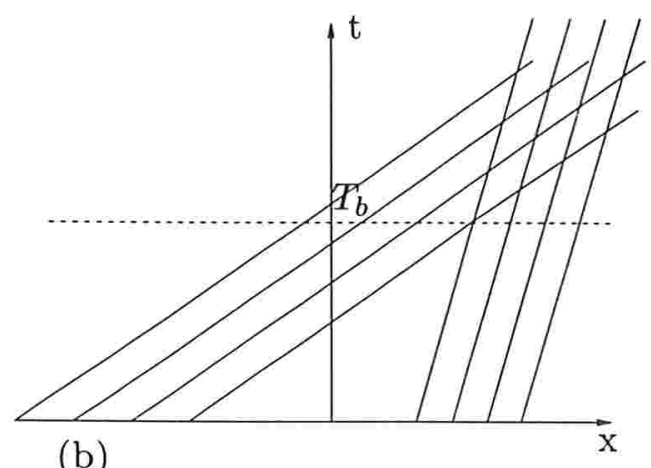

(b)

Figura 2.1: (a) Características $\left(q_{0}^{\prime}(x) \geq 0\right)$. (b) Interseç̧ão de características $\left(q_{0}^{\prime}(x)<0, t=T_{b}\right)$.

No caso quando $q_{0}^{\prime}(x)<0\left(q_{x}(x, 0)<0\right)$ em algum ponto, ambos $q_{t}$ e $q_{x}$ tenderão ao infinito quando $1+t q_{0}^{\prime} f^{\prime \prime}(q)$ tende a zero. Isto quer dizer que para algum instante de tempo $t$ a equação (2.21) não terá uma única solução. Isto acontece quando as curvas características se intersectan, o que é conseqüência de se ter $q_{0}^{\prime}(x)<0$ (veja Figura 2.1-(b)).

Se igualarmos a zero o denominador de $q_{x}$, isto é, $1+t q_{0}^{\prime} f^{\prime \prime}(q)=0$, temos que $t=$ $-1 / f^{\prime \prime}(q) q_{0}^{\prime}(x)$. Então o tempo onde as características se intersectan pela primeira vez será $t=T_{b}=-1 / f^{\prime \prime}(q) \min _{x}\left\{q_{0}^{\prime}(x)\right\}$, neste instante de tempo a onda se quebra e temos a formação 
de choques. A partir deste instante não existirá mais solução clássica da EDP (veja Figura 2.2). Em muitos problemas da física, equações do tipo (2.17) aparecem de modo natural, e as soluçōes descontínuas têm sido consideradas com muito sucesso. Na seção a seguir mostraremos como superar este problema.

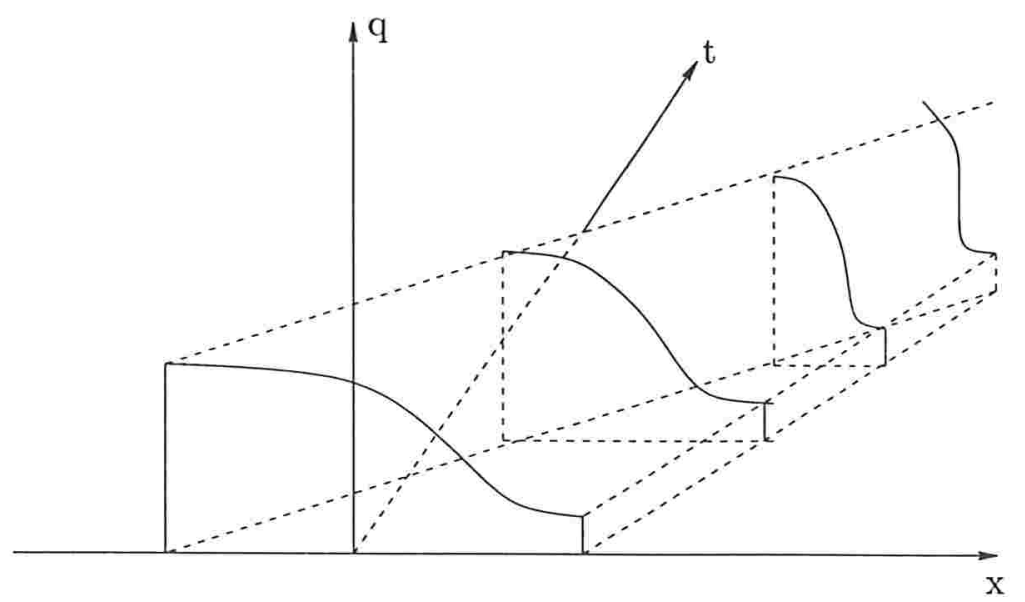

Figura 2.2: Formação de uma onda de choque.

\subsection{Soluções Fracas}

Uma maneira de definirmos soluções generalizadas para a equação (2.17), isto é, soluções que não requerem diferenciabilidade, é voltarmos à forma integral da lei de conservação e dizer que $q(x, t)$ é uma solução generalizada se (2.4) for satisfeita para todo $x_{1}, x_{2}, t_{1}, t_{2}$ (veja Lax [14]).

Existe uma outra definição equivalente que resulta numa formulação integral diferente, mais conveniente para trabalharmos. A idéia básica é tomarmos a EDP, multiplicá-la por uma função teste suave, integrá-la uma ou mais vezes sobre algum domínio e, então, usar integração por partes para remover as derivadas da função $q$, transferindo-as para a função teste suave. O resultado é uma formulação envolvendo poucas derivadas sobre $q$, requerendo portanto menos suavidade. Em nosso caso usaremos funções testes $\phi \in C_{0}^{1}\left(\mathbb{R} \times \mathbb{R}^{+}\right)$, onde $C_{0}^{1}$ é o espaço de funções que são continuamente diferenciáveis com suporte compacto. 
Multiplicando $q_{t}+f(q)_{x}=0$ por $\phi(x, t)$ e integrando no espaço e no tempo teremos,

$$
\int_{0}^{\infty} \int_{-\infty}^{+\infty}\left[\phi q_{t}+\phi f(q)_{x}\right] d x d t=0
$$

Agora, integrando por partes, obtemos

$$
\int_{0}^{\infty} \int_{-\infty}^{+\infty}\left[\phi_{t} q+\phi_{x} f(q)\right] d x d t+\int_{-\infty}^{+\infty} \phi(x, 0) q(x, 0) d x=0
$$

Definição 2.4.1 A função $q(x, t)$ é chamada uma solução fraca da lei de conservação se (2.22) for verificado para toda função teste $\phi \in C_{0}^{1}\left(\mathbb{R} \times \mathbb{R}^{+}\right)$.

Com esta definição, ampliamos bastante a classe de funções que podem ser soluções do problema de valor inicial (2.17), (2.18). Em particular, toda solução clássica $q \in C^{1}\left(\mathbb{R} \times \mathbb{R}^{+}\right)$, é uma solução fraca. Embora uma solução fraca possa não ser diferenciável, ela deve ser uma solução global. Não há sentido falarmos de solução fraca local, embora tenhamos que falar de solução clássica local. Outro detalhe a ser observado é que o conceito de solução fraca é o mais natural. Se nos reportarmos à formulação integral das leis de conservação (2.4), vemos que para que estas equações sejam satisfeitas não há necessidade de que as funções envolvidas sejam diferenciáveis. O conceito de solução fraca está intimamente ligado à teoria de distribuições.

Agora que já definimos uma solução fraca, no teorema a seguir daremos condições necessárias para que uma solução fraca possa ser descontínua.

\section{Teorema 2.4.1 (Condição de Rankine-Hugoniot)}

Seja $\Omega$ uma vizinhança aberta no semiplano superior aberto e suponhamos que uma curva $\Gamma$ : $(\alpha, \beta) \ni t \longmapsto x(t)$ divide $\Omega$ em duas partes $\Omega_{e} e \Omega_{d}$, ambas a esquerda e direita da curva, respectivamente. Seja $q$ uma solução fraca da equação (2.17) (as condições iniciais não são de interesse aqui) tal que:

1. q é uma solução clássica de (2.17) em $\Omega_{e}$ e $\Omega_{d}$,

2. q tem uma descontinuidade de salto $[q]$ na curva $\Gamma$, a onda de choque,

3. $O$ salto $[q]$ é contínuo ao longo de $\Gamma, e$ 
para qualquer $p \in \Gamma$, seja $\mathrm{s}:=x^{\prime}(p)$ a tangente de $\Gamma$ em $p$, a velocidade da onda de choque. Então a seguinte relação verifica-se entre a curva e os saltos,

$$
\mathbf{s}[q]=[f(q)] .
$$

Aqui, para qualquer $p=(\bar{x}, \bar{t}) \in \Gamma$ definimos :

$$
[q](p):=q_{d}(p)-q_{e}(p):=\lim _{\left(x_{d}, t_{d}\right) \stackrel{d}{\rightarrow} p} q\left(x_{d}, t_{d}\right)-\lim _{\left(x_{e}, t_{e}\right) \stackrel{e}{\rightarrow} p} q\left(x_{e}, t_{e}\right)
$$

O teorema anterior nos diz como é que a onda de choque se propaga ao longo do tempo, fornecendo-nos condições suficientes para que calculemos a velocidade de propagação da onda de choque. Este resultado será usado para resolver os problemas de Riemann, na proxima seção. Para a prova deste teorema ver o livro de Renardy e Rogers [26].

Devemos tomar cuidado ao manipularmos algebricamente leis de conservação. A transformação de uma forma diferencial numa outra pode alterar a estrutura do espaço de soluções fracas. Por exemplo, a equação de Burgers,

$$
q_{t}+\left(\frac{1}{2} q^{2}\right)_{x}=0
$$

é equivalente à equação

$$
\left(q^{2}\right)_{t}+\left(\frac{2}{3} q^{3}\right)_{x}=0
$$

a qual é novamente uma lei de conservação, só que agora para $q^{2}$, com função de fluxo $f\left(q^{2}\right)=$ $\frac{2}{3}\left(q^{2}\right)^{3 / 2}$. As equações (2.24) e (2.25) têm a mesma solução suave $q(x, t)=q_{0}(x-t q)$. Porém, elas têm diferentes soluções fracas. Por exemplo, se considerarmos a condição inicial

$$
q_{0}(x)=\left\{\begin{array}{lll}
1, & \text { se } & x \leq 0 \\
0, & \text { se } & x \geq 0
\end{array}\right.
$$

teremos como solução fraca de (2.24)

$$
q(x, t)= \begin{cases}1, & \text { se } \quad x \leq \frac{t}{2} \\ 0, & \text { se } \quad x>\frac{t}{2}\end{cases}
$$

pois vemos que, de acordo com o teorema anterior, (1) q é solução clássica de (2.24) em ambos lados da curva de descontinuidade $\Gamma: x(t)=t / 2 ;(2) q$ tem uma descontinuidade de salto 
$[q]=q_{e}-q_{d}=1$, e obviamente (3) o salto $q$ é contínuo ao longo de $\Gamma$. Portanto, a velocidade do choque é

$$
s_{1}=\frac{[f(q)]}{[q]}=\frac{1}{2} .
$$

No entanto, a única solução fraca para (2.25) é dada por

$$
q(x, t)= \begin{cases}1, & \text { se } x \leq \frac{2 t}{3}, \\ 0, & \text { se } x>\frac{2 t}{3},\end{cases}
$$

para a qual a curva de descontinuidade $\Gamma$ é dada por $x(t)=2 t / 3$, ao longo da qual ela satisfaz as condições do teorema anterior. Portanto, temos que a velocidade do choque será

$$
s_{2}=\frac{[f(q)}{[q]}=\frac{2}{3} .
$$

Das considerações anteriores, vemos que $s_{1} \neq s_{2}$, e as duas equações têm diferentes soluções fracas. A dedução de (2.25) e de (2.24) requer a manipulação de derivadas de uma maneira que somente é válida quando $q$ é suave.

\subsection{A Equação de Burgers e o Problema de Riemman}

Agora trataremos o problema mais famoso no campo das leis de conservação, a equação de Burgers ${ }^{1}$, para a qual a função de fluxo é dada por $f(q)=\frac{1}{2} q^{2}$, e portanto (2.17) toma a forma

$$
q_{t}+q q_{x}=0
$$

A seguir, resolveremos a equação de Burgers para dados iniciais não suaves, como por exemplo:

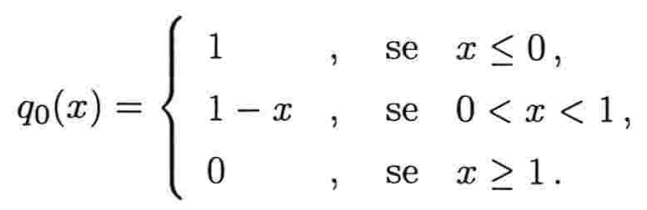

O gráfico das curvas características neste caso aparecem na Figura 2.3-(a).

Vemos que as características que partem de $x=0$ e $x=1$ se encontram em $t=1$, que é o tempo onde a onda começa a "quebrar". A partir de $t=1$, temos uma descontinuidade, uma

\footnotetext{
${ }^{1} \mathrm{Na}$ verdade, deve ser chamada equação de Burgers não-viscosa, pois a equação originalmente estudada por Burgers, inclui um termo viscoso, isto é, $q_{t}+q q_{x}=\mu q_{x x}$.
} 


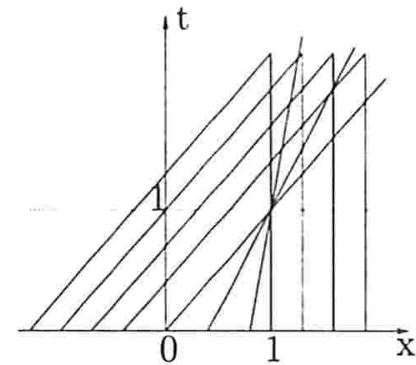

(a)

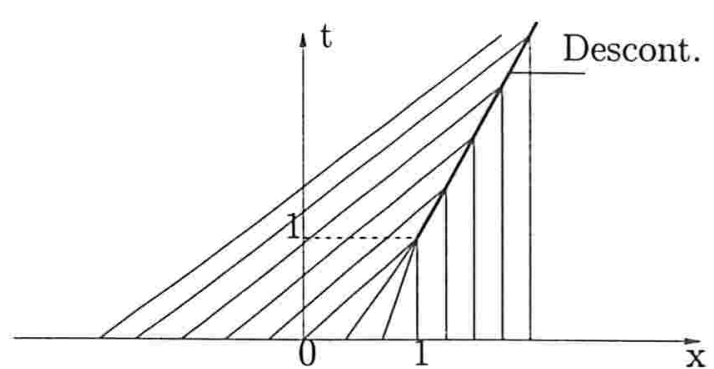

(b)

Figura 2.3: (a) Características da equação de Burgers com dado (2.27). Observe a intersecção delas para $t \geq 1$. (b) Formação da descontinuidade na equação de Burgers.

onda de choque, que separa o estado esquerdo, $q_{e}=1$, do estado direito, $q_{d}=0$. A velocidade de propagação do choque foi calculada de acordo com a condição de salto (2.23), com $f(q)=\frac{1}{2} q^{2}$, sendo $s=1 / 2$. Uma vez introduzida a descontinuidade, as características no plano $x-t$ ficam como na Figura 2.3-(b). Portanto, a solução fraca deste exemplo, para todo $t$, é dada por

$$
\begin{gathered}
q(x, t)=\left\{\begin{array}{lll}
1 & , & \text { se } x \leq t \quad \text { e } t<1, \\
(1-x) /(1-t) & , & \text { se } t<x<1 \quad \text { e } t<1, \\
0 & , & \text { se } x \geq 1 \quad \text { e } t<1,
\end{array}\right. \\
q(x, t)=\left\{\begin{array}{llll}
1, & \text { se } x<1+\frac{1}{2}(t-1) & \text { e } t \geq 1, \\
0, & \text { se } x>1+\frac{1}{2}(t-1) & \text { e } t \geq 1 .
\end{array}\right.
\end{gathered}
$$

Observe que a solução (2.28), para $t<1$, é solução clássica. Na Figura 2.4. temos o gráfico das ondas no espaço.

A lei de conservação (2.26) com dados iniciais constante por partes da forma

$$
q(x, 0)= \begin{cases}q_{e}, & \text { se } x<0 \\ q_{d}, & \text { se } x \geq 0\end{cases}
$$

é conhecido como problema de Riemann.

O estudo do problema de Riemann é pedagogicamente importante, pois permite-nos analisar uma variedade de ondas de comportamento parecido com choques. O problema de Riemann 


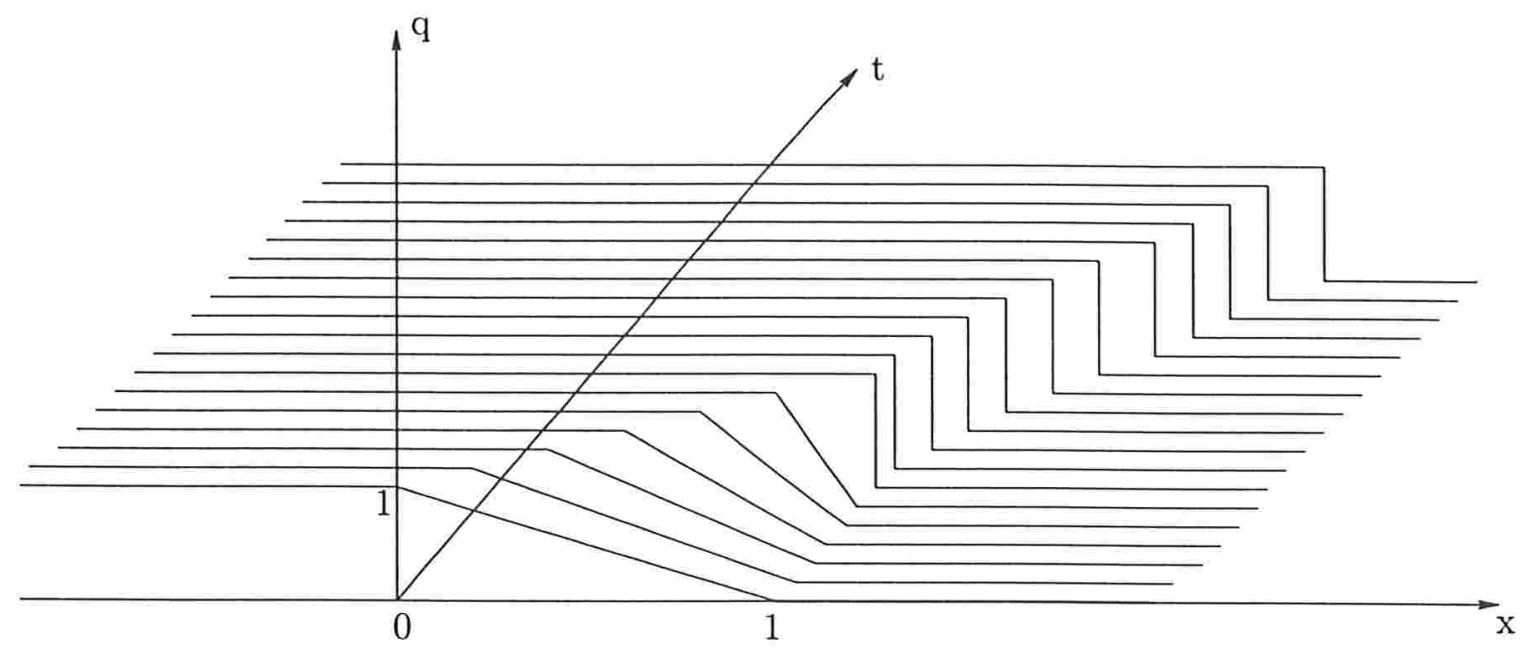

Figura 2.4: Solução fraca da equação de Burgers com dado inicial (2.27).

também tem grande importância prática, pois algumas das técnicas numéricas mais usadas para estudar leis de conservação estão baseadas na resolução de uma seqüência de problemas de Riemann. Além disso, estas técnicas numéricas são a base para provas de existência geral.

Consideremos a equação de Burgers com o dado inicial (2.30). A forma da solução depende da relação entre $q_{e}$ e $q_{d}$.

Caso I: $q_{e}>q_{d}$.

Neste caso existe uma única solução fraca,

$$
q(x, t)=\left\{\begin{array}{lll}
q_{e}, & \text { se } x<s t, \\
q_{d}, & \text { se } x>s t,
\end{array}\right.
$$

onde $s=\left(q_{e}+q_{d}\right) / 2$ é a velocidade do choque. Observe que as curvas características em cada uma das regiões onde $q$ é constante "entram" na onda de choque (veja Figura 2.5), quando o tempo evolui.

Caso II: $q_{e}<q_{d}$. 


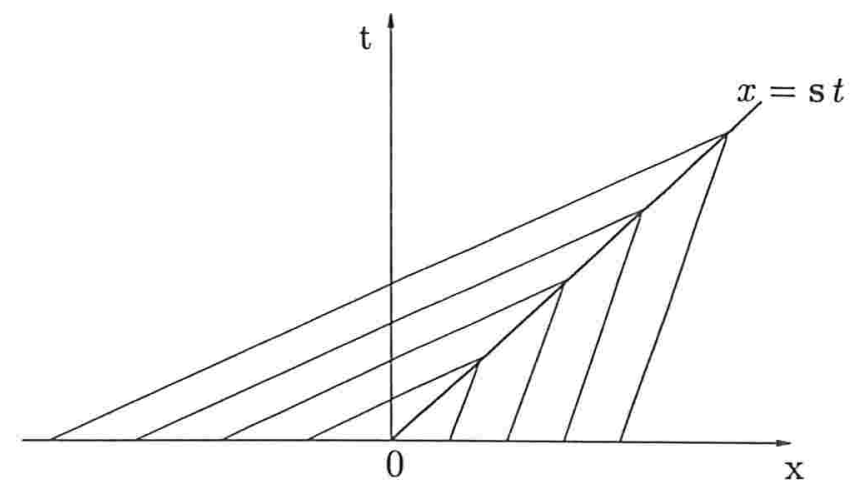

Figura 2.5: Características e choque na solução fraca.

Neste caso as características não preenchem o plano como podemos ver na Figura 2.6. Resta portanto, construir a solução nos pontos por onde não passam características. Isto pode ser feito de duas maneiras pelo menos. Ou pela introdução de uma onda de rarefação com $s=$ $\left(q_{e}+q_{d}\right) / 2$, onde podemos observar que as características "saem" do choque (Figura 2.7-(a)), ou pela introdução de um leque de rarefação, como mostramos na Figura 2.7-(b)

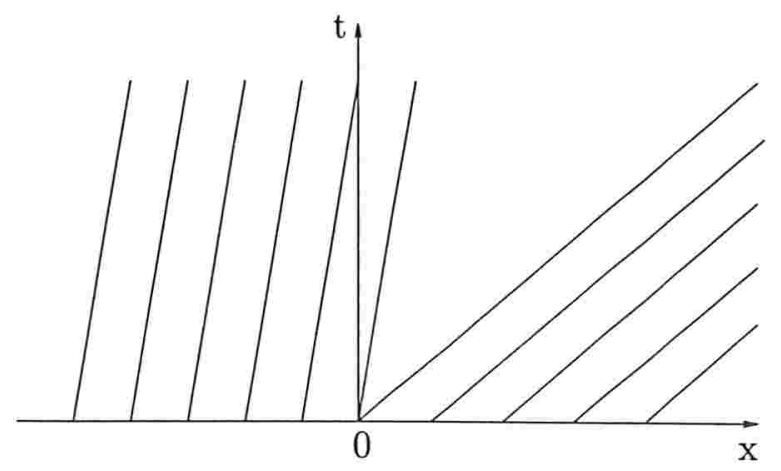

Figura 2.6: Características no plano da equação de Burgers para o caso II.

A solução com onda de rarefação é dada por (2.31) e no caso da onda de rarefação por

$$
q(x, t)=\left\{\begin{array}{lll}
q_{e} & , & \text { se } x<q_{e} t \\
x / t & , & \text { se } q_{e} t \leq x \leq q_{d} t \\
q_{d}, & \text { se } x>q_{d} t .
\end{array}\right.
$$




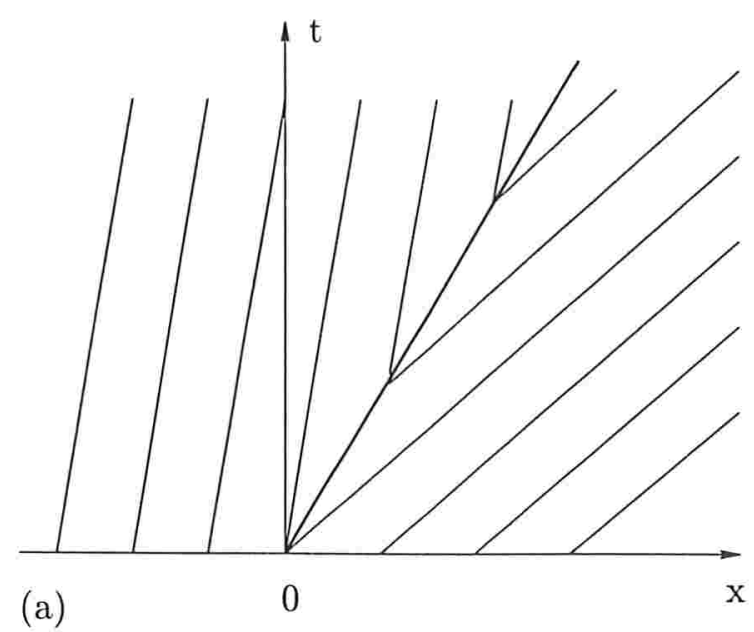

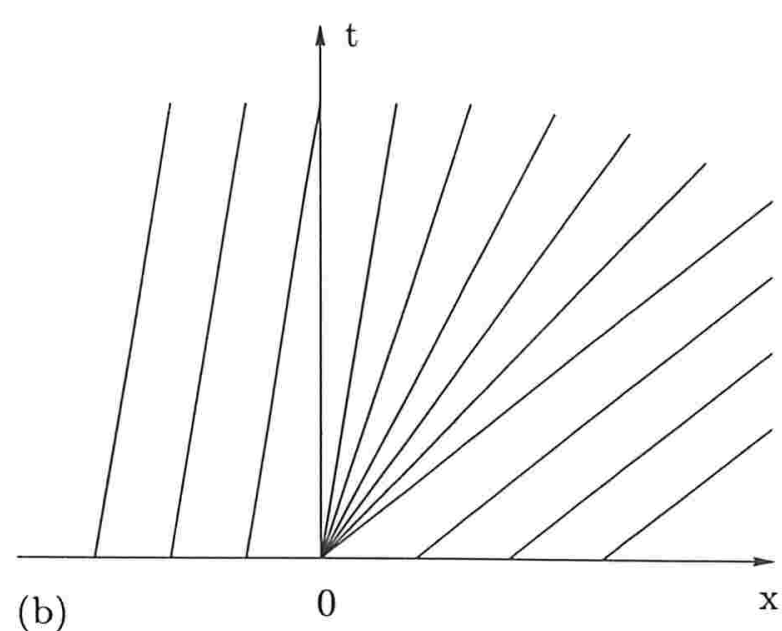

(b)

Figura 2.7: (a) Características no plano exibindo uma onda de rarefação. (b) Características no plano exibindo um leque de rarefação.

Mais ainda, neste caso existem infinitas soluções fracas. Por exemplo

$$
q(x, t)= \begin{cases}q_{e}, & \text { se } x<s_{m} t, \\ q_{m}, & \text { se } s_{m} t \leq x \leq q_{m} t, \\ x / t & , \text { se } q_{m} t \leq x \leq q_{d} t \\ q_{d}, & \text { se } x>q_{d} t\end{cases}
$$

é também uma solução fraca para qualquer $q_{m}$ com $q_{e} \leq q_{m} \leq q_{d}$ e $s_{m}=\left(q_{e}+q_{m}\right) / 2$.

Deduziremos a seguir a solução com onda de rarefação para um problema escalar convexo geral, (2.17) com dado inicial (2.30) e $q_{e}<q_{d}$.

Como nosso dado inicial ocorre em $x=0$, podemos tomar qualquer solução fraca $q(x, t)$ de (2.17) e formar uma familia parametrizada de soluções

$$
q_{\lambda}(x, t):=q(\lambda x, \lambda t) .
$$

Se desejarmos que nosso problema tenha uma única solução, então $q$ deve ter a forma

$$
q(x, t):=\tilde{q}(x / t) .
$$

Substituindo esta equação em (2.17) temos

$$
-\frac{x}{t^{2}} \tilde{q}^{\prime}+\frac{1}{t} f^{\prime}(\tilde{q}) \tilde{q}^{\prime}=0,
$$


de onde,

$$
\frac{1}{t} \tilde{q}^{\prime} \cdot\left(f^{\prime}(\tilde{q})-\frac{x}{t}\right)=0
$$

Portanto, ou $\tilde{q}$ é constante ou

$$
f^{\prime}(\tilde{q}(x / t))=x / t .
$$

Neste caso, usamos o fato que $f^{\prime \prime}>0$ para deduzir que $f^{\prime}$ é inversível e obtermos

$$
\tilde{q}(x / t)=f^{\prime-1}(x / t) .
$$

Portanto a solução com onda de rarefação é dada por

$$
q(x, t)= \begin{cases}q_{e} & , \quad \text { se } x<f^{\prime}\left(q_{e}\right) t \\ f^{\prime-1}(x / t) & , \quad \text { se } f^{\prime}\left(q_{e}\right) t \leq x \leq f^{\prime}\left(q_{d}\right) t \\ q_{d} & , \quad \text { se } x>f^{\prime}\left(q_{d}\right) t\end{cases}
$$

Observemos que com a introdução do conceito de solução fraca é possível obter diversas soluções globais para um mesmo problema. Entretanto, nem todas elas são fisicamente relevantes. Veremos a seguir um critério adicional para discernir qual delas é fisicamente relevante.

\subsection{Condições de Entropia}

Sabemos que a solução com onda de choque do caso I da seção anterior, chamada de onda de compressão, ocorre em dinâmica de gases. As características, ao serem percorridas no sentido de $t$ crescente, entram na onda de choque e "desaparecem", Figura 2.5, acarretando perda de informações (o processo é irreversível), gerando um crescimento da Entropia. Isto é fisicamente viável.

No caso II, a solução, onda de rarefação, nunca ocorre em dinâmica de gases. A sua ocorrência acarretaria em decrescimento da Entropia, o que é fisicamente inviável, havendo neste a geração espontânea de informações. Percebemos ser este um critério natural para selecionar uma das muitas soluções fracas que possa ter a lei de conservação. Assim, denominaremos soluções entrópicas, aquelas soluções fracas que geram um crescimento de entropia.

Agora apresentaremos nossa primeira versão da condição de entropia. 
Condição de Entropia (Versão I, Condição de choque de Lax ${ }^{2}$ ) : Uma descontinuidade que se propaga com velocidade $s$ dada por (2.23) satisfaz a condição de entropia, se

$$
f^{\prime}\left(q_{e}\right)>s>f^{\prime}\left(q_{d}\right)
$$

Para $f$ convexa, a velocidade $s$ da descontinuidade, dada pela equação (2.23), deve estar entre $f^{\prime}\left(q_{e}\right)$ e $f^{\prime}\left(q_{d}\right)$; desta forma a equação $(2.35)$ se reduz simplesmente à condição $f^{\prime}\left(q_{e}\right)>f^{\prime}\left(q_{d}\right)$ o qual, novamente pela convexidade, implica em $q_{e}>q_{d}$.

Podemos mostrar que a equação (2.17) com $f(q)$ convexa e $q_{0}(x)$ limitada e localmente integrável, possui uma única solução entrópica. Esta solução pode ser clássica, diferenciável numa vizinhança do dado inicial e, eventualmente, vir a desenvolver descontinuidades (choques), como por exemplo na Figura 2.3-(b), o choque se forma no instante $T_{0}=1$. Até este instante a solução era clássica. Vemos que o processo até $T_{0}$ é reversível, uma vez que podemos obter qualquer dado inicial a partir da solução em $(x, t)$, bastando para isto voltar aos dados iniciais ao longo da curva característica que passa por $(x, t)$. A partir da formação do choque, o processo se torna irreversível pois as curvas características passam a ser "engolidas" pelo choque. Assim, num tempo $T_{1}>T_{0}$, todas as informações provenientes de algum intervalo $\left[x_{1}, x_{2}\right]$ se perderam. Portanto, não é possível recuperar estes dados iniciais a partir da solução no tempo $T_{1}$. Isto, a grosso modo, caracteriza a irreversivilidade do processo.

A seguir, damos uma forma mais geral da condição de entropia (2.35), devida a Oleinik [24], a qual se aplica também a funções de fluxo escalares $f$, não convexas. Veja também LeVeque [18].

Condição de Entropia (Versão II) : A solução $q(x, t)$ é uma solução entrópica se todas as descontinuidades tiverem a seguinte propriedade:

$$
\frac{f(q)-f\left(q_{e}\right)}{q-q_{e}} \geq s \geq \frac{f(q)-f\left(q_{d}\right)}{q-q_{d}},
$$

para todo $q$ entre $q_{e}$ e $q_{d}$.

Para $f$ convexa, esta condição se reduz a (2.35). Outra forma de condição de entropia é baseada no espalhamento das características num leque de rarefação. Se $q(x, t)$ for uma função

\footnotetext{
${ }^{2}$ Esta condição pode ser generalizada para sistemas, ver por exemplo Lax [14], Renardy e Rogers [26] e Smoller [28].
} 
crescente de $x$ em alguma região, então as características se espalham para fora se $f^{\prime \prime}(x)>0$. A taxa de espalhamento pode ser quantificada e é dada pela condição a seguir, também devida a Oleinik [24] (veja também LeVeque [18] e Smoller [28].

Condição de Entropia (Versão III) : A solução $q(x, t)$ é uma solução entrópica se existir uma constante $E>0$ tal que para todo $a>0, t>0$ e $x \in \mathbb{R}$, temos

$$
\frac{q(x+a, t)-q(x, t)}{a}<\frac{E}{t} .
$$

Agora enunciaremos um teorema de existência e unicidade para as soluções entrópicas.

Teorema 2.6.1 (Oleinik) Seja $q_{0} \in L_{\infty}(\mathbb{R})$, e $f \in C^{2}(\mathbb{R})$ com $f^{\prime \prime}>0$ em $\left\{q:|q| \leq\left\|q_{0}\right\|_{\infty}\right\}$. Então existe uma solução ${ }^{3} q$ de (2.17), (2.18) com as seguintes propriedades:

1. $|q(x, t)| \leq\left\|q_{0}\right\|_{\infty} \equiv M, \quad(x, t) \in \mathbb{R} \times \mathbb{R}^{+} ;$

2. Existe uma constante $E>0$, dependendo somente de $M, \mu=\min \left\{f^{\prime \prime}(q):|q| \leq\left\|q_{0}\right\|_{\infty}\right\}$ e $A=\max \left\{\left|f^{\prime}(q)\right|:|q| \leq\left\|q_{0}\right\|_{\infty}\right\}$, tais que para todo $a>0, t>0$ e $x \in \mathbb{R}$

$$
\frac{q(x+a, t)-q(x, t)}{a}<\frac{E}{t}
$$

3. $q$ é estável e depende continuamente de $q_{0}$ no seguinte sentido: Se $q_{0}, \bar{q}_{0} \in L_{\infty}(\mathbb{R}) \cap L_{1}(\mathbb{R})$ com $\left\|\bar{q}_{0}\right\|_{\infty} \leq\left\|q_{0}\right\|_{\infty}, \bar{q}$ é a solução correspondente construída de (2.17) com dado inicial $\bar{q}_{0}$, então para todo $x_{1}$ e $x_{2} \in \mathbb{R}$, com $x_{1}<x_{2}$, e todo $t>0$,

$$
\int_{x_{1}}^{x_{2}}|q(x, t)-\bar{q}(x, t)| d x \leq \int_{x_{1}+A t}^{x_{2}+A t}\left|q_{0}(x)-\bar{q}(x)\right| d x .
$$

Teorema 2.6.2 Seja $f \in C^{2}, \quad f^{\prime \prime}>0$, e seja $q$ e $\bar{q}$ duas soluções satisfazendo a condição (2.38). Então $q=\bar{q}$ para $t>0$.

A prova destes teoremas pode ser encontrada em Oleinik [24] e Smoller [28].

\footnotetext{
${ }^{3}$ entendemos por solução, uma função $q(x, t)$ que satisfaz (2.22).
} 
Um outro resultado sobre existência e unicidade de soluções, no espaço $B V$ (o espaço de funções que são localmente integráveis e cujas derivadas generalizadas são localmente medíveis), pode ser encontrado em Vol'pert [31].

Para analizarmos este capítulo, comentamos que resultados de existência e unicidade para leis de conservação hiperbólicas com termos forçantes (2.12) são dados por Oleinik [24].

Soluções fracas desta equação são definidas de maneira análoga à equação homogênea, isto é, elas devem satisfazer a equação integral

$$
\int_{0}^{\infty} \int_{-\infty}^{+\infty}\left[\phi_{t} q+\phi_{x} f(q)+\phi \psi(q)\right] d x d t+\int_{-\infty}^{+\infty} \phi(x, 0) q(x, 0) d x=0,
$$

para toda função teste $\phi$. 


\section{Método de Propagação de Ondas}

Neste Capítulo, apresentaremos o Método de Propagação de Ondas para resolver leis de conservação hiperbólicas escalares com termo forçante,

$$
q_{t}+f(q)_{x}=\psi(q)
$$

Este método baseia-se na resolução de problemas de Riemann. Inicialmente serão empregadas discretizações de primeira ordem as quais depois de agregar-lhes termos de correção e empregar funções limitadoras, o método do tipo Godunov é transformado num método de alta resolução.

Métodos do tipo-Godunov (veja apêndice A) de alta resolução, como o Método de Propagação de Ondas, foram desenvolvidos para capturar, com muita precisão, soluções descontínuas da versão homogênea $(\psi \equiv 0)$ da equação $(3.1)$. Quando $\psi \neq 0$, como será nosso caso, empregaremos um método de passo fracionário conhecido como "divisão de Strang", o qual apresentaremos no próximo capítulo.

Na Seção 3.1, desenvolveremos o Método de Propagação de Ondas para sistemas lineares. Isto será feito para entender e ilustrar melhor o método, com a vantagem de motivar a notação utilizada em sistemas mais gerais. Inicialmente, escreveremos o método de Godunov (que é de primeira ordem) em forma de propagação de ondas. Logo depois, para obter alta resolução, adicionar-lhe-emos termos de correção de segunda ordem, os quias serão modificados pelas funções limitadores de onda, tendo por objetivo evitar oscilações perto das descontinuidades. Desta forma obtemos um método de alta resolução. 
Na Seção 3.2, apresentaremos o método para sistemas não lineares. Neste caso, a linearização do problema é feita usando o "resolvedor de Roe" o qual fornece um conjunto de ondas na forma requerida. As dificuldades que poder-se-iam apresentar no caso de termos ondas de rarefação transônica são resolvidas fixando-se a entropia.

Na última seção, Seção 3.3 apresentaremos o método para o caso escalar e apresentaremos como exemplo a solução numérica da equação de Burgers (2.26)-(2.27), as quais foram resolvidas analiticamente no capítulo anterior.

\subsection{Motivação: Sistemas Lineares}

Começamos esta seção resolvendo o sistema linear hiperbólico de leis de conservação para motivar e entender melhor o Método de Propagação de Ondas.

Consideremos o sistema linear com coeficientes constantes

$$
\begin{aligned}
q_{t}+A q_{x} & =0, \\
q(x, 0) & =q_{0}(x), \quad x \in \mathbb{R},
\end{aligned}
$$

Para o qual a matriz $A, m \times m$ tem autovalores $\lambda_{i}, i=1,2, \ldots, m$, reais e distintos dois a dois (sistema hiperbólico), que ordenamos como

$$
\lambda_{1}<\lambda_{2}<\cdots<\lambda_{m} .
$$

Podemos escrever $A$ como

$$
A=R \Lambda R^{-1}
$$

onde $\Lambda=\operatorname{diag}\left(\lambda_{1}, \ldots, \lambda_{m}\right)$ e $R=\left(r_{1}\left|r_{2}\right| \ldots \mid r_{m}\right)$ é a matriz dos autovetores.

Resolvemos a equação (3.2) fazendo a mudança de variáveis $q=R v$. Fazendo esta mudança na equação (3.2) obtemos

$$
v_{t}+\Lambda v_{x}=0
$$


e lembrando que $\Lambda$ é diagonal, temos

$$
\left(v_{p}\right)_{t}+\lambda_{p}\left(v_{p}\right)_{x}=0, p=1,2, \ldots, m .
$$

Estas são equações lineares de advecção, que já vimos no capítulo anterior, cuja solução é dada por

$$
v_{p}(x, t)=v_{p}\left(x-\lambda_{p} t, 0\right), p=1,2, \ldots, m,
$$

onde o dado inicial para $v_{p}$ é dado por $v(x, 0)=R^{-1} q(x, 0)$.

Voltando à variável original $q(x, t)=R v(x, t)$, vemos que esta pode ser escrita como

$$
q(x, t)=\sum_{p=1}^{m} v_{p}(x, t) r_{p}=\sum_{p=1}^{m} v_{p}\left(x-\lambda_{p} t, 0\right) r_{p} .
$$

Agora, resolveremos o problema de Riemann para a equação (3.2), isto é quando a condição inicial (3.3) é dada por

$$
q_{0}(x)= \begin{cases}q_{l} & , \quad x<0 \\ q_{r} & , \quad x>0 .\end{cases}
$$

Se decompusermos o estado inicial

$$
q_{l}=\sum_{p=1}^{m} \alpha_{p} r_{p} \quad \text { e } \quad q_{r}=\sum_{p=1}^{m} \beta_{p} r_{p}
$$

então teremos que

$$
v_{p}(x, 0)= \begin{cases}\alpha_{p} & , \quad x<0 \\ \beta_{p}, & x>0, \quad p=1, \ldots, m,\end{cases}
$$

e portanto,

$$
v_{p}(x, t)= \begin{cases}\alpha_{p}, & x-\lambda_{p} t<0, \\ \beta_{p}, & x-\lambda_{p} t>0, \quad p=1, \ldots, m .\end{cases}
$$

Se introduzirmos a função $P(x, t)=\max \left\{p \mid x-\lambda_{p} t>0\right\}$, então poderemos escrever a solução como

$$
q(x, t)=\sum_{p=1}^{P(x, t)} \beta_{p} r_{p}+\sum_{p=P(x, t)+1}^{m} \alpha_{p} r_{p} .
$$

$\mathrm{O}$ valor de $q(x, t)$ em um ponto dado é ilustrado na Figura 3.1. Neste caso podemos ver que $v_{1}=\beta_{1}, v_{2}=\beta_{2}, v_{3}=\alpha_{3}$ e $v_{4}=\alpha_{4}$. A solução no ponto ilustrado é

$$
q(x, t)=\beta_{1} r_{1}+\beta_{2} r_{2}+\alpha_{3} r_{3}+\alpha_{4} r_{4}
$$


Observemos que o valor da solução é a mesma em qualquer ponto da "cunha" formado pelas características $x^{\prime}(t)=\lambda_{2}$ e $x^{\prime}(t)=\lambda_{3}$. Quando atravessamos a $p$-ésima característica, o valor de $x-\lambda_{p} t$ passa através de zero e o valor de $v_{p}$ pula de $\alpha_{p}$ para $\beta_{p}$. Os outros $v_{i}$ permanecem constantes.

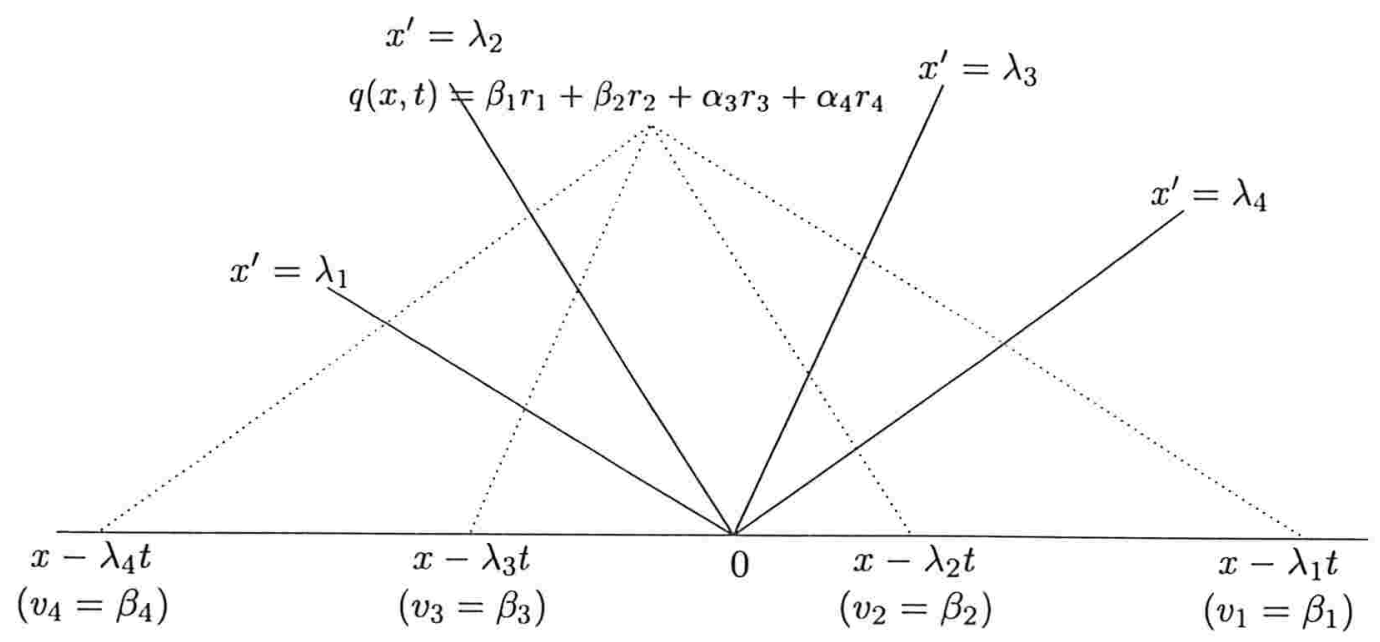

Figura 3.1: Construção da solução para o problema de Riemann em $(x, t)$.

A solução é constante em cada "cunha", como mostramos na Figura 3.1. Através da $p$-ésima característica a solução "salta", sendo o salto dado por

$$
[q]_{p}=\left(\beta_{p}-\alpha_{p}\right) r_{p}
$$

É importante notar que estes saltos satisfazem a condição de Rankine-Hugoniot, pois $f(q)=A q$, isto é,

$$
[f]_{p}=A[q]_{p}=\left(\beta_{p}-\alpha_{p}\right) A r_{p}=\lambda_{p}\left(\beta_{p}-\alpha_{p}\right) r_{p}=\lambda_{p}[q]_{p},
$$

onde vemos que $\lambda_{p}$ é precisamente a velocidade do $p$-ésimo salto.

Agora, alternativamente, podemos re-escrever a solução $q(x, t)$ em termos destes saltos, isto é,

$$
\begin{aligned}
& q(x, t)=q_{l}+\sum_{\lambda_{p}<x / t}\left(\beta_{p}-\alpha_{p}\right) r_{p}, \text { ou } \\
& q(x, t)=q_{r}-\sum_{\lambda_{p}>x / t}\left(\beta_{p}-\alpha_{p}\right) r_{p}
\end{aligned}
$$


A seguir, expressaremos o método de Godunov padrão (veja Apêndice A) para a equação linear (3.2) na forma de propagação de ondas.

Consideremos uma malha unidimensional com valores médios $q_{i}^{n}$ centrados no $n$-ésimo nível de tempo $t_{n}(\Delta t \equiv k)$ e na $i$-ésima célula da malha $C_{i} \equiv\left[x_{i-1 / 2}, x_{i-1 / 2}+\Delta x\right], \Delta x \equiv h$, e $x_{i-1 / 2}=x_{i}-h / 2, x_{i+1 / 2}=x_{i}+h / 2$, como mostramos na Figura 3.2

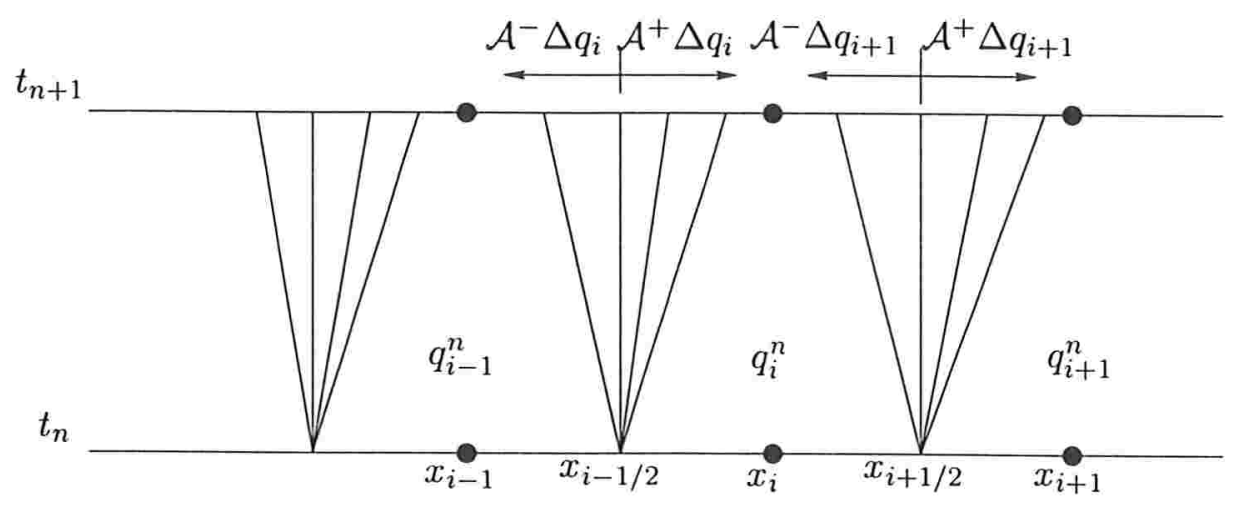

Figura 3.2: Discretização do problema.

Cada $\Delta q_{i}=q_{i}^{n}-q_{i-1}^{n}$ pode ser decomposto como uma combinação linear dos autovetores $r_{p}$ de $A$,

$$
\Delta q_{i}=\sum_{p=1}^{m} \bar{\alpha}_{i}^{p} r_{p}=\sum_{p=1}^{m} \mathcal{W}_{i}^{p},
$$

definindo as ondas $\mathcal{W}_{i}^{p}=\bar{\alpha}_{i}^{p} r_{p}$, cuja velocidade de propagação é justamente $\lambda_{p}$.

Definamos agora,

$$
\lambda^{p+}=\max \left(\lambda^{p}, 0\right), \quad \lambda^{p-}=\min \left(\lambda^{p}, 0\right),
$$

e as matrizes

$$
\Lambda^{ \pm}=\operatorname{diag}\left(\lambda^{p \pm}\right), \quad A^{ \pm}=R \Lambda^{ \pm} R^{-1} .
$$

No Método de Godunov, quando resolvemos o problema de Riemann com dados esquerdo e direito $q_{i}^{n}$ e $q_{i+1}^{n}$, precisamos do valor constante $q^{*}\left(q_{i}^{n}, q_{i+1}^{n}\right)$, na interface $x=x_{i+1 / 2}$. Supondo que $x_{i+1 / 2}=0$, então pelas equações (3.4)-(3.5) este valor pode ser escrito de duas maneiras 
diferentes

$$
q^{*}\left(q_{i}^{n}, q_{i+1}^{n}\right)=q_{i}^{n}+\sum_{\lambda_{p}<0} \mathcal{W}_{i}^{p}=q_{i+1}^{n}-\sum_{\lambda_{p}>0} \mathcal{W}_{i}^{p}
$$

O fluxo correspondente nesta interface será, segundo a notação do Método de Godunov,

$$
\begin{aligned}
F\left(q_{i}^{n}, q_{i+1}^{n}\right) & =A q^{*}\left(q_{i}^{n}, q_{i+1}^{n}\right) \\
& =A q_{i}^{n}+\sum_{\lambda_{p}<0} A \mathcal{W}_{i+1}^{p}=A q_{i+1}^{n}-\sum_{\lambda_{p}>0} A \mathcal{W}_{i+1}^{p} \\
& =A q_{i}^{n}+\sum_{\lambda_{p}<0} A \bar{\alpha}_{i+1}^{p} r_{p}=A q_{i+1}^{n}-\sum_{\lambda_{p}>0} A \bar{\alpha}_{i+1}^{p} r_{p} \\
& =A q_{i}^{n}+\sum_{\lambda_{p}<0} \lambda_{p} \bar{\alpha}_{i+1}^{p} r_{p}=A q_{i+1}^{n}-\sum_{\lambda_{p}>0} \lambda_{p} \bar{\alpha}_{i+1}^{p} r_{p} \\
& =A q_{i}^{n}+\sum_{\lambda_{p}<0} \lambda_{p} \mathcal{W}_{i+1}^{p}=A q_{i+1}^{n}-\sum_{\lambda_{p}>0} \lambda_{p} \mathcal{W}_{i+1}^{p}
\end{aligned}
$$

e portanto,

$$
F\left(q_{i}^{n}, q_{i+1}^{n}\right)=A q_{i}^{n}+A^{-} \Delta q_{i+1}=A q_{i+1}^{n}-A^{+} \Delta q_{i+1} .
$$

Se escolhermos a primeira destas expressões equivalentes para $F\left(q_{i}^{n}, q_{i+1}^{n}\right)$ e tomarmos a segunda para definir $F\left(q_{i-1}^{n}, q_{i}^{n}\right)$, isto é,

$$
F\left(q_{i-1}^{n}, q_{i}^{n}\right)=A q_{i}^{n}-A^{+} \Delta q_{i},
$$

então o método de Godunov para o sistema linear toma a forma

$$
\begin{aligned}
q_{i}^{n+1} & =q_{i}^{n}-\frac{k}{h}\left[F_{i+1}^{n}-F_{i}^{n}\right], \text { onde } F_{i+1}^{n}:=F\left(q_{i}^{n}, q_{i+1}^{n}\right), \\
& =q_{i}^{n}-\frac{k}{h}\left[A^{-} \Delta q_{i+1}+A^{+} \Delta q_{i}\right] .
\end{aligned}
$$

Das expressões (3.9) e (3.10), lembrando que no caso linear $f(q)=A q$, temos

$$
\begin{aligned}
& A^{+} \Delta q_{i}=f\left(q_{i}^{n}\right)-F_{i}^{n}=\sum_{\lambda_{p}>0} \lambda_{p} \mathcal{W}_{i}^{p}, \\
& A^{-} \Delta q_{i}=F_{i}^{n}-f\left(q_{i-1}^{n}\right)=\sum_{\lambda_{p}<0} \lambda_{p} \mathcal{W}_{i}^{p} .
\end{aligned}
$$

A equação (3.12) tem uma interpretação física em termos de propagação de ondas; $\frac{k}{h} A^{+} \Delta q_{i}$ é a soma de termos da forma $\frac{k \lambda_{p}}{h} \mathcal{W}_{i}^{p}$, os quais representam ondas que se movem para dentro 
da $i$-ésima célula provenientes do lado esquerdo (veja Figura 3.2). Cada parcela fornece a contribuição média dada pelas ondas provenientes da interface existente entre as células centradas em $x_{i-1}$ e $x_{i} ; \mathcal{W}_{i}^{p}$ é o valor do salto em $q, k \lambda_{p}$ é a distância propagada para dentro da célula e a divisão por $h$ nos dá a fração da célula coberta. Similarmente, $\frac{k}{h} A^{-} \Delta q_{i+1}$ fornece a contribuição média devido às ondas $\mathcal{W}_{i+1}^{p}$ propagando-se para dentro da célula centrada em $x_{i}$, provenientes da interface localizada à sua direita (veja Figura 3.2).

Existem algumas vantagens da forma (3.12) em termos da implementação numérica sobre a forma dada pela diferença de fluxos (3.11). Depois de calcularmos as ondas e suas velocidades é fácil calcularmos $A^{-} \Delta q$ e $A^{+} \Delta q$ somando seus produtos. Calcular o "fluxo de Godunov" $F_{i}^{n}$, tipicamente requer calcular $f\left(q_{i}^{n}\right)$ e depois modificar por $A^{-} \Delta q$. Outra das vantagens da forma (3.12) sobre a forma (3.11) é que esta permite a solução de problemas hiperbólicos que não estão em forma de conservação. Neste caso, ou quando as equações não são lineares, podemos generalizar o Método de Godunov (3.12) definindo

$$
\begin{aligned}
& \mathcal{A}^{+} \Delta q_{i}=\sum_{\lambda_{i}^{p}>0} \lambda_{i}^{p} \mathcal{W}_{i}^{p}, \\
& \mathcal{A}^{-} \Delta q_{i}=\sum_{\lambda_{i}^{p}<0} \lambda_{i}^{p} \mathcal{W}_{i}^{p},
\end{aligned}
$$

tal que,

$$
\mathcal{A}^{-} \Delta q_{i}+\mathcal{A}^{+} \Delta q_{i}=f\left(q_{i}^{n}\right)-f\left(q_{i-1}^{n}\right) .
$$

Assim sendo, no caso geral, a equação (3.12) se reescreve como

$$
q_{i}^{n+1}=q_{i}^{n}-\frac{k}{h}\left[\mathcal{A}^{-} \Delta q_{i+1}+\mathcal{A}^{+} \Delta q_{i}\right]
$$

Mais adiante, veremos como introduzir no caso geral estas diferenças em termos de propagação de ondas.

\subsubsection{Correções de Segunda Ordem e Limitadores de Onda}

O método (3.12) é de primeira ordem, mas ele pode ser estendido para um método de alta resolução agregando-lhe um termo de correção. Mostraremos como isto pode ser feito primeiramente no contexto de equação linear de adveç̧ão $m$-dimensional, (3.2). A forma do método será

$$
q_{i}^{n+1}=q_{i}^{n}-\frac{k}{h}\left[A^{+} \Delta q_{i}+A^{-} \Delta q_{i+1}\right]-\frac{k}{h}\left[\tilde{F}_{i+1}-\tilde{F}_{i}\right] .
$$


O fluxo corretor $\tilde{F_{i}^{n}}$ é definido em termos das ondas $\mathcal{W}_{i}^{p}$ e suas velocidades $\lambda_{p}$ que se originam do $i$-ésimo problema de Riemann. Os termos de correção de segunda ordem têm a forma

$$
\tilde{F}_{i}^{n}=\frac{1}{2} \sum_{p=1}^{m}\left|\lambda_{i}^{p}\right|\left(1-\frac{k}{h}\left|\lambda_{i}^{p}\right|\right) \mathcal{W}_{i}^{p} .
$$

Substituindo-se as diferenças de fluxos na equação (3.16) e colocando-se tudo em forma matricial temos

$$
\begin{aligned}
q_{i}^{n+1} & =q_{i}^{n}-\frac{k}{h}\left[A^{-} q_{i+1}^{n}+|A| q_{i}^{n}-A^{+} q_{i-1}^{n}\right]-\frac{k}{2 h}\left[A^{+}-A^{-}\right]\left(q_{i+1}^{n}-2 q_{i}^{n}+q_{i-1}^{n}\right) \\
& +\frac{k^{2}}{2 h^{2}}|A|^{2}\left(q_{i+1}^{n}-2 q_{i}^{n}+q_{i-1}^{n}\right),
\end{aligned}
$$

onde usamos as equações (3.13), (3.14), $|A|=A^{+}-A^{-}$e $|A|^{2}=R|\Lambda|^{2} R^{-1}$. Agora, calculando e usando o fato que $A=A^{+}+A^{-}$, temos

$$
q_{i}^{n+1}=q_{i}^{n}-\frac{k}{2 h} A\left(q_{i+1}^{n}-q_{i-1}^{n}\right)+\frac{k^{2}}{2 h^{2}}|A|^{2}\left(q_{i+1}^{n}-2 q_{i}^{n}+q_{i-1}^{n}\right),
$$

que é o método de Lax-Wendroff para sistemas lineares. Esse método, como sabemos, apresenta oscilações espúrias perto das descontinuidades (veja LeVeque [18]), portanto há necessidade de introduzirmos funções limitadoras que modifiquem a equação (3.17) perto das descontinuidades. A decomposição em ondas nos permite aplicar funções limitadoras, comparando a $p$-ésima onda com a $p$-ésima originada do problema de Riemann vizinho.

Dependendo da interpretação das funções limitadoras, estas são chamadas geralmente limitadores de fluxo ou limitadores de inclinação veja LeVeque [18]. No presente contexto, estas serão chamadas limitadores de onda, pois é a magnitude da onda $\mathcal{W}_{i}^{p}$ da equação (3.16) que será modificada. Fazemos isto substituindo cada $\mathcal{W}_{i}^{p}$ por uma versão limitada $\widetilde{\mathcal{W}}_{i}^{p}$, a qual é obtida comparando $\mathcal{W}_{i}^{p}$ com a $p$-onda correspondente $\mathcal{W}_{i-1}^{p}$ ou $\mathcal{W}_{i+1}^{p}$, que originam-se da solução do problema de Riemann vizinho da esquerda ou direita. A direção é escolhida como sendo a direção "contra o fluxo", isto é, olhamos para a esquerda se $\lambda_{i}^{p}>0$ e para a direita se $\lambda_{i}^{p}<0$.

Assumamos $\lambda_{i}^{p}>0$ tal que na $p$-ésima família comparamos $\mathcal{W}_{i}^{p} \operatorname{com} \mathcal{W}_{i-1}^{p}$. A aproximação usada aqui é projetar a onda vizinha $\mathcal{W}_{i-1}^{p}$ sobre o vetor $\mathcal{W}_{i}^{p}$ e comparar o comprimento deste vetor projetado com o comprimento de $\mathcal{W}_{i}^{p}$, modificando o comprimento de $\mathcal{W}_{i}^{p}$ se for necessário, mas preservando sua direção. Isto é feito colocando : 


$$
\begin{aligned}
& \theta_{i}^{p}=\frac{\mathcal{W}_{i-1}^{p} \cdot \mathcal{W}_{i}^{p}}{\mathcal{W}_{i}^{p} \cdot \mathcal{W}_{i}^{p}}, \\
& \widetilde{\mathcal{W}}_{i}^{p}=\phi\left(\theta_{i}^{p}\right) \mathcal{W}_{i}^{p} .
\end{aligned}
$$

Existe uma grande variedade de funções limitadoras. Alguns limitadores clássicos são :

$$
\begin{aligned}
& \phi(\theta)=\max (0, \min (1, \theta)), \\
& \phi(\theta)=\max (0, \min (1,2 \theta), \min (2, \theta)), \\
& \phi(\theta)=\max \left(0, \min \left(\frac{1+\theta}{2}, 2,2 \theta\right)\right) .
\end{aligned}
$$

Depois de definirmos as ondas limitadas $\widetilde{\mathcal{W}}_{i}^{p}$, os $\tilde{F}_{i}$ usados em (3.16) são dados por (3.17) com $\mathcal{W}_{i}^{p}$ substituído por $\widetilde{\mathcal{W}}_{i}^{p}$, isto é,

$$
\tilde{F}_{i}=\frac{1}{2} \sum_{p=1}^{M_{w}}\left|\lambda_{i}^{p}\right|\left(1-\frac{k}{h}\left|\lambda_{i}^{p}\right|\right) \widetilde{\mathcal{W}}_{i}^{p} .
$$

\subsection{Sistemas Não-Lineares}

Para um sistema não linear de equações $q_{t}+f(q)_{x}=0$, o Método de Propagação de Ondas é essencialmente idêntico ao caso linear da seção anterior. A diferença está na solução dos problemas de Riemann para cada conjunto de dados $\left(q_{i-1}^{n}, q_{i}^{n}\right)$, para os quais devemos produzir

- Um conjunto de ondas $\mathcal{W}_{i}^{p}, p=1,2, \ldots, M_{w}$, que são os saltos em $q$, e suas velocidades associadas $\lambda_{i}^{p}$,

- Diferenças de fluxos, denotados por $\mathcal{A}^{-} \Delta q_{i}$ e $\mathcal{A}^{+} \Delta q_{i}$.

Aqui $M_{w}$, o número de ondas, é muitas vezes igual a $m$, o número de equações do sistema, embora nem sempre tal fato ocorra. Estas ondas são as descontinuidades de salto. A solução verdadeira do problema de Riemann poderia ser usada para definir estas ondas sempre que a solução não contenha ondas de rarefação, pois nesse caso não teríamos descontinuidades. Porém, é mais eficiente usar alguma solução de Riemann aproximada. Um bom "Resolvedor de Riemann" aproximado é o "Resolvedor de Roe", que fornece um conjunto de ondas da forma requerida. 
O resolvedor de Roe consiste em resolver exatamente um problema de Riemann linearizado, isto é, um sistema linear de leis de conservação com $\hat{f}(\hat{q})=A_{i} \hat{q}$, em vez do sistema não linear original, ou seja

$$
\hat{q}_{t}+A_{i} \hat{q}_{x}=0,
$$

onde $A_{i}=A_{i}\left(q_{i-1}^{n}, q_{i}^{n}\right)$ depende dos dados iniciais. Se $A_{i}$ tem autovalores $\lambda_{i}^{p}$ e autovetores $r_{i}^{p}$, então decompomos

$$
q_{i}^{n}-q_{i-1}^{n}=\sum_{p} \alpha_{i}^{p} r_{i}^{p}=\sum_{p} \mathcal{W}_{i}^{p}
$$

As diferenças de fluxos são definidas de maneira análoga às equações (3.13) e (3.14),

$$
\begin{aligned}
& \mathcal{A}^{+} \Delta q_{i}=A_{i}^{+} \Delta q_{i}=\sum_{\lambda_{i}^{p}>0} \lambda_{i}^{p} \mathcal{W}_{i}^{p}, \\
& \mathcal{A}^{-} \Delta q_{i}=A_{i}^{-} \Delta q_{i}=\sum_{\lambda_{i}^{p}<0} \lambda_{i}^{p} \mathcal{W}_{i}^{p} .
\end{aligned}
$$

O problema que se apresenta, neste caso, é como determinamos a matriz $A_{i}$. Roe sugeriu que devemos impor as seguintes condições sobre $A_{i}$ :

$$
\begin{aligned}
& \text { (i) } A_{i}\left(q_{i-1}^{n}, q_{i}^{n}\right)\left(q_{i}^{n}-q_{i-1}^{n}\right)=f\left(q_{i}^{n}\right)-f\left(q_{i-1}^{n}\right), \\
& \text { (ii) } A_{i}\left(q_{i-1}^{n}, q_{i}^{n}\right) \text { é diagonalizável com autovalores reais, } \\
& \text { (iii) } A_{i}(\bar{q}, \bar{q})=f^{\prime}(\bar{q}) \text {, para um } \bar{q} \text { fixo. }
\end{aligned}
$$

Estas condições garantem que nosso método numérico resultante seja conservativo e consistente com o problema hiperbólico original.

A condição (i) implica em

$$
\mathcal{A}^{-} \Delta q_{i}+\mathcal{A}^{+} \Delta q_{i}=f\left(q_{i}^{n}\right)-f\left(q_{i-1}^{n}\right) \text {. }
$$

A condição (ii) é claramente requerida para que o problema $\hat{q}_{t}+A_{i} \hat{q}_{x}=0$ seja hiperbólico.

A condição (iii) garante que o método comporta-se razoavelmente para o caso de soluções suaves. Os termos de correção e limitadores de onda são os mesmos que para o caso linear. 
Se a solução verdadeira do problema de Riemann inclui uma onda de rarefação transônica, por exemplo, no caso escalar $f^{\prime}\left(q_{i-1}^{n}\right)<0<f^{\prime}\left(q_{i}^{n}\right)$, então é necessário modificar as diferenças de fluxo para incluir uma "entropia fixa". Como veremos depois, as ondas $\mathcal{W}_{i}^{p}$ não serão modificadas, somente as diferenças de fluxo, pois as ondas são somente usadas para as correções de segunda ordem.

\section{Exemplo 3.2.1 (Gases isotérmicos)}

Consideremos as equações de gases isotérmicos. Este sistema é similar às equações de Euler, mas somente envolve duas equações para a conservação de massa e de momento, resultando num sistema mais simples para ilustrar as idéias principais.

As equações isotérmicas são

$$
\begin{aligned}
\rho_{t}+(\rho u)_{x} & =0 \\
(\rho u)_{t}+\left(\rho u^{2}+c^{2} \rho\right)_{x} & =0
\end{aligned}
$$

onde $c$ é constante. Estas são as equações padrão para a conservação de massa e momento, mas com a equação de estado simples

$$
p=c^{2} \rho,
$$

que se verifica para um gás ideal se a temperatura for constante.

Calcularemos a matriz de Roe para estas equações isotérmicas. Se fizermos

$$
q=\left(\begin{array}{c}
\rho \\
m
\end{array}\right), \quad f(q)=\left(\begin{array}{c}
m \\
m^{2} / \rho+c^{2} \rho
\end{array}\right), \text { onde } m=\rho u,
$$

então

$$
f^{\prime}(q)=\left(\begin{array}{cc}
0 & 1 \\
c^{2}-m^{2} / \rho^{2} & 2 m / \rho
\end{array}\right)=\left(\begin{array}{cc}
0 & 1 \\
c^{2}-u^{2} & 2 u
\end{array}\right) .
$$

Para calcular o Jacobiano médio satisfazendo (3.19), introduzimos as novas variáveis

$$
z=\rho^{-1 / 2} q \text {, isto é }\left(\begin{array}{c}
z_{1} \\
z_{2}
\end{array}\right)=\left(\begin{array}{c}
\rho^{1 / 2} \\
m / \rho^{1 / 2}
\end{array}\right)=\left(\begin{array}{c}
\rho^{1 / 2} \\
\rho^{1 / 2} u
\end{array}\right) \text {. }
$$


então

$$
q=z_{1} z=\left(\begin{array}{c}
z_{1}^{2} \\
z_{1} z_{2}
\end{array}\right), f(q)=\left(\begin{array}{c}
z_{1} z_{2} \\
c^{2} z_{1}^{2}+z_{2}^{2}
\end{array}\right) .
$$

A mudança de variáveis foi feita para que ambas $\left(q_{l}-q_{r}\right)$ e $\left(f\left(q_{l}\right)-f\left(q_{r}\right)\right)$ possam ser expressas linearmente em $\left(z_{l}-z_{r}\right)$, onde $q_{l}$ e $q_{r}$ são os estados esquerdo e direito do dado inicial para o problema de Riemann. A média é feita em termos da variável $z$ :

$$
\bar{z}=\frac{1}{2}\left(z_{l}+z_{r}\right)=\left(\begin{array}{c}
\bar{z}_{1} \\
\bar{z}_{2}
\end{array}\right)=\frac{1}{2}\left(\begin{array}{c}
\rho_{l}^{1 / 2}+\rho_{r}^{1 / 2} \\
m_{l} / \rho_{l}^{1 / 2}+m_{r} / \rho_{r}^{1 / 2}
\end{array}\right) .
$$

Então é fácil ver que :

$$
\begin{gathered}
q_{l}-q_{r}=\left(\begin{array}{cc}
2 \bar{z}_{1} & 0 \\
\bar{z}_{2} & \bar{z}_{1}
\end{array}\right)\left(z_{l}-z_{r}\right) \equiv B\left(z_{l}-z_{r}\right), \\
f\left(q_{l}\right)-f\left(q_{r}\right)=\left(\begin{array}{cc}
\bar{z}_{2} & \bar{z}_{1} \\
2 c^{2} \bar{z}_{1} & 2 \bar{z}_{2}
\end{array}\right)\left(z_{l}-z_{r}\right) \equiv C\left(z_{l}-z_{r}\right) .
\end{gathered}
$$

Usando [.] para denotar o salto em uma quantidade, temos $[q]=B[z]$ e $[f]=C[z]$, e daí

$$
[f]=C B^{-1}[q] .
$$

Portanto a condição (3.19-i) é satisfeita se tomarmos

$$
A\left(q_{l}, q_{r}\right)=C B^{-1}=\left(\begin{array}{cc}
0 & 1 \\
c^{2}-\bar{z}_{2}^{2} / \bar{z}_{1}^{2} & 2 \bar{z}_{2} / \bar{z}_{1}
\end{array}\right)=\left(\begin{array}{cc}
0 & 1 \\
c^{2}-\bar{u}^{2} & 2 \bar{u}
\end{array}\right),
$$

onde definimos a velocidade média $\bar{u}$ por

$$
\bar{u}=\frac{\bar{z}_{2}}{\bar{z}_{1}}=\frac{\rho_{l}^{1 / 2} u_{l}+\rho_{r}^{1 / 2} u_{r}}{\rho_{l}^{1 / 2}+\rho_{r}^{1 / 2}}
$$

Podemos enxergar a matriz $A$ como sendo a matriz jacobiana $f^{\prime}(q)$ avaliada na velocidade média $\bar{u}$. Claramente, quando $q_{l}=q_{r}=\bar{q}$ e $\bar{z}=z_{l}=z_{r}$, a matriz $A(\bar{q}, \bar{q})$ reduz-se a $f^{\prime}(\bar{q})$. Como os coeficientes de $A$ são funções suaves de $q_{l}$ e $q_{r}$, (3.19-iii) é satisfeita. A condição (3.19-ii) é também satisfeita pois os autovalores e autovetores são dados por

$$
\begin{array}{cc}
\lambda_{1}=\bar{u}-c \quad, \quad \lambda_{2}=\bar{u}-c \\
r_{1}=\left(\begin{array}{c}
1 \\
\bar{u}-c
\end{array}\right), \quad r_{2}=\left(\begin{array}{c}
1 \\
\bar{u}+c
\end{array}\right) .
\end{array}
$$


Como para nosso método precisamos repetir este procedimento para o $i$-ésimo problema de Riemann com dados $q_{i-1}^{n}$ e $q_{i}^{n}$, portanto teremos que

$$
\bar{u}_{i}=\frac{\rho_{i-1}^{1 / 2} u_{i-1}+\rho_{i}^{1 / 2} u_{i}}{\rho_{i-1}^{1 / 2}+\rho_{i}^{1 / 2}} \text {, e } A_{i}=\left(\begin{array}{cc}
0 & 1 \\
c^{2}-\bar{u}_{i}^{2} & 2 \bar{u}_{i}
\end{array}\right),
$$

que como sabemos pode escrever-se como $A_{i}=R_{i} \Lambda_{i} R_{i}^{-1}$ com

$$
R_{i}=\left(\begin{array}{cc}
1 & 1 \\
\bar{u}_{i}-c & \bar{u}_{i}+c
\end{array}\right), \Lambda_{i}=\left(\begin{array}{cc}
\bar{u}_{i}-c & 0 \\
0 & \bar{u}_{i}+c
\end{array}\right), R_{i}^{-1}=\frac{1}{2 c}\left(\begin{array}{cc}
\bar{u}_{i}+c & -1 \\
-\bar{u}_{i}+c & 1
\end{array}\right) \text {. }
$$

Logo, os coeficientes de onda $\alpha_{i}=R_{i}^{-1} \Delta q_{i}$ são

$$
\begin{aligned}
\alpha_{i}^{1} & =\frac{1}{2 c}\left(\left(\bar{u}_{i}+c\right) \Delta q_{i}^{1}-\Delta q_{i}^{2}\right), \\
\alpha_{i}^{2} & =\frac{1}{2 c}\left(\left(-\bar{u}_{i}+c\right) \Delta q_{i}^{1}+\Delta q_{i}^{2}\right) .
\end{aligned}
$$

Vemos que o resolvedor de Roe decompõe $\Delta q_{i}$ em

$$
\begin{aligned}
\mathcal{W}_{i}^{p} & =\alpha_{i}^{p} r_{i}^{p}, \quad p=1,2 \\
\lambda_{i}^{1} & =\bar{u}_{i}-c, \quad \lambda_{i}^{2}=\bar{u}_{i}+c \\
\mathcal{A}^{-} \Delta q_{i} & =A_{i}^{-} \Delta q_{i}=\left(\lambda_{i}^{1}\right)^{-} \alpha_{i}^{1} r_{i}^{1}+\left(\lambda_{i}^{2}\right)^{-} \alpha_{i}^{2} r_{i}^{2} \\
\mathcal{A}^{+} \Delta q_{i} & =A_{i}^{+} \Delta q_{i}=\left(\lambda_{i}^{1}\right)^{+} \alpha_{i}^{1} r_{i}^{1}+\left(\lambda_{i}^{2}\right)^{+} \alpha_{i}^{2} r_{i}^{2} .
\end{aligned}
$$

Pode ser desejável incorporar uma entropia fixa dentro destas expressões. Para tratar uma rarefação transônica, calculamos o estado intermediário

$$
\hat{q}^{n}=q_{i-1}^{n}+\alpha_{i}^{1} r_{i}^{1}=q_{i}^{n}-\alpha_{i}^{2} r_{i}^{2}
$$

Suponhamos por exemplo, que $u_{i-1}-c<0<\hat{u}_{i}-c$. Então poderia existir uma rarefação transônica na primeira onda. Neste caso colocaríamos

$$
\begin{aligned}
& \mathcal{A}^{-} \Delta q_{i}=\left(\frac{\left(\hat{u}_{i}-c\right)-\left(\bar{u}_{i}-c\right)}{\left(\hat{u}_{i}-c\right)-\left(u_{i-1}-c\right)}\right)\left(u_{i-1}-c\right) \mathcal{W}_{i}^{1}, \\
& \mathcal{A}^{+} \Delta q_{i}=\left(f\left(q_{i}^{n}\right)-f\left(q_{i-1}^{n}\right)\right)-\mathcal{A}^{-} \Delta q_{i} .
\end{aligned}
$$

Observemos que as ondas permanecem inalteradas. Para uma discussão de tal caso veja LeVeque [18]. 


\subsection{O Caso Escalar}

Para uma lei de conservação escalar a condição (3.19-i) determina $a=A\left(q_{i-1}^{n}, q_{i}^{n}\right)$ de maneira única como

$$
a=\frac{f\left(q_{i}^{n}\right)-f\left(q_{i-1}^{n}\right)}{q_{i}^{n}-q_{i-1}^{n}} .
$$

O problema linearizado é a equação de advecção escalar $\hat{q}_{t}+a \hat{q}_{x}=0$ e a solução de Riemann aproximada consiste do salto $q_{i}^{n}-q_{i-1}^{n}$ que se propaga com velocidade $a$. Observemos que o termo " $a$ " na equação anterior é a velocidade do choque para o problema não linear, e portanto a solução de Riemann "aproximada" é na verdade uma solução fraca exata (embora ela possa violar a condição de entropia).

Sabemos que a desvantagem da linearização de Roe é que ela somente considera descontinuidades como solução de Riemann aproximada, não considera ondas de rarefação, e isto pode conduzir à violação da condição da condição de entropia. Soluções de Riemann que violam a condição de entropia só acontecem no caso de ondas de rarefação transônica, no qual $f^{\prime}\left(q_{i-1}^{n}\right)<0<f^{\prime}\left(q_{i}^{n}\right)$. Para evitar tal situação é necessário introduzir uma entropia fixa, como fizemos no exemplo do capítulo anterior.

\section{Exemplo 3.3.1 . Equação de Burgers.}

A equação de Burgers unidimensional é

$$
q_{t}+\left(\frac{1}{2} q^{2}\right)_{x}=0
$$

Nossa equação linearizada é $q_{t}+A_{i} q_{x}=0$ com

$$
A_{i}=\frac{\frac{1}{2}\left(f\left(q_{i}^{n}\right)\right)^{2}-\frac{1}{2}\left(f\left(q_{i-1}^{n}\right)\right)^{2}}{q_{i}^{n}-q_{i-1}^{n}}=\frac{1}{2}\left(q_{i-1}^{n}+q_{i}^{n}\right) .
$$

Então para esta equação escalar temos que $\lambda_{i}^{1}=A_{i}$ é a velocidade de choque correta no caso em que a solução é uma onda de choque, e assim temos que

$$
\begin{aligned}
\mathcal{W}_{i}^{p} & =q_{i}^{n}-q_{i-1}^{n}, \\
\lambda_{i}^{1} & =\frac{1}{2}\left(q_{i-1}^{n}+q_{i}^{n}\right), \\
\mathcal{A}^{-} \Delta q_{i} & =\min \left(\lambda_{i}^{1}, 0\right) \mathcal{W}_{i}^{p}, \\
\mathcal{A}^{+} \Delta q_{i} & =\max \left(\lambda_{i}^{1}, 0\right) \mathcal{W}_{i}^{p} .
\end{aligned}
$$


Observemos que $\mathcal{A}^{-} \Delta q_{i}$ ou $\mathcal{A}^{+} \Delta q_{i}$ deve ser zero. Isto funcionará bem, exceto no caso de uma rarefação transônica, na qual a informação propaga-se em ambas direções. Neste caso, incluiremos a entropia fixa. Se $q_{i-1}^{n}<0$ e $q_{i}^{n}>0$, então em lugar das diferenças de fluxo da equação (3.21) usamos

$$
\begin{aligned}
& \mathcal{A}^{-} \Delta q_{i}=-\frac{1}{2}\left(q_{i-1}^{n}\right)^{2} \\
& \mathcal{A}^{+} \Delta q_{i}=\frac{1}{2}\left(q_{i}^{n}\right)^{2}
\end{aligned}
$$

com a mesma onda e velocidade. Em quaisquer dos casos temos

$$
\mathcal{A}^{-} \Delta q_{i}+\mathcal{A}^{+} \Delta q_{i}=\frac{1}{2}\left(\left(q_{i}^{n}\right)^{2}-\left(q_{i-1}^{n}\right)^{2}\right)=f\left(q_{i}^{n}\right)-f\left(q_{i-1}^{n}\right) .
$$

A seguir apresentaremos a solução numérica da equação de Burgers com dado inicial (2.27), Figura 3.3. Também podemos ver aqui a solução exata para este problema dada pelas equações (2.28)-(2.29). A solução numérica foi calculada usando passos de tempo variáveis e um espaçamento de malha $\Delta x=h=0.005$. Observemos que o método captura bem o choque. 


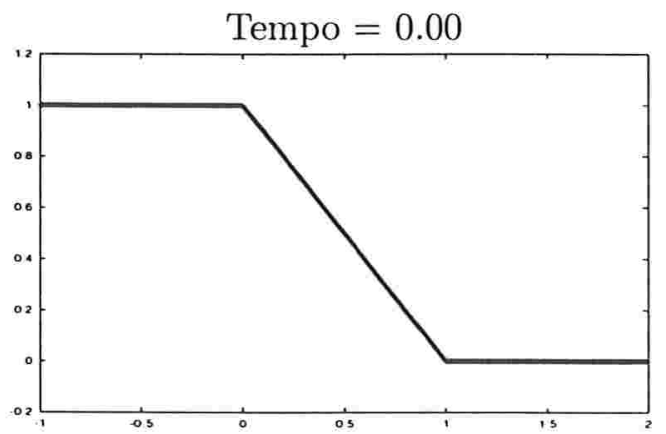

(a)

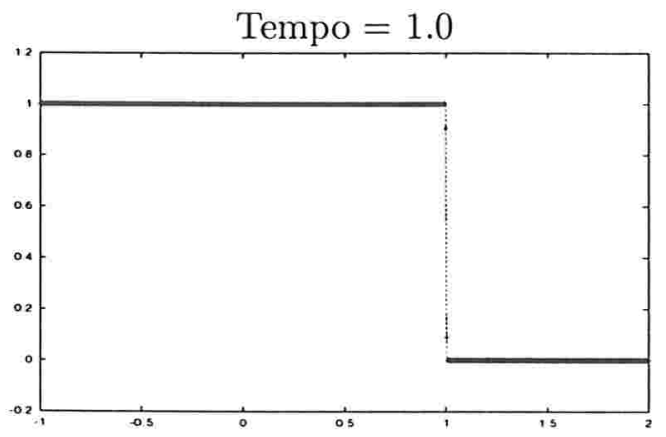

(c)

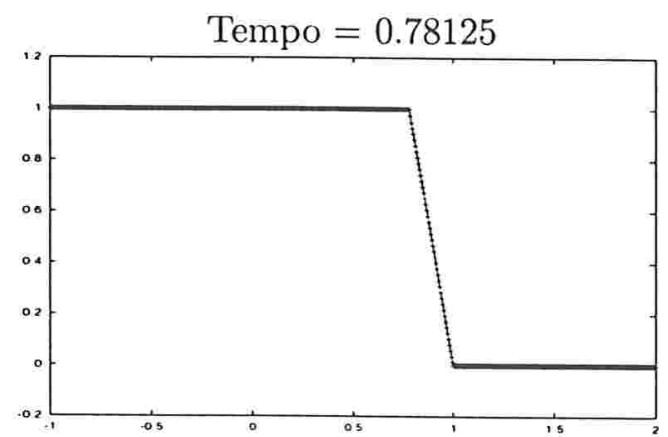

(b)

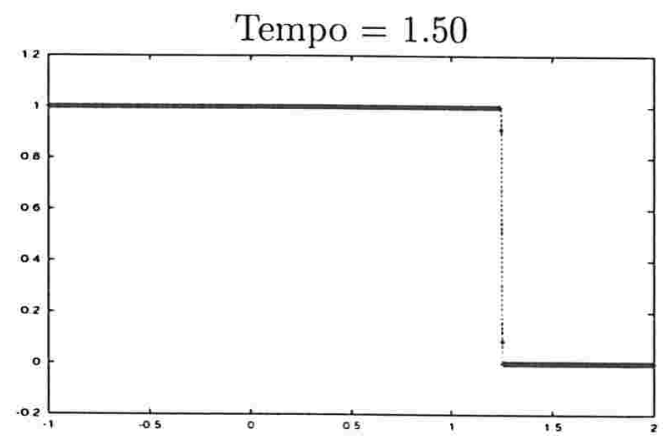

(d)

Figura 3.3: (a)-Dado inicial. (b),(c) e (d)-Solução numérica (com pontos) e exata (com linhas) no tempo $t=1.50$, com $\Delta t=k=0.8 h$. 


\section{O Software CLAWPACK}

CLAWPACK (Conservation LAWs PACKage) é um pacote de subrotinas em FORTRAN para resolver sistemas hiperbólicos de leis de conservação dependentes do tempo em uma, duas ou em três dimensões. O pacote foi desenvolvido por Randall LeVeque do Departamento de Matemática Aplicada da Universidade de Washington.

Neste capítulo, falaremos sobre o CLAWPACK, mas centrar-nos-emos no caso unidimensional, que é o caso de nosso interesse. Apenas faremos referência aos casos bidimensional e tridimensional. Descreveremos também os algoritmos para a resolução da equação (3.1), com $f(q)=\frac{1}{n} q^{n}$ e $\psi(q)=q^{m}$.

Na Seção 4.1, trataremos de como obter o pacote, de como ele está estruturado e organizado nos diferentes diretórios e subdiretórios e, na Seção 4.2, falaremos sobre o tipo de equações que se pode resolver com o CLAWPACK.

Na Seção 4.3, falaremos sobre as condições de fronteira e sobre as variáveis auxiliares e, por último, na Seção 4.4, sobre os termos forçantes e sobre de como o CLAWPACK lida com este tipo de equação e com as possíveis complicações tais como termos forçantes rígidos. 


\subsection{Estrutura e organização do CLAWPACK}

O pacote CLAWPACK pode ser obtido gratuitamente por intermédio da netlib. Para obtermos o índice dos arquivos disponíveis devemos enviar um e-mail para: netlib@research.att.com, com a seguinte mensagem

$$
\text { send pdes/claw/index }
$$

e para obtermos todos os arquivos, colocamos a mensagem

$$
\text { send all from pdes/claw. }
$$

Se só desejarmos uma parte do pacote trocamos "all" pelo nome de um dos subdiretórios. Em particular, o pacote básico está em pdes/claw/clawpack, e enquanto que pdes/claw/applications contém uma grande variedade de aplicações que podem ser acessadas individualmente. Alternativamente, podemos obter os arquivos via ftp na rede mundial de computadores no URL

$$
\text { ftp://netlib.bell-labs.com/netlib/pdes/claw/index.html. }
$$

Também é possível obtê-lo no endereço

$$
\text { http://www . amath. washington.edu/ rjl/clawpack.html . }
$$

Se estivermos interessados em receber e-mails acerca de melhorias, erros, etc. do pacote, devemos enviar o seguinte e-mail para a lista de usuários

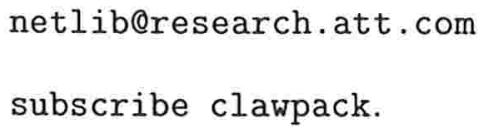

O CLAWPACK está organizado em vários diretórios. O diretório principal é claw (o qual em netlib é pdes/claw), e ele contém os seguintes subdiretórios:

- claw/clawpack - Contém o software CLAWPACK básico e exemplos mínimos de seu uso.

- claw/applications - Contém muitos exemplos de programas e dados para diferentes aplicações. Este, por sua vez, está organizado em subdiretórios para cada aplicação, de tal forma que é possível selecionar um conjunto de aplicações sem a necessidade de se entrar no diretório applications. 
- claw/doc - Contém a documentação na forma de arquivos postscript. Estes tomam a forma de uma apostila que trata sobre vários aspectos do software.

O diretório básico claw/clawpack contém o software propriamente dito e tem subdiretórios 1d, 2d e 3d, contendo os algoritmos em uma, duas e três dimensões respectivamente. Existe também um subdiretório limiters que contém funçōes limitadoras usadas em todas as dimensões.

Cada um dos subdiretórios 1d, 2d e 3d está organizado de forma semelhante. Por exemplo, 1d contém outros subdiretórios:

- claw1 - Contém a subrotina básica claw1.f (chamada pelo usuário) e outras subrotinas chamadas pelo claw1.

- bc - Contém rotinas de condições de fronteira gerais usadas em muitas aplicações, por exemplo extrapolação e condições de fronteira periódicas.

- misc - Contém subrotinas que podem ser usadas além das aplicações particulares, por exemplo, na saída das soluções na forma requerida pelo matlab ou gnuplot.

- matlab - Contém alguns m-arquivos matlab básicos que podem ser usados para visualizar resultados de muitos exemplos.

- example - Um exemplo simples de rotina ilustrando o uso do CLAWPACK.

Neste trabalho não fizemos uso do subdiretório matlab, em seu lugar usamos o gnuplot para visualizar nossos gráficos.

O diretório claw/applications está organizado segundo aplicações particulares. Alguns dos subdiretórios são:

- burgers - Equação de Burgers em uma e duas dimensões.

- euler - Equações de Euler da dinâmica de gases.

- advection - Equações de advecção. 
Estes estão tipicamente subdivididos em $1 \mathrm{~d}$ e $2 \mathrm{~d}$ e dentro de cada um deles existe um subdiretório rp que contém o resolvedor de Riemann e outros subdiretórios para resolver problemas particulares ou classes de problemas. Veja claw/applications/index para uma lista de aplicações disponíveis.

Para rodarmos o código, a melhor maneira é trabalharmos primeiramente com os programasexemplos simples incluídos em claw/clawpack. Em uma dimensão, estes encontram-se no diretório claw/clawpack/

1d/example, por exemplo. Este diretório está organizado da mesma maneira como os outros diretórios de aplicação. Nele encontramos seguintes arquivos:

- README - Este arquivo contém as instruções para rodar o código.

- compile - Este arquivo contém as instruções Unix necessárias para compilar o código, utilizando as subrotinas necessárias dos diferentes diretórios. Colocando o comando

$$
\text { compile }
$$

na janela de comandos do unix, o comando $f 77$ será executado para compilar o programa e criar o executável a.out. Para que isto funcione corretamente, a variável ambiente CLAWPACK deve estar corretamente colocada para indicar o caminho correto para as rotinas clawpack. Por exemplo, em nosso sistema (o sistema do IME-USP) colocamos o comando

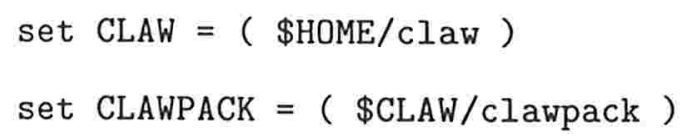

no arquivo .cshrc de tal forma que seja executado cada vez que nós acessemos a rede.

- data - Arquivo de dados que é lido pelo programa, devemos colocar vários parâmetros. Para rodar o código colocar

$$
\text { a. out }<\text { data }
$$

- driver.f - É a rotina principal que chama o CLAWPACK.

- ic.f - Coloca as condições iniciais. 


\subsection{Tipo de equações resolvidas pelo CLAWPACK}

Antes de passarmos a descrever os diferentes algoritmos, vejamos o tipo de equações que podem ser resolvidas com o CLAWPACK.

Em uma dimensão, podemos empregar a rotina claw1 para resolvermos sistemas de equações da forma

$$
\kappa(x) q_{t}+f(q)_{x}=\psi(q . \kappa),
$$

onde $q=q(x, t) \in \mathbb{R}^{m}$. O caso mais simples de uma lei de conservação homogênea acontece quando $\kappa \equiv 1$ e $\psi \equiv 0$,

$$
q_{t}+f(q)_{x}=0 .
$$

Falaremos do termo forçante mais adiante. Para uma discussão da "função capacidade" $\kappa$, ver LeVeque [21], [15].

Sistemas hiperbólicos que não estão em forma conservativa também podem ser resolvidos, por exemplo

$$
q_{t}+A(q, x, t) q_{x}=0 .
$$

Um exemplo desse tipo de equações é dado por LeVeque [21], [15].

Como já vimos no Capítulo 3, o requisito básico para a equação (4.2) é que seja hiperbólica, de forma que um resolvedor de Riemann possa ser usado de tal maneira que, para qualquer dois estados $q_{e}$ e $q_{d}$, retorne um conjunto $M_{w}$ de ondas $\mathcal{W}^{p}$ e velocidades $\lambda^{p}$ satisfazendo

$$
\sum_{p=1}^{M_{w}} \mathcal{W}^{p}=q_{d}-q_{e} \equiv \Delta q .
$$

O resolvedor de Riemann deve também retornar uma diferença de fluxo indo para esquerda $\mathcal{A}^{-} \Delta q$ e uma diferença de fluxo indo para direita $\mathcal{A}^{+} \Delta q$. No caso conservativo (4.2) a condição

$$
\mathcal{A}^{-} \Delta q+\mathcal{A}^{+} \Delta q=f\left(q_{d}\right)-f\left(q_{e}\right)
$$

deve ser satisfeita, e estas "diferenças" definem uma "divisão em diferença de fluxos", (veja as equaçoes (3.9) e (3.10)). No caso não conservativo, e.g. (4.3), a condição (4.4) não precisa ser satisfeita. 
O resolvedor de Riemann deve ser fornecido pelo usuário na forma de uma subrotina rp1. O usuário deve fornecer também uma rotina de condição de fronteira bc1, a qual discutiremos depois, e uma rotina src1 que resolva a equação com termo forçante $q_{t}=\psi(q)$. Esta solução é usada em conjunto com a solução da equação homogênea da lei de conservação, para obtermos a solução numérica da lei de conservação não homogênea.

Vários exemplos destas rotinas e programas principais estão disponíveis em claw/applications. Podemos ver um exemplo simples em claw/clawpack/1d/example. A documentação completa pode ser encontrada no código fonte, em

claw/clawpack/1d/claw1/claw1.f.

Os comentários acima para o caso unidimensional aplicam-se em geral para duas e três dimensões também. Por exemplo, em duas dimensões é preciso fornecer dois resolvedores de Riemann, rpn2 e rpt2, um na direção normal à fronteira e a outra na direção transversal. Para uma discussão mais completa veja LeVeque [15] e o código pode ser encontrado em

claw/clawpack/2d/claw2/claw2.f.

A seguir, falaremos sobre como implementar as condições de fronteira empregadas no CLAWPACK.

\subsection{Condições de fronteira e Variáveis auxiliares}

Condições de fronteira - As condições de fronteira são fornecidas estendendo-se a malha com duas células em cada direção além da fronteira física. Estas células adicionais são chamadas muitas vezes "células-fantasmas" e são preenchidas no começo de cada passo de tempo de uma maneira que depende da natureza das condições de contorno da fronteira física. O fornecimento de dados sobre estas células nos permite resolver o problema de Riemann na fronteira mesma. Para o método de primeira ordem, ou de segunda ordem na ausência de limitadores, é suficiente calcular o valor atual em todas as células físicas. O segundo conjunto de células fantasma é necessário somente para a função limitadora, que considera as ondas nas células adjacentes sobre um lado ou no outro. 
No código CLAWPACK, o número de células-fantasmas em cada lado é determinado por um parâmetro $\mathrm{mbc}$, o qual normalmente assume o valor $\mathrm{de} \mathrm{mbc}=2$, mas pode ser trocado se um novo método é implementado com um stencil maior. O usuário deve especificar uma rotina de condições de fronteira bc1 em uma dimensão, ou bc2 em duas dimensões, a qual é chamada no início de cada passo de tempo para estender os dados às células-fantasmas. Muitos exemplos de diferentes tipos de condições de fronteira estão incluídas no CLAWPACK, tais como condições de fronteira periódicas, influxo especificado, e paredes sólidas para dinâmica de gases. Algumas condições de fronteira gerais podem ser encontradas em

$$
\begin{aligned}
& \text { claw/clawpack/1d/bc/bc1ex0.f - extrapolação } \\
& \text { claw/clawpack/1d/bc/bc1per.f - periódicas } \\
& \text { claw/clawpack/1d/bc/bc1inf.f - influxo para advecção } \\
& \text { claw/clawpack/1d/bc/bc1wall.f - paredes sólidas para Euler, acústica, }
\end{aligned}
$$

etc.

Em duas dimensões existem arquivos similares no diretório claw/clawpack/2d/bc.

Um conjunto de condições de fronteira que merece discussão especial são as obtidas por extrapolação, muitas vezes usada em fronteiras onde há escoamento para o exterior do domínio. Em muitos problemas o domínio físico deve ser truncado para um domínio computacional menor e é desejável que as ondas saindo do domínio computacional façam isso com um mínimo de reflexão na fronteira computacional. Existe uma grande quantidade de literatura sobre o desenvolvimento de condições de fronteira artificiais "absorventes" ou "não reflexivas", para esta situação. Para o Método de Propagação de Ondas descrito aqui, e portanto para os métodos tipo Godunov, é muitas vezes possível fazer um trabalho razoável em termos de fronteiras não reflexivas simplesmente extrapolando a solução do interior das células-fantasmas em cada passo de tempo, mesmo com extrapolação de "ordem zero" (isto é, constantes), na qual às célulasfantasmas são atribuídos os valores da primeira célula dentro do domínio.

Variáveis auxiliares - Nos códigos claw1 e claw2 existe um parâmetro aux que pode ser usado para armazenar variáveis auxiliares que são definidas sobre a mesma malha de $q$, mas que 
são parte da definição do problema. Exemplos incluem parâmetros físicos como densidade.

\subsection{Termos forçantes}

Em uma dimensão, a forma geral das equações com termo forçante que é resolvida com o CLAWPACK é:

$$
\kappa(x) q_{t}+A(q, x, t) q_{x}=\psi(q, \kappa, x, t) .
$$

Termos forçantes são tratados pelo CLAWPACK empregando um método de passo fracionário, no qual alternamos entre resolver a equação homogênea

$$
\kappa q_{t}+A q_{x}=0
$$

e a equação

$$
\kappa q_{t}=\psi .
$$

Usamos uma "divisão de Strang", Strang [29], o qual consiste em:

(a) Resolver a equação a equação (4.7) com a metade do passo de tempo $\Delta t / 2, \operatorname{logo}$

(b) Resolver a equação (4.6) com o passo total $\Delta t$, usando como dado o resultado obtido em (a) e, finalmente,

(c) Resolver, novamente, a equação (4.7) com o passo $\Delta t / 2$, usando como dado o resultado obtido em (b).

Pode parecer que temos avançado um passo de tempo de comprimento $2 \Delta t$, mas na verdade só temos avançado um passo $\Delta t$, pois em cada etapa só temos usado uma parte de nosso problema original (4.5). Podemos também inverter os papeis, isto é, resolvendo primeiro a equação (4.6). Isto tem a vantagem de dar resultados de precisão de segunda ordem para problemas com soluções suaves. Veja por exemplo LeVeque [18].

As rotinas do CLAWPACK permitem especificar um termo forçante fornecendo a função src1 (em uma dimensão) que resolve a equação (4.7). Isto tipicamente implica em resolvermos um sistema de equações diferenciais ordinárias em cada célula da malha, por qualquer método que o usuário escolha. Termos forçantes originam-se por exemplo ao reduzir leis de conservação 
multi-dimensionais para um problema de menor dimensão. Veja por exemplo LeVeque [15].

Termos forçantes rígidos - A aproximação pelo método de passo fracionário pode não ser apropriada para todos os problemas com termos forçantes. Em particular, problemas com termo forçante "rígido" (isto é, se sua solução numérica requer, talvez numa porção do intervalo de solução, reduzir significativamente o comprimento do passo para evitar instabilidade) devem ser tratados com muito cuidado. Usar um método de passo fracionário geralmente requer resolver escalas de tempo e espaço que podem requerer uma malha muito mais fina. Veja LeVeque-Yee [23] por exemplo.

Uma possível aproximação para tais problemas seria usar refinamento de malhas adaptativas, por exemplo usando o código do AMRCLAW [5]. Existem outros métodos que foram usados satisfatoriamente em combinação com refinamento adaptativo; veja Bourlioux [7] e LeVequeShyue [22]. Por outro lado, alguns problemas com termo forçante rígido podem ser tratados satisfatoriamente usando métodos de passo fracionário, como os discutidos por Pember [25].

Em geral devemos ter cuidado ao especificar as condições de fronteira quando usamos um método de passo fracionário; veja LeVeque [16]. Porém, para problemas típicos em que os termos forçantes são simplesmente equações diferenciais ordinárias em cada célula da malha, parece adequado fazer o seguinte em cada passo de tempo:

- Primeiro estenda $q_{i}$ para as células-fantasmas usando a rotina bc que implementa as condições de fronteira.

- Resolva a equação (4.7) com passo de tempo $\Delta t / 2$ em todas as células, incluindo as célulasfantasmas.

- Tome um passo de tempo $\Delta t$ sobre a parte hiperbólica (4.6), com todos os pontos interiores atuais (sem as células-fantasmas).

- Resolva a equação (4.7) com passo de tempo $\Delta t / 2$ em todas as células. Os novos valores nas células-fantasmas podem não ser significativos, mas estas são sobrescritas pela rotina bc no começo do passo seguinte de tempo. Estas não precisam ser atualizadas neste passo, mas por simplicidade elas são, pois a mesma rotina src1 é usada em ambos passos. 
Nas rotinas CLAWPACK, bc é chamada antes do primeiro chamado de src1, portanto esta é implementada naturalmente. O ponto importante para o usuário é que as células-fantasmas também serão atualizadas ao menos na primeira chamada de src1 para manter a consistência com o resto da solução. 


\section{Resultados Numéricos}

Neste capítulo, mostraremos os resultados numéricos obtidos ao resolvermos a equação

$$
q_{t}+\left(\frac{1}{n} q^{n}\right)_{x}=q^{m}
$$

utilizando o Método de Propagação de Ondas implementado no CLAWPACK. Resolveremos esta equação para vários dados iniciais e para $n$ e $m$, inteiros positivos.

A presença do termo forçante na equação (5.1) pode fazer com que a solução não seja limitada num tempo finito para algum ponto $\bar{x}$, isto é,

$$
\lim _{\substack{x \rightarrow x \\ t \rightarrow \bar{t}}}|q(x, t)|=+\infty
$$

onde $|\bar{x}|<\infty$ e $\bar{t}<\infty$. Denominaremos este comportamento como explosão em tempo finito (ou simplesmente explosão) e o ponto $(\bar{x}, \bar{t})$ como ponto de explosão.

A solução da equação escalar (5.1) pode vir a apresentar ou a formação de um choque ou explodir em algum tempo finito, ainda que o dado inicial seja suave. Não é claro o interrelacionamento destes eventos e, como veremos a seguir por intermédio de exemplos, poderá ocorrer apenas a formação de um choque, de uma explosão ou de um choque sucedido por uma explosão. Utilizando o CLAWPACK, investigaremos numericamente o que ocorre com a solução de (5.1) para alguns valores de $m$ e de $n$, inteiros positivos, considerando os seguintes dados iniciais, mostrados na Figura (5.1) abaixo. 


$$
\begin{aligned}
& q_{0}(x)=\sigma=1, \\
& q_{0}(x)=\frac{1}{1+e^{-x}}, \\
& q_{0}(x)= \begin{cases}1-0.25 x^{2}, & -2<x<2 \\
0 \quad, & x \leq-2 \text { ou } x \geq 2,\end{cases} \\
& q_{0}(x)= \begin{cases}-2 \cos \left(\frac{\pi}{4}(x-2)\right) & 0<x<4 \\
0 \quad, & x \leq 0 \text { ou } x \geq 4,\end{cases} \\
& q_{0}(x)=0.5+\sin (2 \pi x) .
\end{aligned}
$$

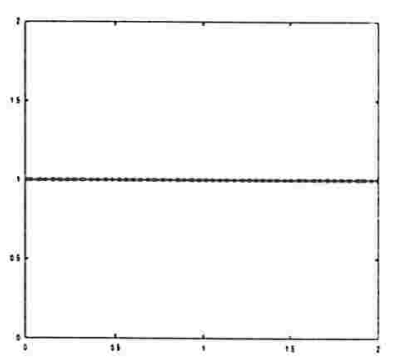

(a)

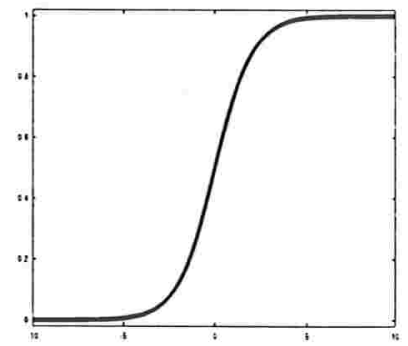

(b)

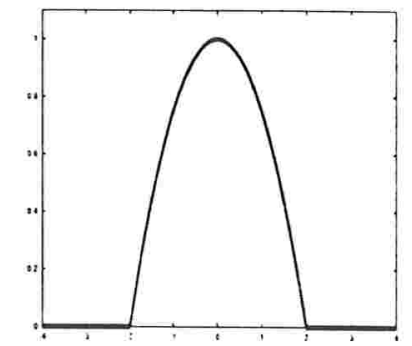

(c)

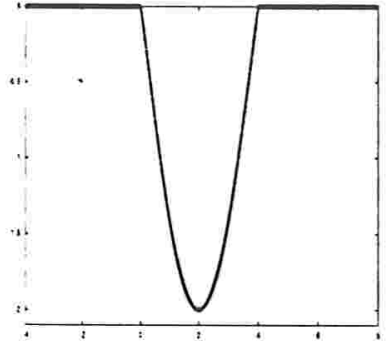

(d)

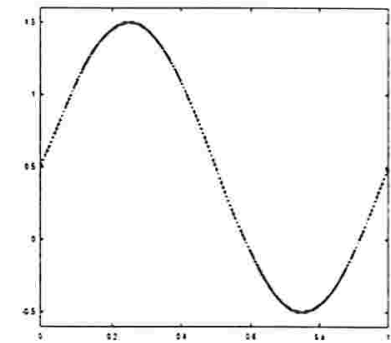

(e)

Figura 5.1: (a) Dado inicial (5.2), (b) Dado inicial (5.3), (c) Dado inicial (5.4), (d) Dado inicial (5.5), (e) Dado inicial (5.6). 
Nas seções a seguir, mostraremos os resultados numéricos obtidos para a equação (5.1) os quais serão agrupados em três casos: $n=m, n<m$ e $n>m$.

\subsection{Caso $\mathrm{n}=\mathrm{m}$}

Nas tabelas a seguir, apresentaremos os valores obtidos numericamente para os dados iniciais (5.2)-(5.6), vistos anteriormente. Na primeira coluna temos a condição inicial, "c.i.", considerada. $O$ instante da formação do choque e sua localização serão denotados por $t_{c}$ e $x_{c}$, respectivamente, e o instante de explosão e sua localização por $t_{e}$ e $x_{e}$. Utilizaremos o simbolo "\#" para denotarmos a inexistência e "?" a incerteza de um determinado valor.

Tabela 5.1: Instantes de tempo e posições dos choques $\left(x_{c}, t_{c}\right)$ e/ou das explosões $\left(x_{e}, t_{e}\right)$.

\begin{tabular}{||c||c|c||c|c||}
\hline \hline \multicolumn{5}{|c||}{$\mathrm{n}=\mathrm{m}=2$} \\
\hline \hline c.i. & $t_{c}$ & $x_{c}$ & $t_{e}$ & $x_{e}$ \\
\hline 5.2 & $\nexists$ & $\nexists$ & 1.0020 & $\mathbb{R}$ \\
\hline 5.3 & $\nexists$ & $\nexists$ & $1+3 \times 10^{-7}$ & $+\infty$ \\
\hline 5.4 & 0.7975 & 1.9025 & 1.0644 & 27.8350 \\
\hline 5.5 & 0.6450 & 0.0050 & $\nexists$ & $\nexists$ \\
\hline 5.6 & 0.1475 & 0.5736 & 1.2503 & $?$ \\
\hline \hline
\end{tabular}

Tabela 5.2: Instantes de tempo e posições dos choques $\left(x_{c}, t_{c}\right)$ e/ou das explosões $\left(x_{e}, t_{e}\right)$.

\begin{tabular}{||c||c|c||c|c||}
\hline \hline \multicolumn{5}{||c||}{$\mathrm{n}=\mathrm{m}=3$} \\
\hline \hline c.i. & $t_{c}$ & $x_{c}$ & $t_{e}$ & $x_{e}$ \\
\hline 5.2 & $\nexists$ & $\nexists$ & 0.5000 & $\mathbb{R}$ \\
\hline 5.3 & $\nexists$ & $\nexists$ & $0.5+5 \times 10^{-9}$ & $+\infty$ \\
\hline 5.4 & 0.4520 & 1.2950 & 0.5289 & 18.7350 \\
\hline 5.5 & 0.1105 & 3.2025 & 0.1336 & 20.2800 \\
\hline 5.6 & 0.0749 & 0.4962 & 0.5819 & $?$ \\
\hline \hline
\end{tabular}


Tabela 5.3: Instantes de tempo e posições dos choques $\left(x_{c}, t_{c}\right)$ e/ou das explosões $\left(x_{e}, t_{e}\right)$.

\begin{tabular}{||l||c|c||c|c||}
\hline \hline \multicolumn{5}{||c||}{$\mathrm{n}=\mathrm{m}=4$} \\
\hline \hline c.i. & $t_{c}$ & $x_{c}$ & $t_{e}$ & $x_{e}$ \\
\hline 5.2 & $\nexists$ & $\nexists$ & 0.3333 & $\mathbb{R}$ \\
\hline 5.3 & $\nexists$ & $\nexists$ & 0.3333 & $+\infty$ \\
\hline 5.4 & 0.3075 & 0.9650 & 0.3498 & 13.4000 \\
\hline 5.5 & 0.8050 & 0.2575 & $\nexists$ & $\nexists$ \\
\hline 5.6 & 0.0420 & 0.4525 & 0.3030 & $?$ \\
\hline \hline
\end{tabular}

Tabela 5.4: Instantes de tempo e posições dos choques $\left(x_{c}, t_{c}\right)$ e/ou das explosões $\left(x_{e}, t_{e}\right)$.

\begin{tabular}{||c||c|c||c|c||}
\hline \hline \multicolumn{5}{||c||}{$\mathrm{n}=\mathrm{m}=5$} \\
\hline \hline c.i. & $t_{c}$ & $x_{c}$ & $t_{e}$ & $x_{e}$ \\
\hline 5.2 & $\nexists$ & $\nexists$ & 0.2500 & $\mathbb{R}$ \\
\hline 5.3 & $\nexists$ & $\nexists$ & 0.2500 & $+\infty$ \\
\hline 5.4 & 0.2365 & 0.8000 & 0.2605 & 11.1500 \\
\hline 5.5 & 0.0145 & 2.7425 & 0.0165 & 13.2300 \\
\hline 5.6 & 0.0230 & 0.4250 & 0.1602 & $?$ \\
\hline \hline
\end{tabular}

Para os valores escolhidos de $m=n$, o comportamento das soluções para os dados iniciais (5.5), com $m$ ímpar, (5.4) e (5.6) apresenta qualitativamente as mesmas características, sendo que ocorre a formação de um choque o qual é sucedido por uma explosão. Para o dado inicial (5.5), com $m$ par, ocorre apenas a formação de um choque. Para os dados iniciais (5.2) e (5.3) observamos ainda um outro comportamento: a solução só explode.

No que se segue, por simplicidade, incluiremos apenas os gráficos obtidos para o caso $n=$ $m=2$. Quando utilizamos as condições iniciais (5.2) e (5.3), obtivemos os conjuntos de gráficos mostrados pelas Figuras 5.1 e 5.2, a seguir. 


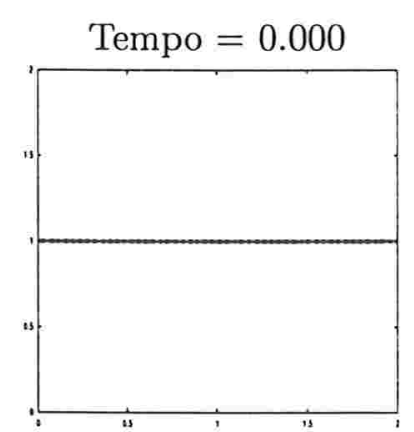

(a)

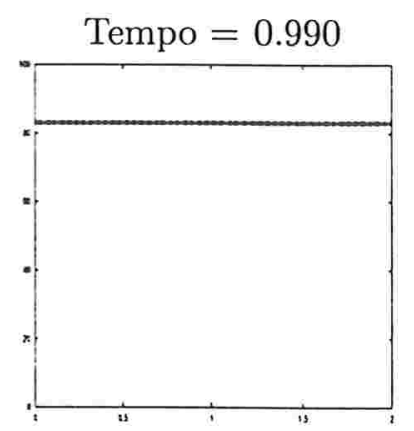

(b)

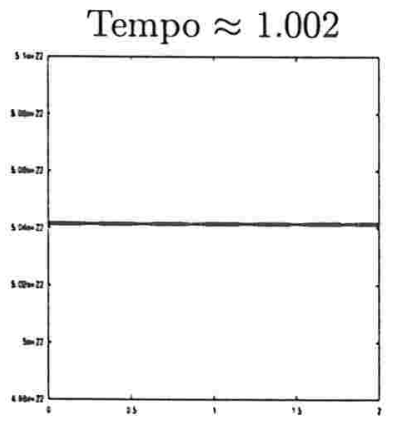

(c)

Figura 5.2: $m=n=2$. (a) Dado inicial (5.2). (c) Explosão da solução.

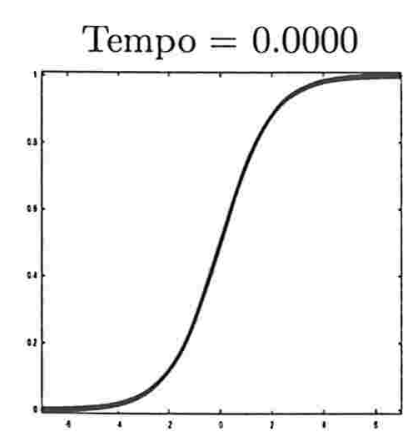

(a)

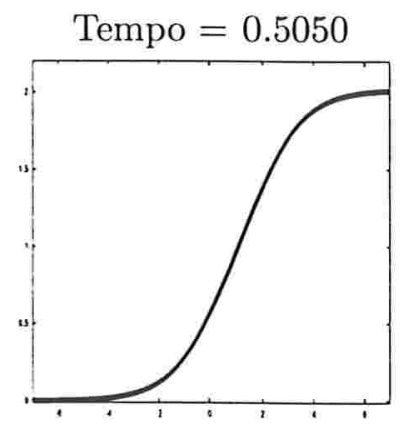

(b)

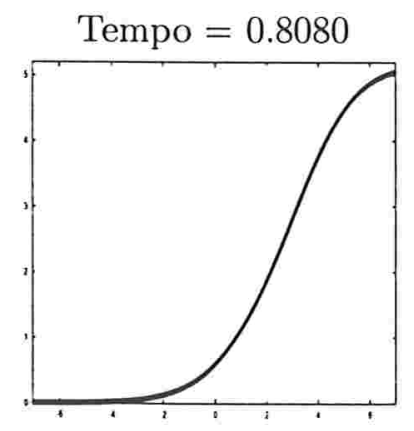

(c)

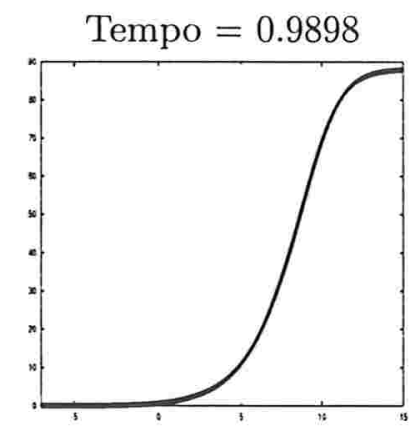

(d)

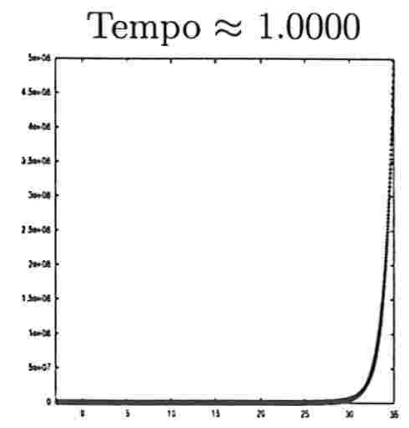

(e)

Figura 5.3: $m=n=2$. (a) Dado inicial 5.3. (e) Explosão da solução. 
Para as condições (5.2) e (5.3), a explosão da solução da equação (5.1), mostrada nos gráficos anteriores, ocorre também para os outros valores tabelados de $m, n=m \neq 1$, nunca havendo a formação de choques. Isto faz sentido pois, se usarmos o método das características para analisarmos a solução clássica da equação (5.1), obteremos

$$
q(x, t)=\frac{q_{0}(\lambda)}{\left[1-(m-1) t q_{0}^{m-1}(\lambda)\right]^{1 /(m-1)}} \quad, \quad m>1,
$$

onde $\lambda$ é dado por

$$
x=\lambda-\frac{1}{m-1} \ln \left[1-(m-1) t q_{0}^{m-1}(\lambda)\right] .
$$

De (5.7), vemos que a solução explodirá no tempo

$$
T_{e x}=\min _{\lambda}\left\{\frac{1}{(m-1) q_{0}^{m-1}(\lambda)}\right\}
$$

para $m>1$, e $q_{0}>0$, como ocorre nos dados iniciais (5.2) e (5.3). De (5.9), vemos que a solução explodirá também quando $m>1$, ímpar, e $q_{0}<0$, como ocorre com o dado inicial (5.5). Finalmente, quando $m$ for par e $q_{0}<0$ a solução nunca explodirá (veja as Tabelas 5.1 e 5.3 e a Figura 5.5).

Se o dado inicial for constante, $q_{0}(x)=\sigma>0$, como em (5.2) onde $\sigma=1$, então a solução explodirá no tempo

$$
T_{e x}=\frac{1}{(m-1) \sigma^{m-1}} .
$$

Da expresão acima, quando $m=2$ e $\sigma=1$, o instante previsto teoricamente para a explosão é $T_{e x}=1$; da Tabela 5.1, observamos que o instante de tempo onde ocorre a explosão, obtido numericamente, é $t_{e}=1.0020$, aproximando-se bastante do instante obtido teoricamente.

Para o caso $n=m=1$, a equação (5.1) toma a forma $q_{t}+q_{x}=q$, cuja solução é dada por

$$
q(x, t)=q_{0}(x-t) e^{t}
$$

de onde vemos que a solução só "explodirá" no infinito para os dados iniciais dados.

A análise teórica desenvolvida acima pode ser extendida para o estudo do instante de formação de choques quando o dado inicial for diferenciável. Para obtermos o instante de formação 
do choque calcularemos a derivada em $x$ de (5.7),

$$
q_{x}=\frac{q_{0}^{\prime}(\lambda)}{\left[1-(m-1) t q_{0}^{m-1}(\lambda)\right]^{\frac{1}{m-1}}\left[1-(m-1) t q_{0}^{m-2}(\lambda)\left(q_{0}(\lambda)-q_{0}^{\prime}(\lambda)\right)\right]} .
$$

Igualando o denominador de (5.10) a zero, obtemos

$$
T_{1}=\frac{1}{\sup _{\lambda}\left\{(m-1) q_{0}^{m-1}(\lambda)\right\}}
$$

para $\lambda$ tal que $q_{0}^{m-1}(\lambda)>0, \mathrm{e}$

$$
T_{2}=\frac{1}{\sup _{\lambda}\left\{(m-1) q_{0}^{m-2}(\lambda)\left(q_{0}(\lambda)-q_{0}^{\prime}(\lambda)\right)\right\}},
$$

para $\lambda$ tal que $q_{0}^{m-2}(\lambda)\left(q_{0}(\lambda)-q_{0}^{\prime}(\lambda)\right)>0$, onde $T_{1}$ é o instante de explosão dado por (5.9) e $T_{2}$ o instante de formação do choque $\left(T_{c h}=T_{2}\right)$, se $T_{2}<T_{1}$.

Note que, para que $T_{c h}$ tenha sentido, além da diferenciabilidade de $q_{0}$ precisaremos impor que $q_{0}^{m-2}\left(q_{0}-q_{0}^{\prime}\right)$ seja limitado superiormente.

Com base nas considerações acima, vemos que a condição inicial (5.3) realmente não pode vir a desenvolver choque pois se houvesse a formação de choque teríamos $T_{2}<T_{1}$ e, portanto, $q_{0}^{\prime}<0$. Entretanto, temos $q_{0}^{\prime}>0, \forall \lambda \in \mathbb{R}$.

Estas mesmas considerações nos servem de base para calcularmos o instante de tempo de formação do choque para o dado inicial (5.6). Para $m=n=2$ teremos que

$$
T_{c h}=\frac{1}{\max _{\lambda}\{0.5+\sin (2 \pi \lambda)-2 \pi \cos (2 \pi \lambda)\}}, \text { para } 0<\lambda<1,
$$

e, portanto,

$$
T_{c h}=0.14742336 \text {. }
$$

O instante de tempo teórico acima, no qual ocorre a formação do choque, aproxima-se bastante do valor obtido numericamente, $t_{c}=0.1475$ (veja a Tabela 5.1). Para o dado inical (5.6), para todos os valores de $m=n$ empregados, apesar de termos podido determinar aproximadamente o instante de explosão $t_{e}$, não pudemos determinar o ponto exato onde ela ocorre, $x_{e}$, pois a solução se desloca abruptamente dificultando a análise (veja por exemplo a Figura 5.6 (g)-(h)). 
Observe que a análise para a formação de choque apresentada presupõe alguma regularidade da condição inicial, não podendo ser aplicada para as condições iniciais (5.4) e (5.5), as quais desenvolvem choques (Figuras 5.4 e 5.5, respetivamente). Note também que este tipo de análise não se presta ao cálculo do instante de explosão da solução quando este é posterior à formação do choque, isto é, (5.9) não pode ser utilizada uma vez que, após do choque, a solução deixa de ser suave. Assim sendo, podemos apenas aproximar numericamente o instante de explosão para as condições iniciais (5.4) e (5.6) (Figuras 5.4 e 5.6, respetivamente). 


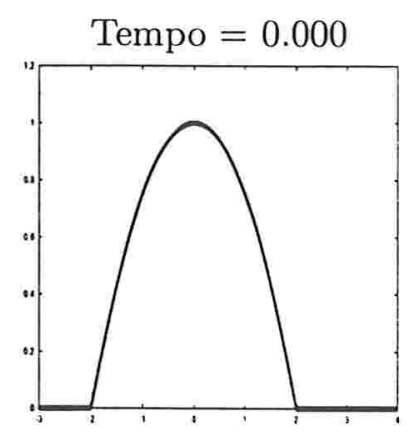

(a)

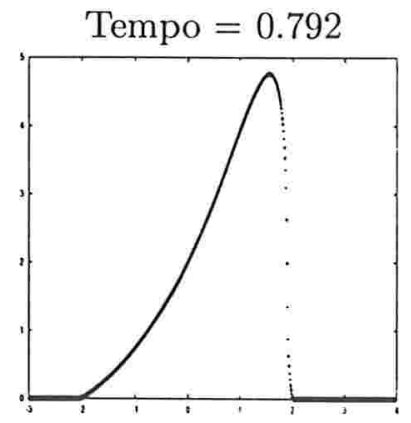

(d)

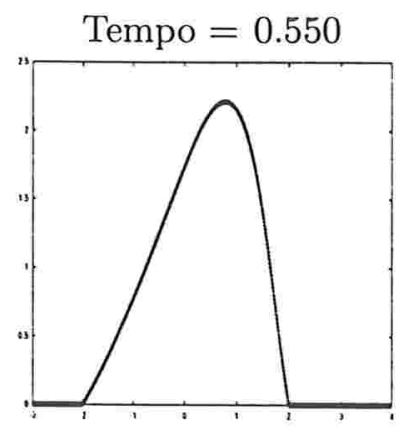

(b)

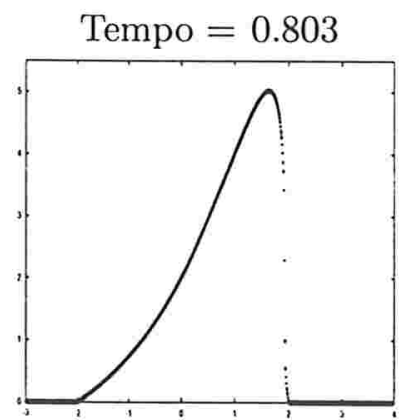

(e)

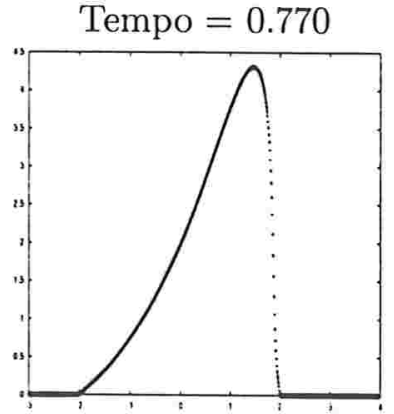

(c)

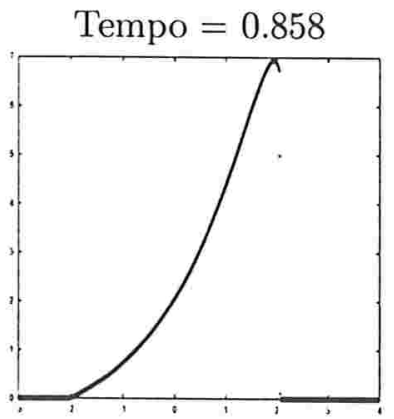

(f)

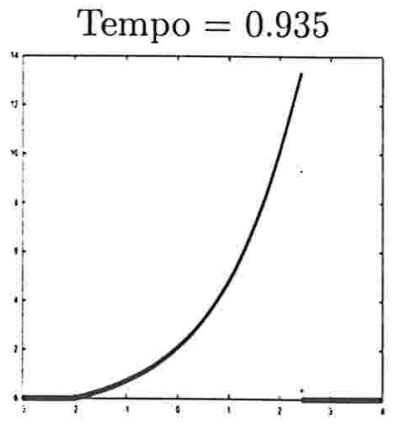

(g)

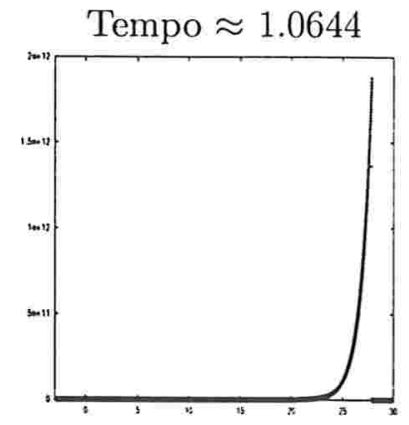

(h)

Figura 5.4: $m=n=2$. (a) Dado inicial 5.4. (d)-(e) Formação do choque. (h) Explosão da solução. 


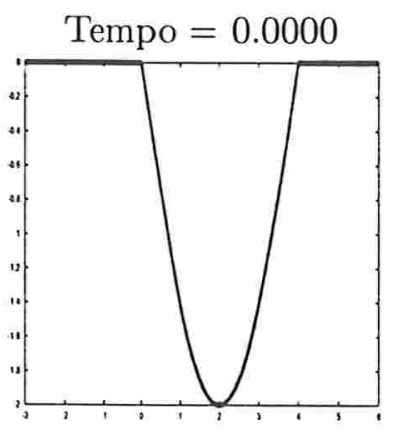

(a)

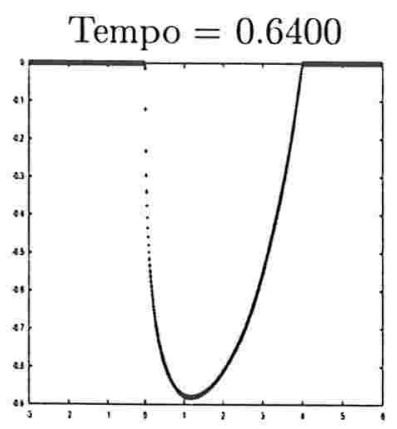

(d)

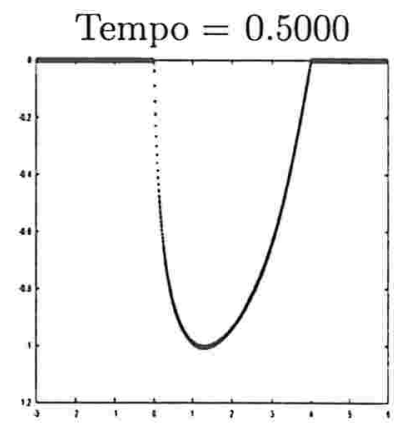

(b)

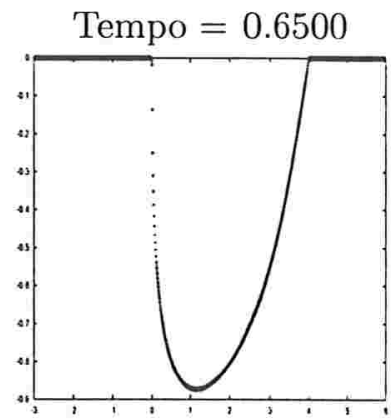

(e)

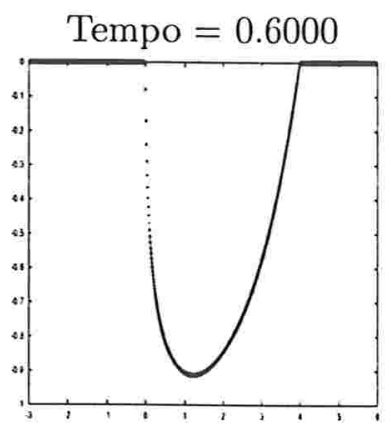

(c)

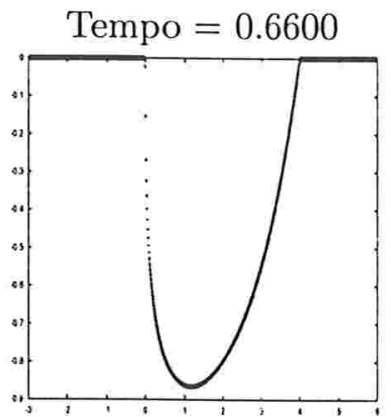

(f)

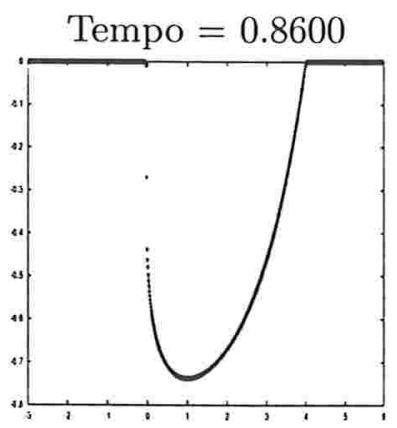

(g)

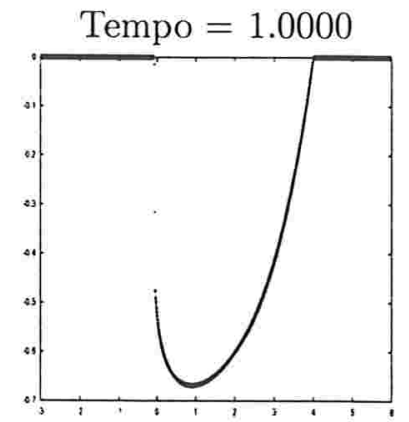

(h)

Figura 5.5: $m=n=2$. (a) Dado inicial 5.5. (d)-(e) Formação do choque. 


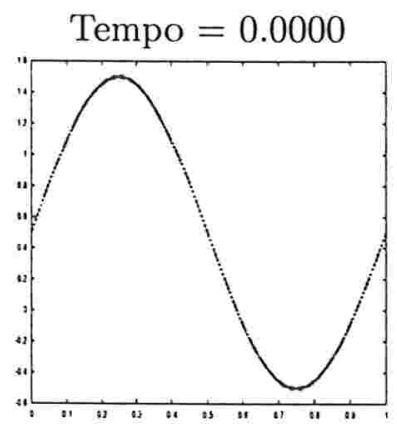

(a)

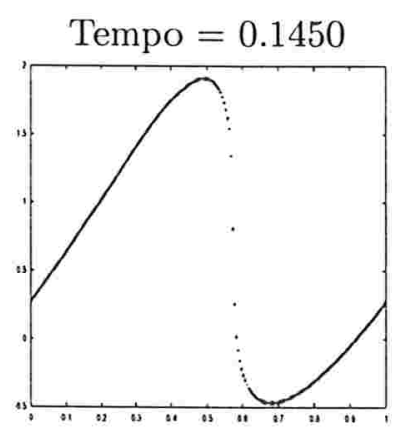

(d)

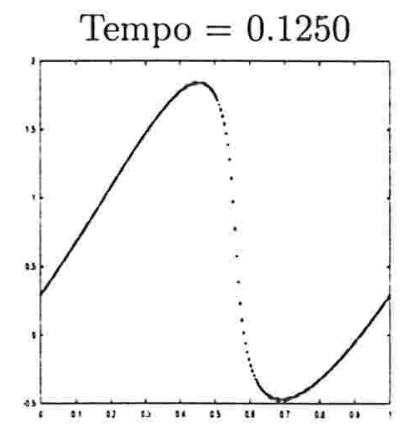

(b)

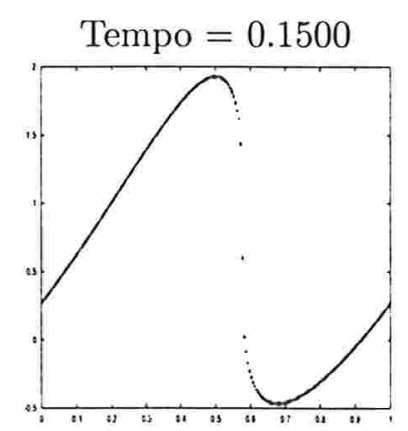

(e)

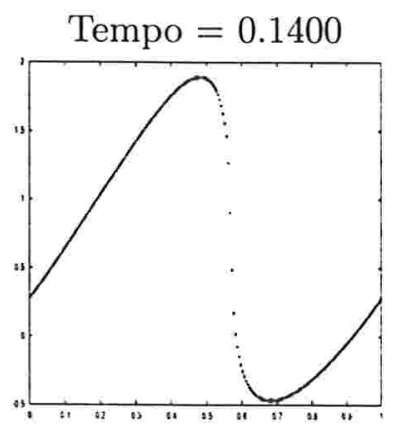

(c)

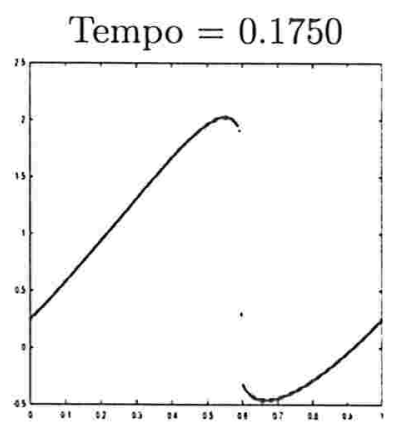

(f)

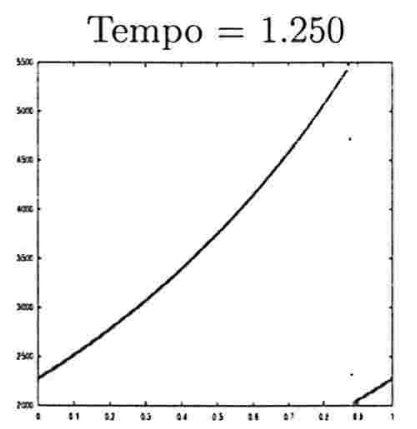

(g)

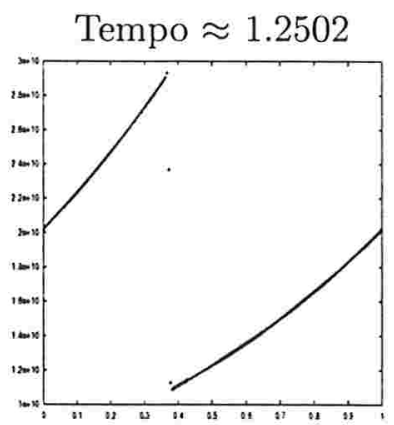

(h)

Figura 5.6: $m=n=2$. (a) Dado inicial 5.6. (d)-(e) Formação do choque. (h) Explosão da solução. 
Com base nos resultados obtidos, finalizamos esta seção com a seguinte conjectura:

1. Quando $q_{0}$ é não decrescente e assume valores positivos em alguma região de seu domínio de definição, teremos apenas uma explosão.

2. Quando $q_{0}$, a condição inicial, é decrescente em alguma região de seu domínio de definição haverá a formação de um choque, o qual é sucedido por uma explosão, exceto quando $q_{0}<0$ e $m$ é par.

3. Quando $q_{0}<0$ e $m$ é par, teremos a formação de um choque sem explosão.

\subsection{Caso $\mathrm{n}>\mathrm{m}$}

Nesta seção, apresentaremos os resultados obtidos para os dados iniciais (5.2)-(5.6), definidos no início deste capítulo. A solução para o dado inicial (5.2), $q_{0}(x)=\sigma>0, x \in \mathbb{R}$, como vimos na seção anterior, independe do valor de $n$ e é dada por

$$
q(x, t)=\frac{\sigma}{\left[1-(m-1) t \sigma^{m-1}\right]^{1 /(m-1)}},
$$

ocorrendo apenas uma explosão no tempo

$$
T_{e x}=\frac{1}{(m-1) \sigma^{m-1}} .
$$

Assim sendo, nas Tabelas 5.5, 5.6 e 5.7, abaixo, ele não será incluído.

Tabela 5.5: Instantes de tempo e posições dos choques $\left(x_{c}, t_{c}\right)$ e/ou das explosões $\left(x_{e}, t_{e}\right)$.

\begin{tabular}{||c||c|c||c|c||}
\hline \hline \multicolumn{5}{||c||}{$\mathrm{n}=3, \mathrm{~m}=2$} \\
\hline \hline c.i. & $t_{c}$ & $x_{c}$ & $t_{e}$ & $x_{e}$ \\
\hline 5.3 & $\nexists$ & $\nexists$ & $?$ & $?$ \\
\hline 5.4 & 0.5625 & 1.5555 & $?$ & $?$ \\
\hline 5.5 & 0.9950 & 3.8300 & $\nexists$ & $\nexists$ \\
\hline 5.6 & 0.0852 & 0.5085 & $?$ & $?$ \\
\hline \hline
\end{tabular}


Tabela 5.6: Instantes de tempo e posições dos choques $\left(x_{c}, t_{c}\right)$ e/ou das explosões $\left(x_{e}, t_{e}\right)$.

\begin{tabular}{||c||c|c||c|c||}
\hline \hline \multicolumn{5}{||c||}{$\mathrm{n}=5, \mathrm{~m}=3$} \\
\hline \hline c.i. & $t_{c}$ & $x_{c}$ & $t_{e}$ & $x_{e}$ \\
\hline 5.3 & $\nexists$ & $\nexists$ & $?$ & $?$ \\
\hline 5.4 & 0.3375 & 0.8100 & $?$ & $?$ \\
\hline 5.5 & 0.0405 & 3.1413 & $?$ & $?$ \\
\hline 5.6 & 0.0300 & 0.4425 & $?$ & $?$ \\
\hline \hline
\end{tabular}

Tabela 5.7: Instantes de tempo e posições dos choques $\left(x_{c}, t_{c}\right)$ e/ou das explosões $\left(x_{e}, t_{e}\right)$.

\begin{tabular}{||c||c|c||c|c||}
\hline \hline \multicolumn{5}{||c||}{$\mathrm{n}=6, \mathrm{~m}=4$} \\
\hline \hline c.i. & $t_{c}$ & $x_{c}$ & $t_{e}$ & $x_{e}$ \\
\hline 5.3 & $\nexists$ & $\nexists$ & $?$ & $?$ \\
\hline 5.4 & 0.2625 & 0.9750 & $?$ & $?$ \\
\hline 5.5 & 0.0850 & 0.7125 & $\nexists$ & $\nexists$ \\
\hline 5.6 & 0.0200 & 0.4275 & $?$ & $?$ \\
\hline \hline
\end{tabular}


Para o caso $n>m$, o desenvolvimento de uma análise teórica mais geral como a apresentada na seção anterior fica substancialmente mais complexo e, portanto, nós nos restringiremos apenas àquelas conclusões obtidas anteriormente que puderem ser aqui aplicadas, além dos resultados obtidos numericamente.

Inicialmente, observamos que para o dado (5.3) não ocorre a formação de choque. Além disso, é difícil julgar numericamente se há ou não uma explosão num tempo finito. O comportamento das soluções observado para os valores escolhidos de $m$ e $n$, para os dados iniciais (5.4), (5.5) (para $m$ ímpar) e (5.6), apresenta qualitativamente as mesmas características, havendo a formação de choque, sendo, uma vez mais, difícil julgar numericamente se ocorre ou não uma explosão em tempo finito. Entretanto, para a condição inicial (5.5) com $m$ par podemos afirmar que após o choque que se forma não ocorre explosão. A justificativa para este fato pode ser encontrada se observarmos a equação (5.9) e lembrarmos que, para esta condição inicial $q_{0}(\lambda) \leq 0, \lambda \in \mathbb{R}$. Por simplicidade, incluiremos a seguir apenas os gráficos obtidos para o caso $n=3$ e $m=2$. 


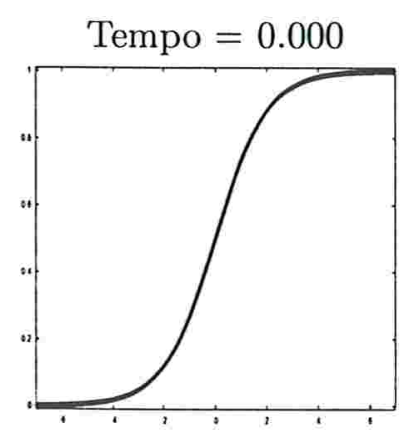

(a)

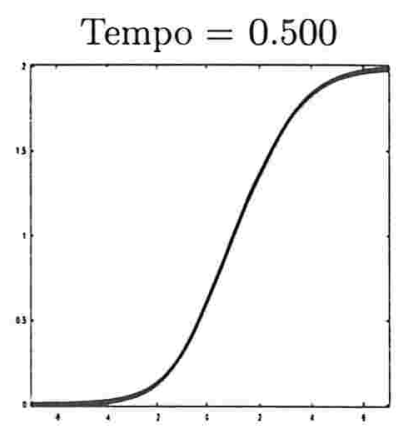

(b)

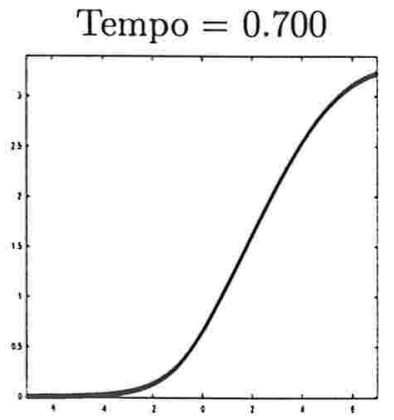

(c)

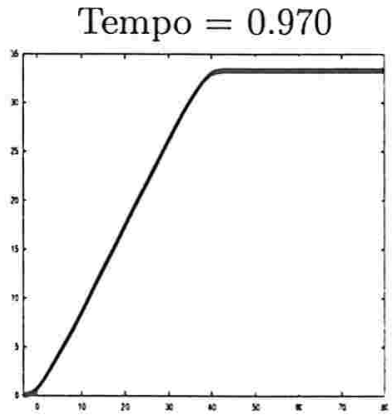

(d)

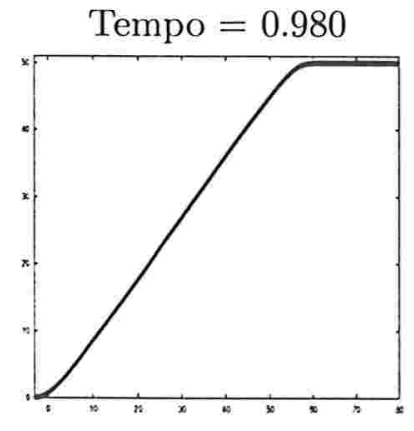

(e)

Figura 5.7: $n=3, m=2$. (a)-Dado inicial (5.3). (b)-(e)-Continuação da solução sem a formação de choque. 


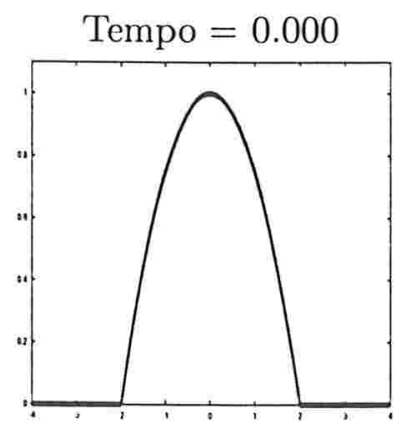

(a)

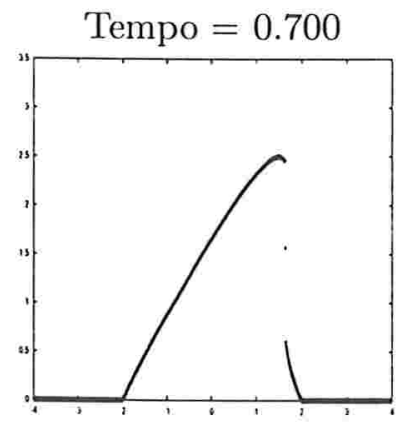

(d)

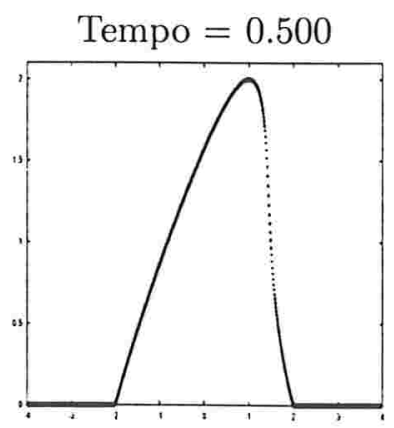

(b)

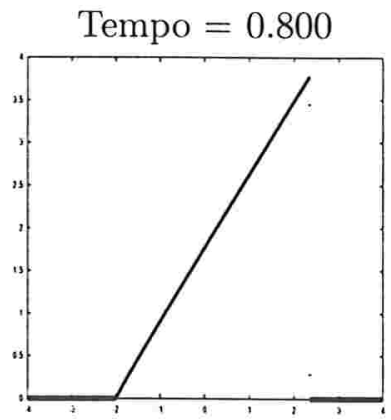

(e)

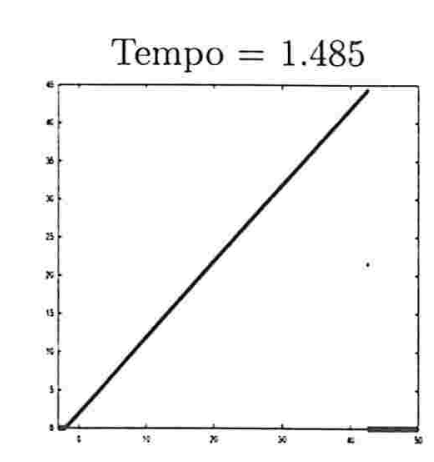

(g)

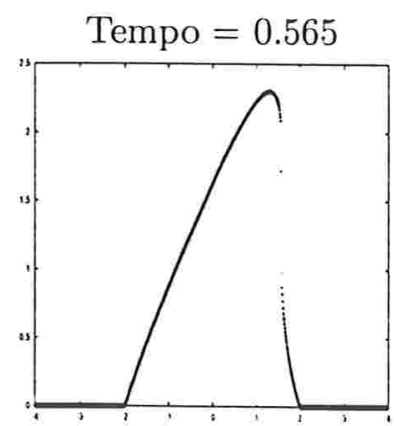

(c)

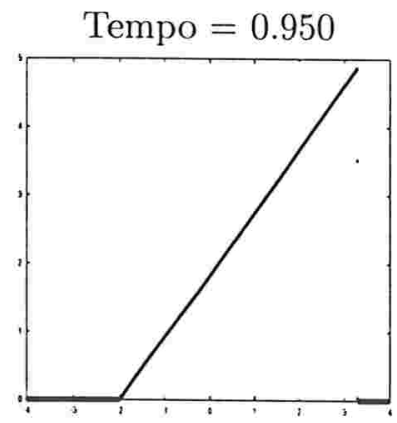

(f)

Figura 5.8: $n=3, m=2$. (a)-Dado inicial (5.4). (c)-Formação do choque. 


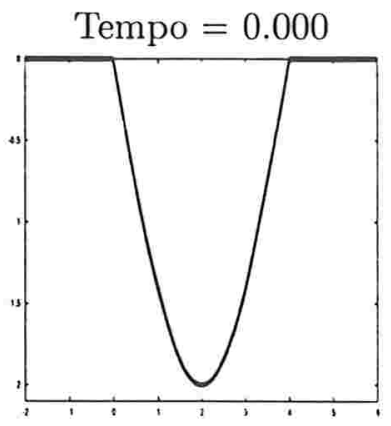

(a)

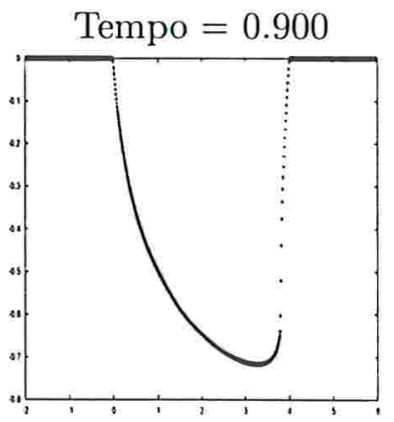

(d)

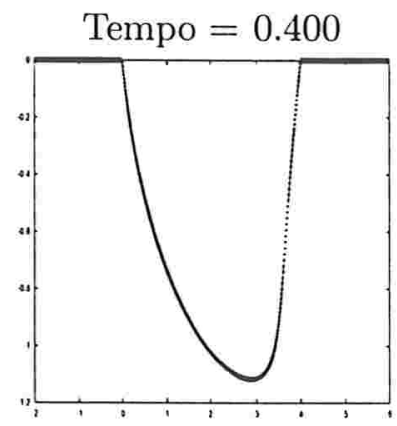

(b)

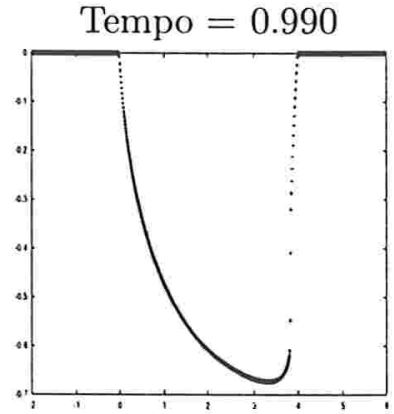

(e)

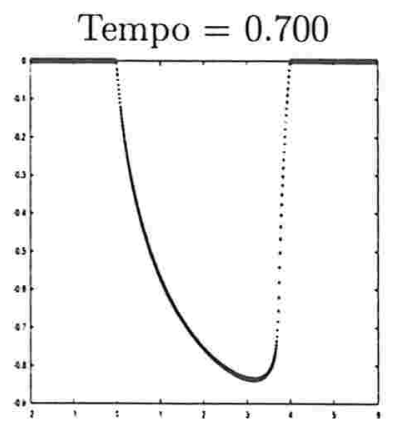

(c)

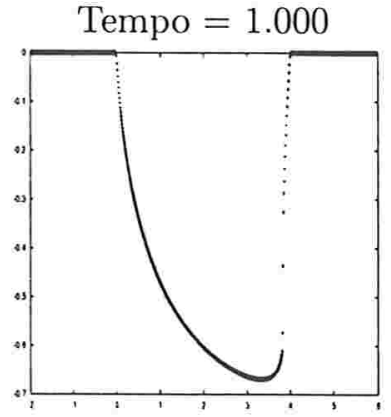

(f)

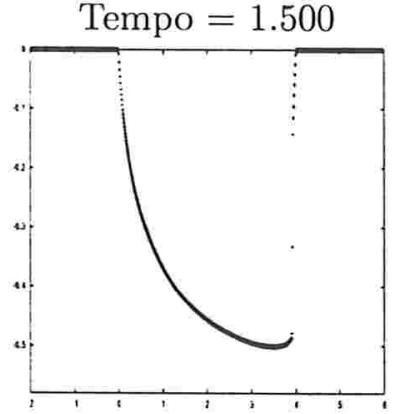

(g)

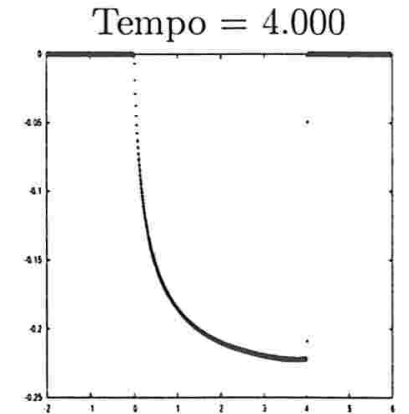

(h)

Figura 5.9: $n=3, m=2$. (a)-Dado inicial (5.5). (d)-(e)-Formação do choque. 


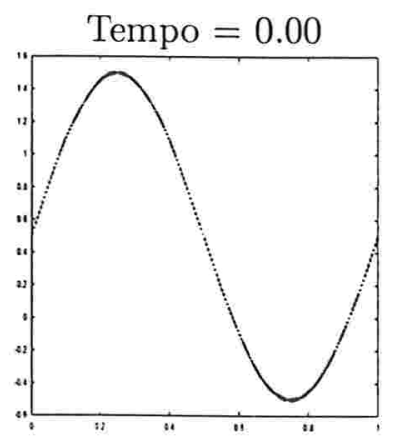

(a)

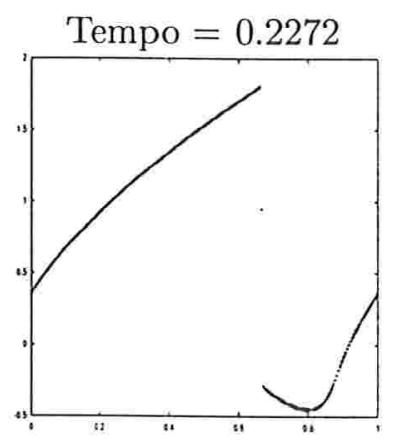

(d)

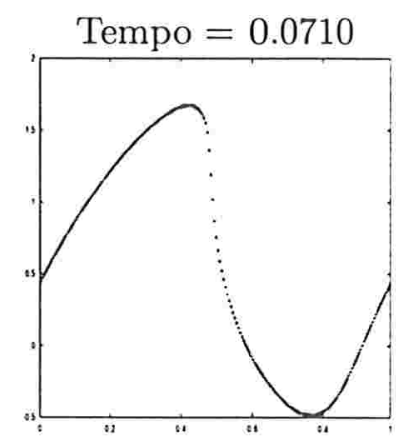

(b)

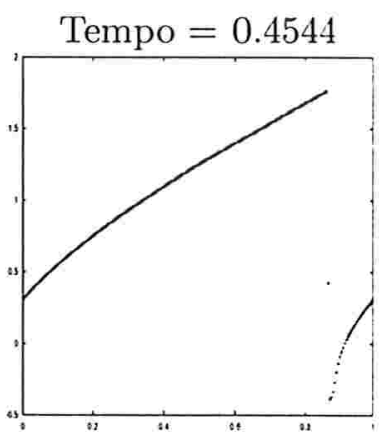

(e)

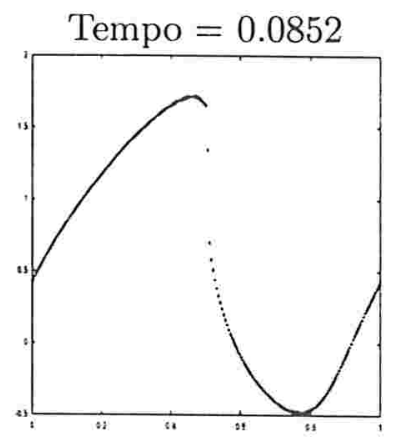

(c)

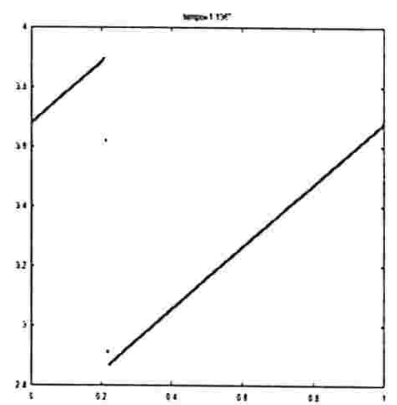

(f)

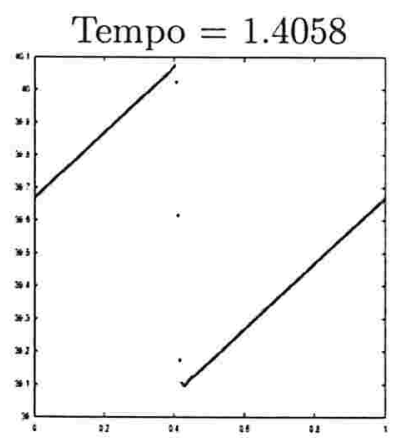

(g)

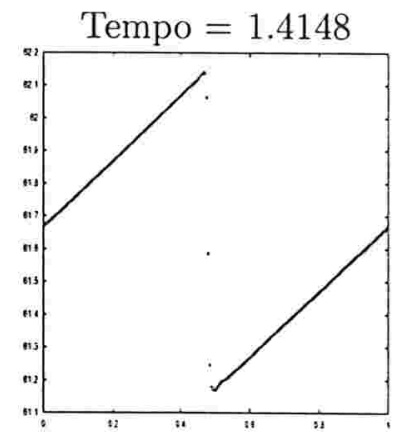

(h)

Figura 5.10: $n=3, m=2$.(a)-Dado inicial (5.6). (b)-(c)-Formação do choque. 
Com base nos resultados numéricos obtidos para a equação (5.1), conjecturamos que:

1. Quando $q_{0}>0$ e $q_{0}^{\prime}>0$, teremos apenas explosão da solução (sem choque.)

2. A solução desenvolve um choque quando $q_{0}^{\prime}<0$ em alguma região do domínio.

Finalizamos esta seção observando que quando há a formação de um choque, este ocorre cada vez mais cedo conforme $n$ aumenta. Numericamente não pudemos decidir, para a maior parte dos dados iniciais, se a solução permanece limitada ou se ela explode pois, por mais que tenhamos ampliado o domínio de integração numérica, a solução cresce mas não atinge uma ordem de grandeza que nos permita afirmar que ela, de fato, explodiu. Por outro lado, também não podemos afirmar que ela fica efetivamente limitada (não explode) pois a solução cresce.

\subsection{Caso $\mathrm{n}<\mathrm{m}$}

Nas tabelas a seguir, apresentaremos os valores obtidos numericamente para os dados iniciais (5.3)-(5.6). Uma vez mais, com base nas considerações anteriores, a solução para o dado inicial (5.2) será omitida desta seção, vindo sempre a desenvolver uma explosão de acordo com (5.9).

$\mathrm{Na}$ análise que se segue, será de grande ajuda considerarmos os dois novos dados iniciais:

$$
\begin{aligned}
& q_{0}(x)= \begin{cases}\frac{1}{4}\left(1-0.25 x^{2}\right), & -2<x<2 \\
0, & x \leq-2 \text { ou } x \geq 2,\end{cases} \\
& q_{0}(x)= \begin{cases}5\left(1-0.25 x^{2}\right) & , \quad-2<x<2 \\
0 & , \quad x \leq-2 \text { ou } x \geq 2 .\end{cases}
\end{aligned}
$$

Acrecentamos os resultados relativos aos dados iniciais (5.11) e (5.12) nas Tabelas 5.8 e 5.9, abaixo, para ilustrar que o comportamento da solução obtido para o dado inicial (5.4) é muito diferente dos obtidos para os dados iniciais (5.11) e (5.12), apesar destes diferirem apenas por uma constante multiplicativa. 
Tabela 5.8: Instantes de tempo e posições dos choques $\left(x_{c}, t_{c}\right)$ e/ou das explosões $\left(x_{e}, t_{e}\right)$.

\begin{tabular}{||c||c|c||c|c||}
\hline \hline \multicolumn{5}{||c||}{$\mathrm{n}=2, \mathrm{~m}=4$} \\
\hline \hline c.i. & $t_{c}$ & $x_{c}$ & $t_{e}$ & $x_{e}$ \\
\hline 5.3 & $\nexists$ & $\nexists$ & 0.333333 & $+\infty$ \\
\hline 5.4 & $\nexists$ & $\nexists$ & 0.33429 & 0.5050 \\
\hline 5.5 & 0.610 & 0.0125 & $\nexists$ & $\nexists$ \\
\hline 5.6 & $\nexists$ & $\nexists$ & 0.09994 & 0.4750 \\
\hline 5.11 & 4.1500 & 1.9900 & $\nexists$ & $\nexists$ \\
\hline 5.12 & $\nexists$ & $\nexists$ & 0.00266 & 0.0150 \\
\hline \hline
\end{tabular}

Tabela 5.9: Instantes de tempo e posições dos choques $\left(x_{c}, t_{c}\right)$ e/ou das explosōes $\left(x_{\boldsymbol{e}}, t_{\boldsymbol{e}}\right)$.

\begin{tabular}{||c||c|c||c|c||}
\hline \hline \multicolumn{5}{||c||}{$\mathrm{n}=5, \mathrm{~m}=6$} \\
\hline \hline c.i. & $t_{c}$ & $x_{c}$ & $t_{e}$ & $x_{e}$ \\
\hline 5.3 & $\nexists$ & $\nexists$ & $?$ & $?$ \\
\hline 5.4 & 0.1984 & 0.6425 & $?$ & $?$ \\
\hline 5.5 & 0.69 & 3.6800 & $\nexists$ & $\nexists$ \\
\hline 5.6 & 0.01795 & 0.4100 & $?$ & $?$ \\
\hline 5.12 & $\nexists$ & $\nexists$ & $6.474 \mathrm{e}-05$ & 0.1650 \\
\hline \hline
\end{tabular}

O comportamento das soluções observado para os valores escolhidos de $m$ e $n$ não apresentam qualitativamente as mesmas características para cada um dos dados iniciais considerados. Um exemplo marcante deste fato é obtido quando empregamos os dados inicias (5.4), (5.11) e (5.12), os quais diferem entre si apenas por uma constante multiplicativa (1, 1/4 e 5 , respetivamente). No caso $n=2$ e $m=4$, Tabela $5.8,(5.4)$ e (5.12) exibem o mesmo comportamento vindo a explodir num tempo finito, ao passo que (5.11) desenvolve apenas um choque! Para o mesmo conjunto de dados, quando $n=5$ e $m=6$, ocorre uma explosão para o dado (5.12), desta vez, havendo a formação de um choque para (5.4) e (5.11). Não há a semelhança de comportamento esperada para estes dados nem para um mesmo par de valores de $n$ e $m$, nem para pares diferentes destes valores. 
Durante a simulação numérica, para alguns valores da Tabela 5.9, foi difícil decidir se há ou não explosão pois, por mais que tenhamos ampliado nosso domínio de integração numérica, a solução cresce mas não atinge uma ordem de grandeza que nos leve a afirmar que ela explode. Assim mesmo, não podemos afirmar que ela fica limitada (não explode) pois a solução cresce.

Os tempos de explosão que pudemos, efetivamente, obter numericamente contidos nas Tabelas 5.8 e 5.9, concordam com os tempos previstos teoricamente, obtidos a partir da solução teórica,

$$
q(x, t)=\frac{q_{0}(\lambda)}{\left[1-(m-1) t q_{0}^{m-1}(\lambda)\right]^{1 /(m-1)}} \quad, \quad m>1,
$$

onde $\lambda$ é dado por

$$
x=\lambda-\frac{1}{n-m} q_{0}^{n-m}(\lambda)\left\{1-\left[1-(m-1) t q_{0}^{m-1}(\lambda)\right]^{(m-n) /(m-1)}\right\} .
$$

De (5.13), temos que o tempo de explosão será

$$
T_{e x}=\min _{\lambda}\left\{\frac{1}{(m-1) q_{0}^{m-1}(\lambda)}\right\}
$$

Para ilustrar o caso $n<m$, incluiremos a seguir os gráficos obtidos para $n=2$ e $m=4$. 


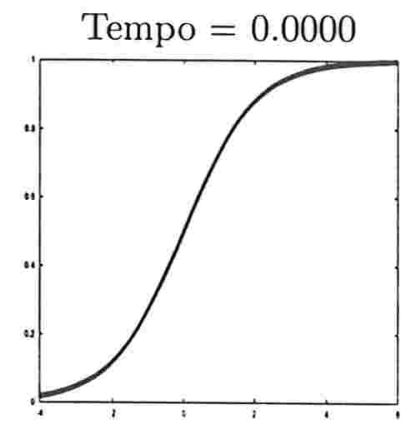

(a)

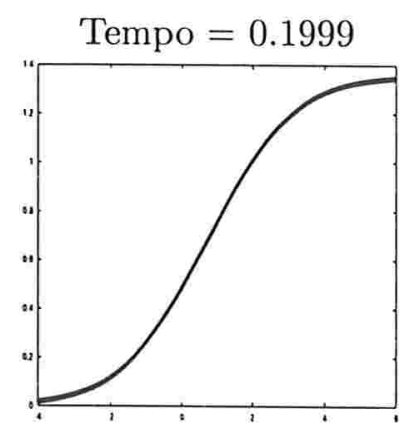

(d)

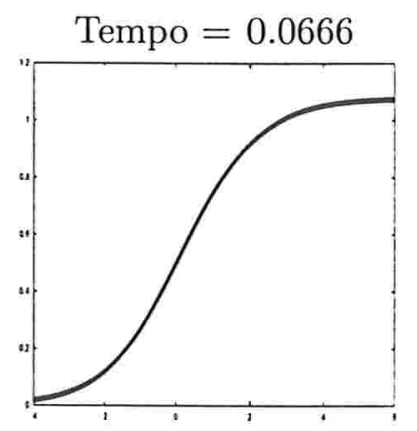

(b)

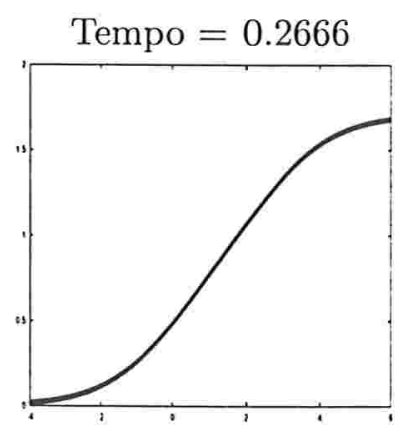

(e)

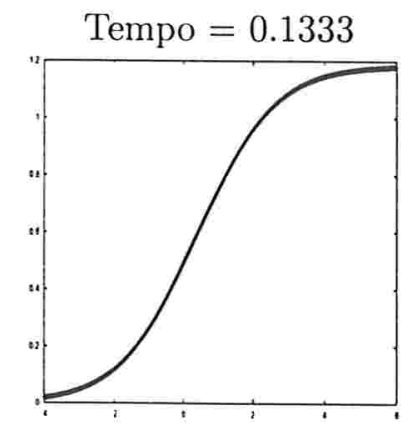

(c)

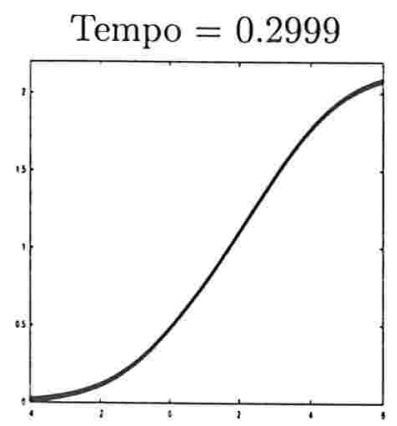

(f)

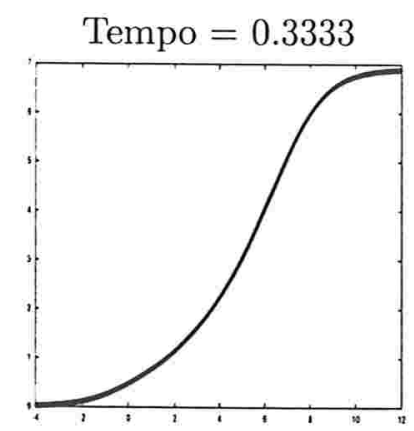

(g)

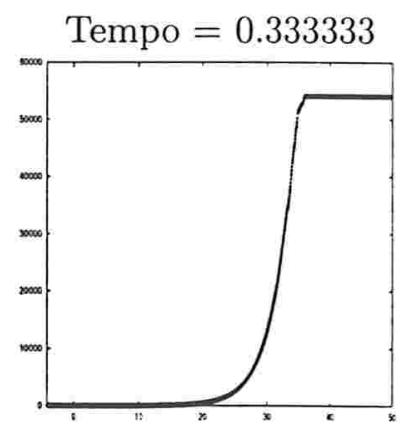

(h)

Figura 5.11: $n=2$ e $m=4$. (a)-Dado inicial (5.3). (i)-Explosão da solução. 


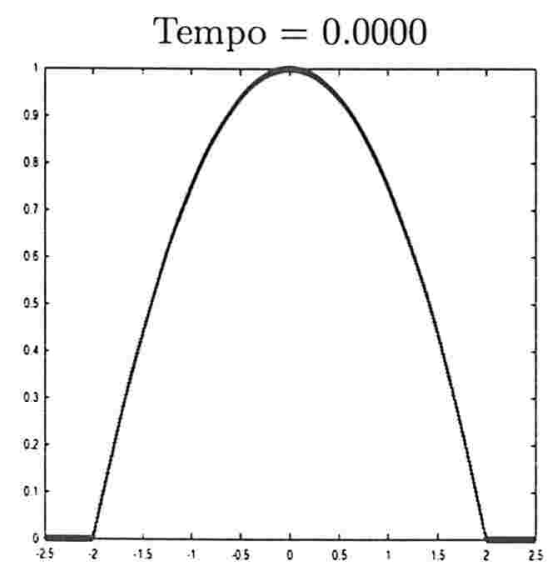

(a)

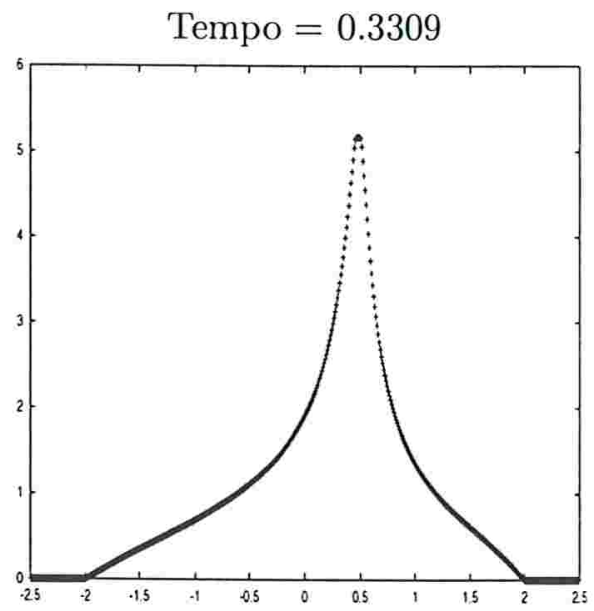

(c)

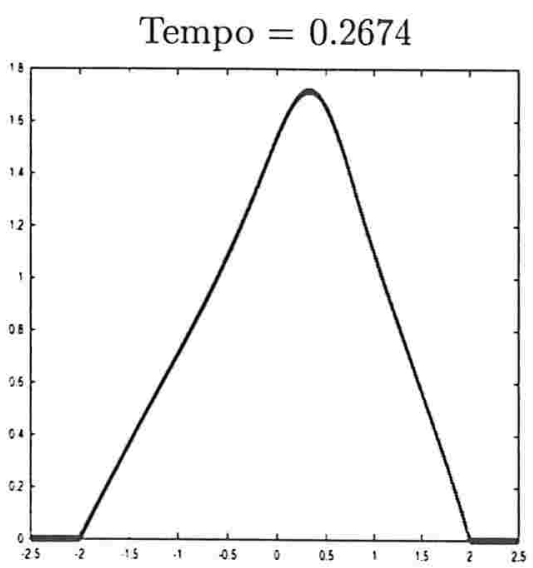

(b)

Tempo $\approx 0.33429$

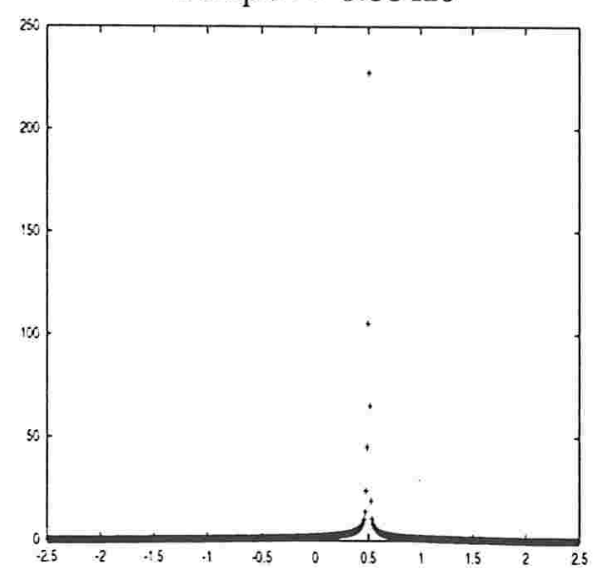

(d)

Figura 5.12: $n=2$ e $m=4$. (a)-Dado inicial (5.4). (d)-Explosão da solução. 


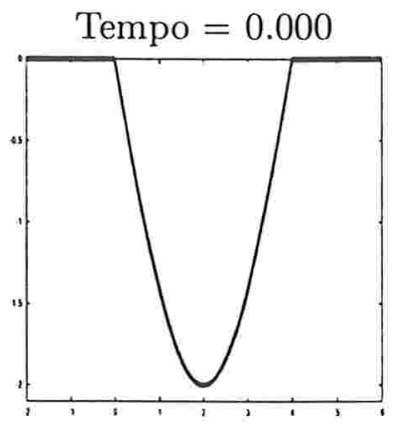

(a)

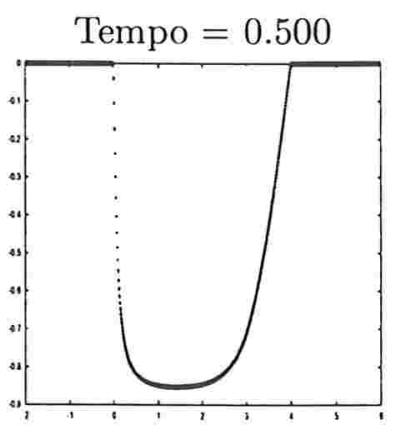

(d)

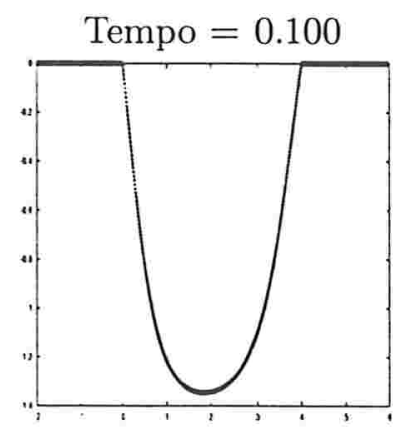

(b)

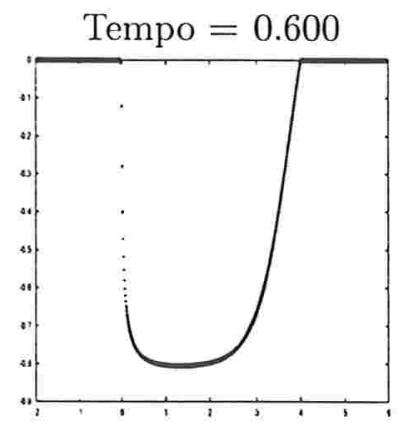

(e)

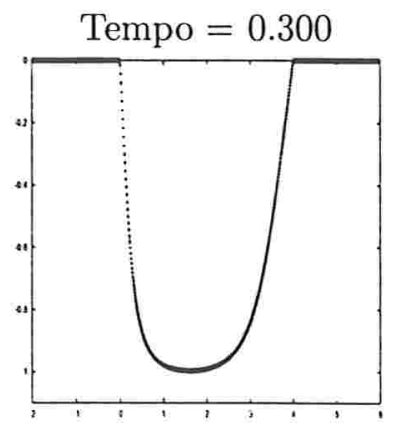

(c)

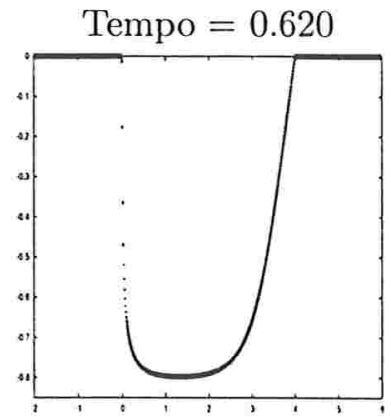

(f)

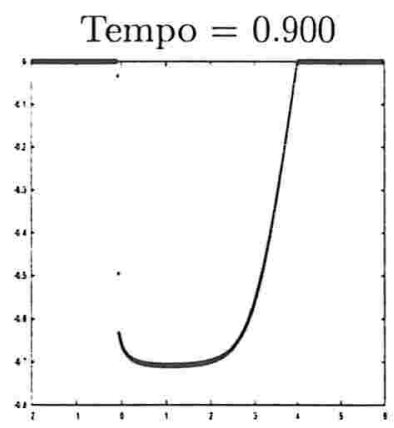

(g)

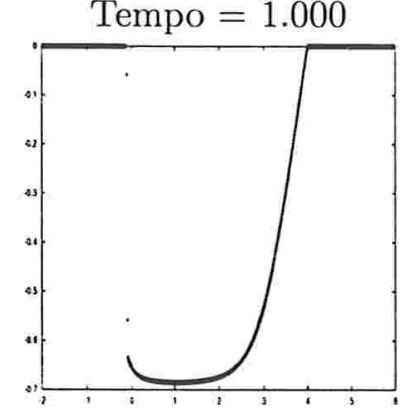

(h)

Figura 5.13: $n=2$ e $m=4$. (a)-Dado inicial (5.5). (e)-(f)-Formação do choque. 


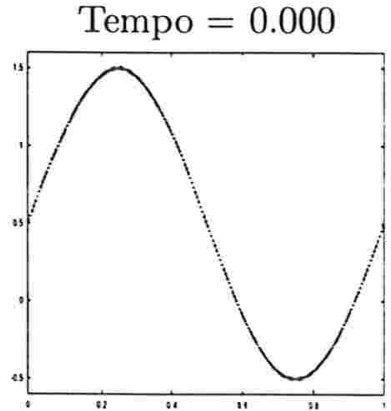

(a)

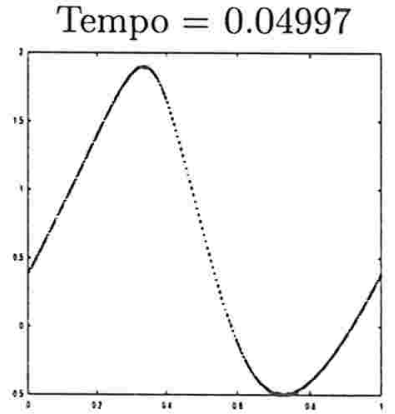

(b)

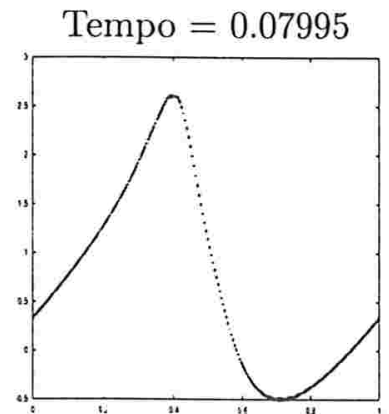

(c)

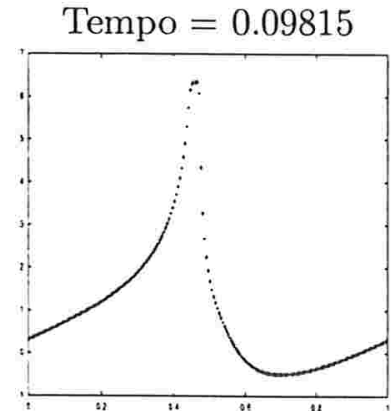

(d)

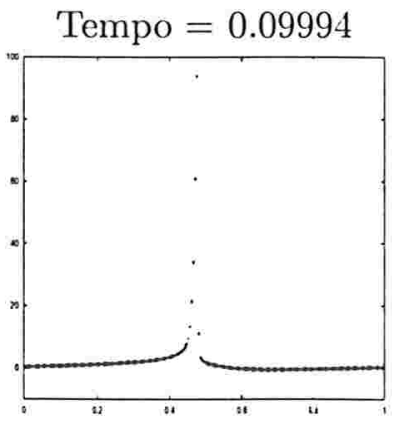

(e)

Figura 5.14: $n=2$ e $m=4$. (a)-Dado inicial (5.6). (e)-Explosão da solução. 


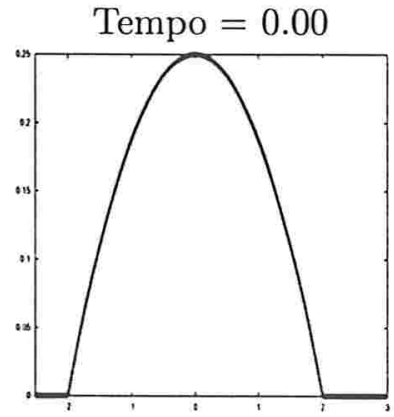

(a)

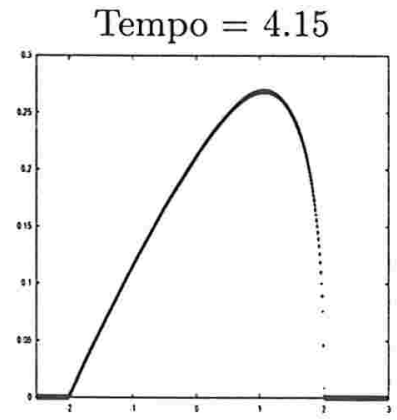

(d)

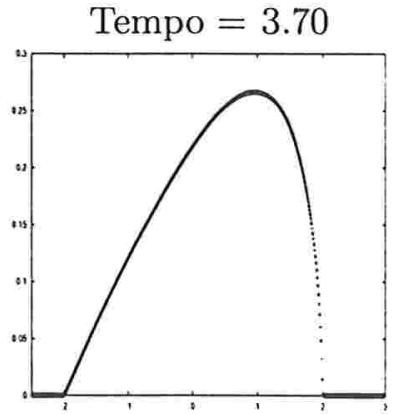

(b)

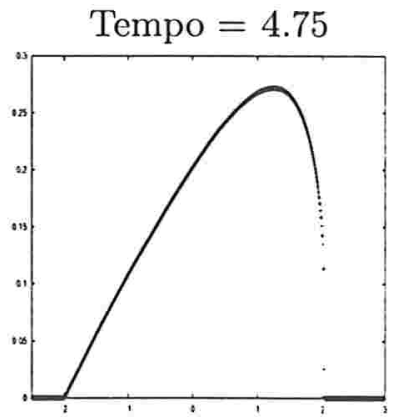

(e)

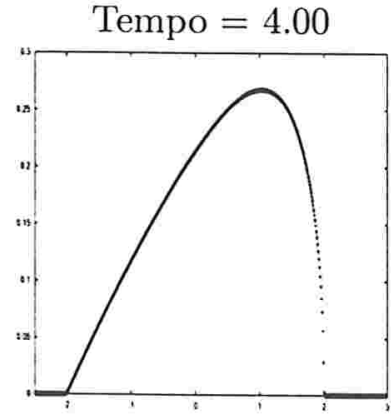

(c)

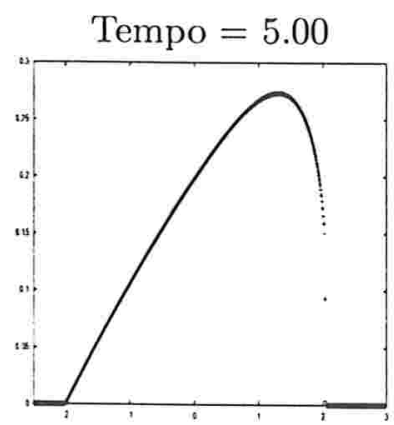

(f)

Figura 5.15: $n=2$ e $m=4$. (a)-Dado inicial (5.11). (b)-(e)-Formação do choque. 


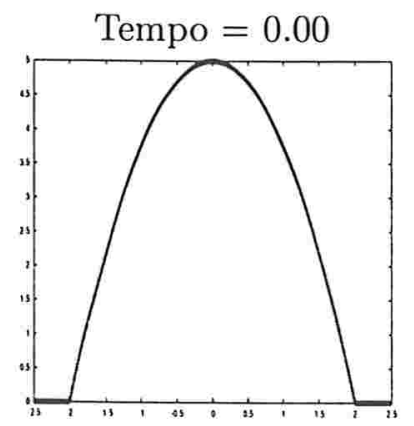

(a)

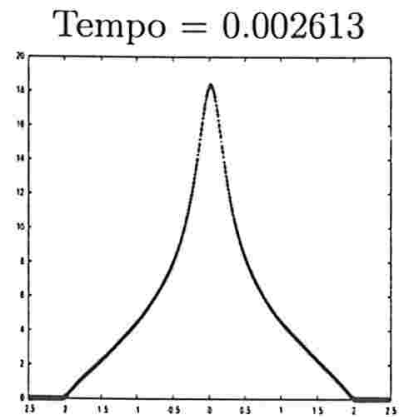

(d)

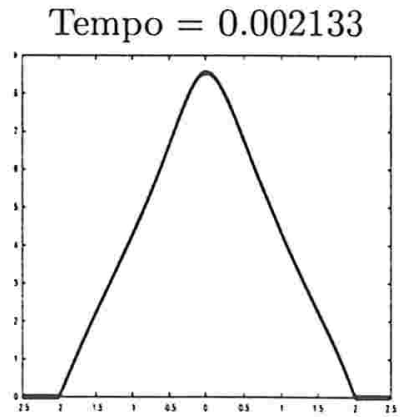

(b)

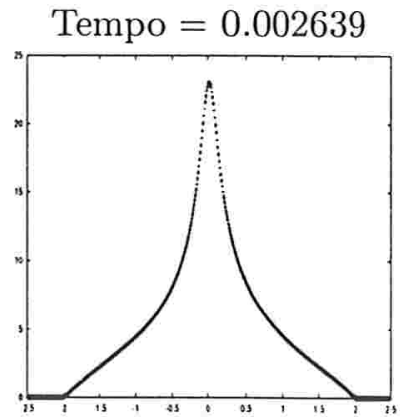

(e)

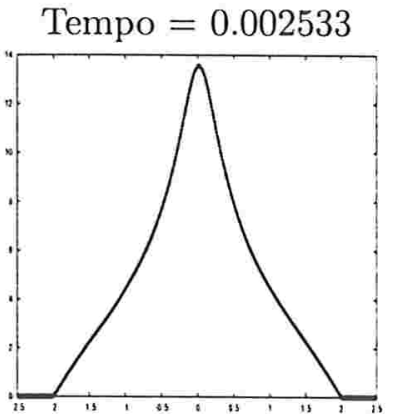

(c)

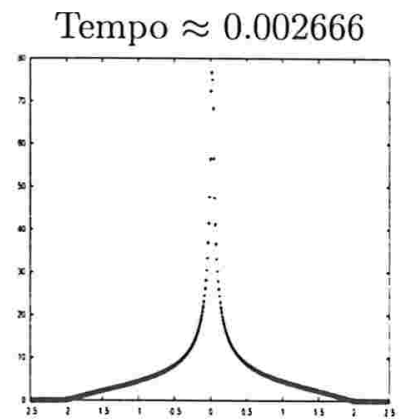

(f)

Figura 5.16: $n=2$ e $m=4$. (a)-Dado inicial (5.12). (f)-Explosão da solução. 
Finalizamos esta seção concluindo que, à vista dos resultados numéricos obtidos, não é possível conjecturar a existência de qualquer tipo de comportamento semelhante para os diversos dados utilizados. 


\section{Conclusões}

Neste trabalho, apresentamos a teoria básica sobre leis de conservação hiperbólicas escalares e o Método de Propagação de Ondas. Introduzido por LeVeque [19], [17], [20], [21], este método baseia-se na resolução de problemas de Riemann, sendo um método de alta resolução especialmente útil na simulação numérica de leis de conservação hiperbólicas.

LeVeque e colaboradores implementaram computacionalmente o Método de Propagação de Ondas num software denominado CLAWPACK (Conservation Laws Package). Aqui, explicamos onde encontrar, como baixar e como instalar o CLAWPACK.

A metodologia apresentada, por intermédio do CLAWPACK, foi utilizada para resolver a lei de conservação hiperbólica escalar com termo forçante,

$$
q_{t}+\left(\frac{1}{n} q^{n}\right)_{x}=q^{m}
$$

Dos resultados numéricos obtidos, pudemos extrair as seguintes conjecturas :

- Caso $n=m$ :

1. Quando $q_{0}$ é não decrescente e assume valores positivos em alguma região de seu dominio de definição, teremos apenas uma explosão.

2. Quando $q_{0}$, a condição inicial, é decrescente em alguma região de seu domínio de definição haverá a formação de um choque, o qual é sucedido por uma explosão, exceto quando $q_{0}<0$ e $m$ é par. 
3. Quando $q_{0}<0, m$ é par, teremos a formação de um choque, sem explosão.

- Caso $n>m$ :

1. Quando $q_{0}>0$ e $q_{0}^{\prime}>0$, teremos apenas explosão da solução.

2. A solução desenvolve um choque quando $q_{0}^{\prime}<0$ em alguma região do domínio.

Quando $n<m$, considerando os dados iniciais utilizados, não pudemos fazer conjectura alguma pois, por exemplo, para as condições (5.4) e (5.12) os resultados numéricos da Tabela 5.8 não apresentam resultados similares, apesar deles serem iguais exeto por uma constante multiplicativa. O mesmo acontece para outro par de valores de $n$ e $m$, como na Tabela 5.9. 


\section{Apêndice A}

\section{A.1 O Método de Godunov}

O Método de Godunov está baseado em resolver problemas de Riemann conforme avançamos no tempo. Soluções de problemas de Riemann são relativamente fáceis de calcular, dão informação substâncial acerca da estrutura característica e se prestam para o uso em métodos conservativos, uma vez que elas mesmas são soluções exatas de leis de conservação.

Para construir o método usamos a solução numérica $q_{i}^{n}$ para definir uma função constante por partes $\tilde{q}^{n}\left(x, t_{n}\right)$ com valor $q_{i}^{n}$ sobre a célula-malha $x_{i-1 / 2}<x<x_{i+1 / 2}$. Usamos $\tilde{q}^{n}\left(x, t_{n}\right)$ como dado inicial para a lei de conservação, que agora resolvemos extamente para obter $\tilde{q}^{n}(x, t)$ para $t_{n} \leq t \leq t_{n+1}$. A solução pode ser resolvida exatamente sobre um curto intervalo de tempo pois o dado inicial $\tilde{q}^{n}\left(x, t_{n}\right)$ é constante por partes e, portanto, define uma seqüência de problemas de Riemann. A solução exata é obtida simplesmente juntando os pedaços destas soluções de Riemann. Ver Fig.A.1.

Depois de obter esta solução sobre o intervalo $\left[t_{n}, t_{n+1}\right]$, definimos a solução aproximada $q_{i}^{n+1}$ em tempo $t_{n+1}$, tirando a média desta solução exata no tempo $t_{n+1}$,

$$
q_{i}^{n+1}=\frac{1}{h} \int_{x_{i-1 / 2}}^{x_{i+1 / 2}} \tilde{q}^{n}\left(x, t_{n+1}\right) d x .
$$

Estes valores são usados para definir novos dados constantes por partes $\tilde{q}^{n+1}\left(x, t_{n+1}\right)$ e o processo repete-se. 


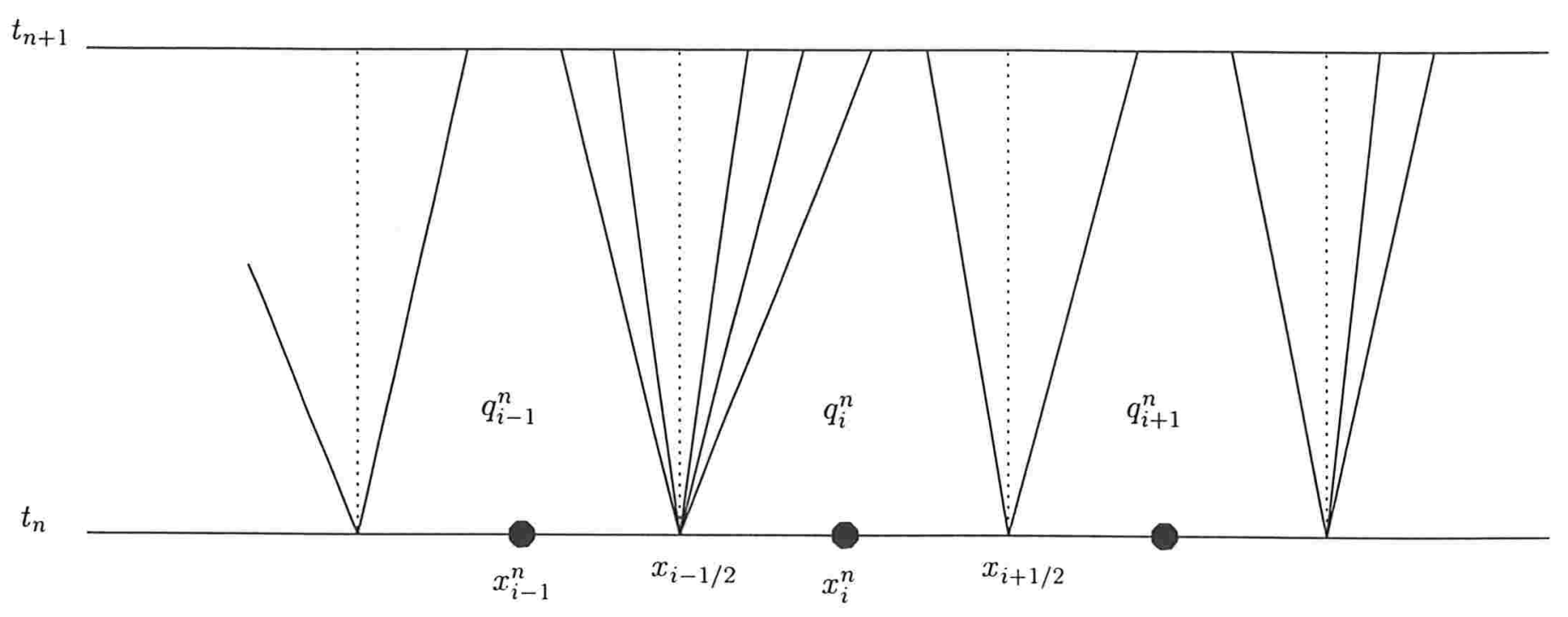

Figura A.1: Solução de problemas de Riemann independentes para dados constantes por partes

Na prática este algoritmo é consideravelmente simplificado, pois a célula média (A.1) pode ser facilmente calculada usando a forma integral da lei de conservação, desde que $\tilde{q}^{n}$ é assumida para ser uma solução fraca exata,

$\int_{x_{i-1 / 2}}^{x_{i+1 / 2}} \tilde{q}^{n}\left(x, t_{n+1}\right) d x=\int_{x_{i-1 / 2}}^{x_{i+1 / 2}} \tilde{q}^{n}\left(x, t_{n}\right) d x+\int_{t_{n}}^{t_{n+1}} f\left(\tilde{q}^{n}\left(x_{i-1 / 2}, t\right)\right) d t-\int_{t_{n}}^{t_{n+1}} f\left(\tilde{q}^{n}\left(x_{i+1 / 2}, t\right)\right) d t$.

Dividindo por $h$, usando a equação (A.1), e observando que $\tilde{q}^{n}\left(x, t_{n}\right) \equiv q_{i}^{n}$ sobre a célula $\left(x_{i-1 / 2}, x_{i+1 / 2}\right)$, esta equação reduz-se para

$$
q_{i}^{n+1}=q_{i}^{n}-\frac{k}{h}\left[F\left(q_{i}^{n}, q_{i+1}^{n}\right)-F\left(q_{i-1}^{n}, q_{i}^{n}\right)\right],
$$

onde a função de fluxo numérica $F$ é dada por

$$
F\left(q_{i}^{n}, q_{i+1}^{n}\right)=\frac{1}{k} \int_{t_{n}}^{t_{n+1}} f\left(\tilde{q}^{n}\left(x_{i+1 / 2}, t\right)\right) d t .
$$

Isto mostra que o Método de Godunov pode ser escrito em forma de conservação. Mais ainda, observemos que a integral necessária para calcular (A.4) é trivial, pois $\tilde{q}^{n}$ é constante no ponto $x_{i+1 / 2}$ sobre o intervalo de tempo $\left(t_{n}, t_{n+1}\right)$. Isto segue do fato que a solução do problema de Riemann em $x_{i+1 / 2}$ é uma solução de similaridade, constante ao longo de cada 
raio $\left(x-x_{i+1 / 2}\right) / t=$ cte. Ver cap.8 de LeVeque [18]. Para o caso escalar, ver seção 2.7 do capítulo anterior.

O valor constante de $\tilde{q}^{n}$ ao longo da linha $x=x_{i+1 / 2}$ depende somente do dado $q_{i}^{n}$ e $q_{i+1}^{n}$ para este problema de Riemann. Se denotamos este valor por $q^{*}\left(q_{i}^{n}, q_{i+1}^{n}\right)$, então o fluxo (A.4) reduz-se para

$$
F\left(q_{i}^{n}, q_{i+1}^{n}\right)=f\left(q^{*}\left(q_{i}^{n}, q_{i+1}^{n}\right)\right),
$$

e o método de Godunov toma a forma

$$
q_{i}^{n+1}=q_{i}^{n}-\frac{k}{h}\left[f\left(q^{*}\left(q_{i}^{n}, q_{i+1}^{n}\right)\right)-f\left(q^{*}\left(q_{i-1}^{n}, q_{i}^{n}\right)\right)\right] .
$$

Este método e todas suas generalizações e extensões são chamados métodos tipo-Godunov.

Quando aplicamos o método de Godunov para equações não lineares escalares, obtemos generalizaçōes do Método Upwind para o caso quando $f^{\prime}(q)$ varia. Como já vimos no capítulo 2 , problemas de Riemann com dados $q_{i+1}^{n}, q_{i}^{n}$ sempre tem uma solução fraca que consiste de uma descontinuidade propagante com velocidade $s=\left(f\left(q_{i+1}^{n}\right)-f\left(q_{i}^{n}\right)\right) /\left(q_{i+1}^{n}-q_{i}^{n}\right)$. Se sempre usamos esta solução de Riemann (que pode não satisfazer a condição de entropia), então o valor intermédio $q^{*}$ na solução do problema de Riemann é dado por

$$
q^{*}\left(q_{i}^{n}, q_{i+1}^{n}\right)=\left\{\begin{array}{lll}
q_{i}^{n} & \text { se } & s>0 \\
q_{i+1}^{n} & \text { se } & s<0 .
\end{array}\right.
$$

Se $s=0$ então este valor não esta bem definido, mas observemos que neste caso $f\left(q_{i}^{n}\right)=f\left(q_{i+1}^{n}\right)$ e portanto o fluxo resultante, que requerimos no método de Godunov, é o mesmo se definimos $q^{*}=q_{i}^{n}$ ou $q^{*}=q_{i+1}^{n}$.

De (A.6) vemos que podemos definir a função de fluxo por

$$
\begin{aligned}
F\left(q_{i}^{n}, q_{i+1}^{n}\right) & =f\left(q^{*}\left(q_{i}^{n}, q_{i+1}^{n}\right)\right) \\
& =\left\{\begin{array}{lll}
f\left(q_{i}^{n}\right) & \text { se } & \left(f\left(q_{i+1}^{n}\right)-f\left(q_{i}^{n}\right)\right) /\left(q_{i+1}^{n}-q_{i}^{n}\right) \geq 0, \\
f\left(q_{i+1}^{n}\right) & \text { se }\left(f\left(q_{i+1}^{n}\right)-f\left(q_{i}^{n}\right)\right) /\left(q_{i+1}^{n}-q_{i}^{n}\right)<0 .
\end{array}\right.
\end{aligned}
$$

O método com esta função de fluxo pode conduzir a soluções que violam a condição de entropia. Para um exemplo ver LeVeque [18]. 
Na implementação do método de Godunov devemos usar soluções que satisfaçam a condição de entropia, esta pode consistir de ondas de rarefação ou ondas de choque. No caso que a função de fluxo $f$ seja convexa, existem quatro casos que devemos considerar :

1. $f^{\prime}\left(q_{i}^{n}\right), f^{\prime}\left(q_{i+1}^{n}\right) \geq 0 \quad \Longrightarrow \quad q^{*}=q_{i}^{n}$,

2. $f^{\prime}\left(q_{i}^{n}\right), f^{\prime}\left(q_{i+1}^{n}\right) \leq 0 \quad \Longrightarrow \quad q^{*}=q_{i+1}^{n}$,

3. $f^{\prime}\left(q_{i}^{n}\right) \geq 0 \geq f^{\prime}\left(q_{i+1}^{n}\right) \quad \Longrightarrow \quad q^{*}=q_{i}^{n}$ se $[f] /[q]>0$ ou $q^{*}=q_{i+1}^{n}$ se $[f] /[q]<0$,

4. $f^{\prime}\left(q_{i}^{n}\right)<0<f^{\prime}\left(q_{i+1}^{n}\right) \quad \Longrightarrow q^{*}=q_{s}$ rarefação transónica.

Em cada um dos três primeiros casos, o valor de $q^{*}$ é $q_{i}^{n}$ ou $q_{i+1}^{n}$ e o fluxo é corretamente dado por (A.7). Observe que nos casos 1 ou 2 é irrelevante se a solução é uma onda de choque ou uma onda de rarefação, desde que o valor de $q^{*}$ é o mesmo em ambos casos.

Somente no caso 4, a rarefação transónica, que o valor de $F$ é diferente de (A.6). Neste caso $q^{*}$ não é nem $q_{i}^{n}$ nem $q_{i+1}^{n}$, mas é o valor intermédio $q_{s}$ com a propriedade que

$$
f^{\prime}\left(q_{s}\right)=0 .
$$

Este é o valor de $q$ para o qual a velocidade da característica é zero, e é chamado ponto sónico.

Podemos modificar (A.7) para incluir esta posibilidade: se $f^{\prime}\left(q_{i}^{n}\right)<0<f^{\prime}\left(q_{i+1}^{n}\right)$ então redefinimos

$$
\left.F\left(q_{i}^{n}, q_{i+1}^{n}\right)\right)=f\left(q_{s}\right) .
$$

A função de fluxo pode ser escrita em uma forma simplificada como

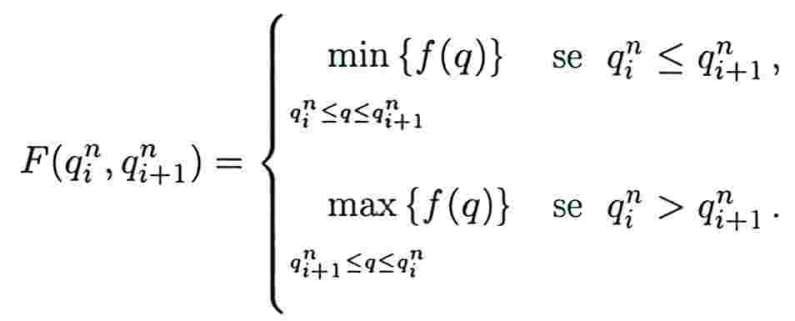




\section{Apêndice B}

\section{B.1 CLAWPACK em duas dimensões}

Em duas dimensões temos a lei de conservação

$$
q_{t}+(f(q))_{x}+(g(q))_{y}=0 .
$$

Discretizaremos nosso problema usando uma malha uniforme com espaçamentos $\Delta x$ e $\Delta y$. Seja $C_{i j} \equiv\left[x_{i-1 / 2}, x_{i+1 / 2}\right] \times\left[y_{j-1 / 2}, y_{j+1 / 2}\right]$ a célula $(i, j)$ da malha com centro em $\left(x_{i}, y_{j}\right)=$ $(i \Delta x, j \Delta y)$ e denotaremos por $q_{i j}^{n}$ o valor médio da célula $(i, j)$ no $n$-ésimo nível de tempo $t_{n}$, isto é,

$$
q_{i j}^{n}=\frac{1}{\Delta x \Delta y} \int_{C_{i j}} q\left(x, y, t_{n}\right) d x d y .
$$

Um método de volume finito conservativo na forma de diferenças de fluxos é da forma

$$
q_{i j}^{n+1}=q_{i j}^{n}-\frac{\Delta t}{\Delta x}\left(F_{i+1, j}^{n}-F_{i j}^{n}\right)-\frac{\Delta t}{\Delta y}\left(G_{i, j+1}^{n}-G_{i j}^{n}\right),
$$

onde $F_{i j}^{n}$ é o fluxo numérico na face esquerda da célula $(i, j)$ e $G_{i j}^{n}$ é o fluxo na sua face inferior, dados, por exemplo, por

$$
F_{i j}^{n}=\frac{1}{\Delta y \Delta t} \int_{t_{n}}^{t_{n+1}} \int_{y_{j-1 / 2}}^{y_{j+1 / 2}} f\left(q\left(x_{i-1 / 2}, y, t\right)\right) d y d t
$$

onde $\left(x_{i-1 / 2}, y_{j-1 / 2}\right)$ é a coordenada do canto inferior esquerdo da célula $(i, j)$, veja a Figura B.1,

O algoritmo de propagação de ondas multi-dimensional usado no CLAWPACK pode ser escrito nesta forma quando é aplicado a uma lei de conservação da forma (B.1), mas é implementado em uma forma mais geral que permite sua aplicação a outros problemas hiperbólicos 


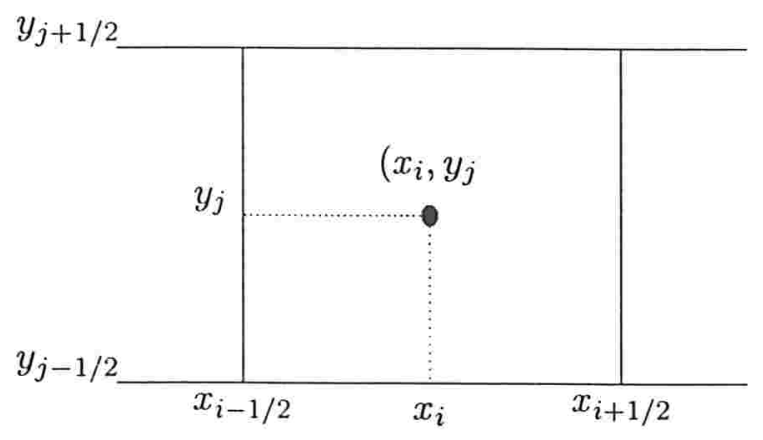

Figura B.1: Discretização do problema.

que não estão em forma conservativa. O método é implementado como

$$
q_{i j}^{n+1}=q_{i j}^{n}+\Delta_{i j}^{\mathrm{up}}-\frac{\Delta t}{\Delta x}\left(\tilde{F}_{i+1, j}^{n}-\tilde{F}_{i j}^{n}\right)-\frac{\Delta t}{\Delta y}\left(\tilde{G}_{i, j+1}^{n}-\tilde{G}_{i j}^{n}\right),
$$

onde os dois primeiros termos correspondem ao Método Upwind de primeira ordem, com

$$
\Delta_{i j}^{\mathrm{up}}=-\frac{\Delta t}{\Delta x}\left(\mathcal{A}^{+} \Delta q_{i j}+\mathcal{A}^{-} \Delta q_{i+1, j}\right)-\frac{\Delta t}{\Delta y}\left(\mathcal{B}^{+} \Delta q_{i j}+\mathcal{B}^{-} \Delta q_{i, j+1}\right) .
$$

Os termos $\mathcal{A}^{ \pm} \Delta q$ e $\mathcal{B}^{ \pm} \Delta q$ representam as diferenças de fluxo originadas dos problemas de Riemann nas direções $x$ e $y$ respectivamente. Os fluxos $\tilde{F}$ e $\tilde{G}$ são usados para realizar as correções de segunda ordem e, também, correções para os termos de derivadas cruzadas que originam-se em duas dimensões, os quais não aparecem em uma dimensão.

$\mathrm{Na}$ descrição que daremos a seguir, primeiramente resolveremos o problema de Riemann na direção- $x$, na interface entre as células $(i-1, j)$ e $(i, j)$ e logo veremos de que forma as ondas do problema de Riemann contribuem para $\Delta_{i j}^{\mathrm{up}}, \tilde{F_{i j}}$ e $\tilde{G_{i j}}$. Um procedimento análogo é seguido para cada interface na direção- $y$ entre as células $(i, j-1)$ e $(i, j)$, comutando os papeis de $F$ e $G$, e $\mathcal{A} \Delta q$ e $\mathcal{B} \Delta q$. A seguir $\Delta q_{i j}$ representará a diferença $q_{i j}^{n}-q_{i-1, j}^{n}$, na direção $x$.

\section{B.1.1 Método de Godunov de Primeira ordem}

Começaremos resolvendo um problema de Riemann unidimensional, normal a cada interface da célula, exatamente como é feito em uma dimensão. Resolvendo o problema de Riemann $q_{t}+f(q)_{x}=0$ com dados $q_{i-1, j}$ e $q_{i j}$, obtemos um conjunto de $M$ ondas e velocidades, com uma decomposição da diferença de fluxos $f\left(q_{i-1, j}\right)-f\left(q_{i j}\right)$ em duas partes $\mathcal{A}^{-} \Delta q_{i j}$ e $\mathcal{A}^{+} \Delta q_{i j}$ 
movendo-se para a esquerda e direita, respectivamente.

Novamente, um resolvedor de Riemann aproximado poderia ser usado (por exemplo, o resolvedor de Roe [27]) para obter as ondas $\mathcal{W}_{i j}^{p}(p=1,2, \ldots, M)$ determinada pelos dados $q_{i-1, j}$ e $q_{i j}$.

As diferenças de fluxo podem novamente ser definidas como

$$
\begin{gathered}
\mathcal{A}^{+} \Delta q_{i j}=A_{i j}^{+}\left(q_{i j}-q_{i-1, j}\right)=\sum_{p}\left(\lambda_{i j}^{p}\right)^{+} \mathcal{W}_{i j}^{p} \\
\mathcal{A}^{-} \Delta q_{i j}=A_{i j}^{-}\left(q_{i j}-q_{i-1, j}\right)=\sum_{p}\left(\lambda_{i j}^{p}\right)^{-} \mathcal{W}_{i j}^{p},
\end{gathered}
$$

com, talvez, uma entropia fixa. Onde $A_{i j}=A_{i j}^{+}+A_{i j}^{-}$é a matriz do problema de Riemann linearizado. Observemos que, com base nas expressões acima, podemos definir um fluxo numérico como

$$
F_{i j}=f\left(q_{i-1, j}\right)+\mathcal{A}^{-} \Delta q_{i j}
$$

ou

$$
F_{i j}=f\left(q_{i-1, j}\right)-\mathcal{A}^{+} \Delta q_{i j}
$$

Estas duas formas, ao igual que numa dimensão, dão o mesmo resultado sempre que

$$
\mathcal{A}^{-} \Delta q_{i j}+\mathcal{A}^{+} \Delta q_{i j}=f\left(q_{i j}\right)-f\left(q_{i-1, j}\right)
$$

Um método tipo Godunov de primeira ordem poderia então ser definido por (B.2), depois de calcularmos os fluxos $G_{i j}$ resolvendo o problema de Riemann para $q_{t}+g(q)_{y}=0$ com dados $q_{i, j-1}$ e $q_{i j}$. Na prática, porém, o método é implementado fazendo-se

$$
\Delta_{i j}^{\mathrm{up}}=-\frac{\Delta t}{\Delta x}\left(\mathcal{A}^{+} \Delta q_{i j}+\mathcal{A}^{-} \Delta q_{i+1, j}\right)-\frac{\Delta t}{\Delta y}\left(\mathcal{B}^{+} \Delta q_{i j}+\mathcal{B}^{-} \Delta q_{i, j+1}\right)
$$

com $\tilde{F} \equiv \tilde{G} \equiv 0$ em (B.4). Aqui $\mathcal{B}^{ \pm} \Delta q_{i j}$ representa uma decomposição da diferença de fluxos $g\left(q_{i j}\right)-g\left(q_{i, j-1}\right)$ que resulta de resolver o problema de Riemann na direção- $y$.

\section{B.1.2 Propagação transversal}

O Método de Godunov descrito acima baseia-se na propagação de ondas normais a cada interface da célula. Na verdade, as ondas deveriam propagar-se de forma multi-dimensional e afetar 
outras células, aquelas adjacentes à interface. Isto é realizado dividindo cada diferença de fluxos $\mathcal{A}^{*} \Delta q_{i j}$, para "*" "+" ou "-" em duas diferenças de fluxos transversais que serão chamados $\mathcal{B}^{+} \mathcal{A}^{*} \Delta q_{i j}$ (a parte indo para acima) e $\mathcal{B}^{-} \mathcal{A}^{*} \Delta q_{i j}$ (a parte indo para baixo).

Na Figura B.2-(a) mostramos um exemplo para a equação de adveç̧ão

$$
q_{t}+u q_{x}+v q_{y}=0,
$$

com velocidades $u, v>0$. Neste caso a onda simples propaga-se na direção $(u, v)$. Existe, nesta figura, uma porção triangular da onda que move-se dentro da célula $(i, j+1)$ em lugar de moverse na célula $(i, j)$. Isto pode ser realizado modificando o fluxo $\tilde{G}_{i, j+1}$ na interface entre estas duas células pela quantidade apropriada,

$$
\tilde{G}_{i, j+1}^{n}:=\tilde{G}_{i, j+1}^{n}-\frac{1}{2} \frac{\Delta t}{\Delta x} u v\left(q_{i j}^{n}-q_{i-1, j}^{n}\right) .
$$

Isto é discutido com mais detalhe, para a equação de advecção em LeVeque [20].

Isto pode ser generalizado para um sistema de equações observando que, para advecção, $u\left(q_{i j}^{n}-q_{i-1, j}^{n}\right)$ é a diferença de fluxos indo para a direita (o qual é toda a diferença de fluxos no caso escalar) e que isto seria propagado para acima pela velocidade vertical $v$. A quantidade $v u\left(q_{i j}^{n}-q_{i-1, j}^{n}\right)$, o produto desta diferença de fluxos e a velocidade vertical, será chamado a diferença de fluxos transversais.

Para um sistema de equações, tipicamente temos uma diferença de fluxos indo para a esquerda $\mathcal{A}^{-} \Delta q_{i j}$ e uma diferença de fluxos indo para a direita $\mathcal{A}^{+} \Delta q_{i j}$. Cada uma destas será decomposta em uma diferença de fluxos indo para acima e uma diferença de fluxos indo para baixo; portanto, existirão quatro diferenças de fluxos modificando os quatro fluxos vizinhos $G$, como indicados na Figura B.2-(b).

A notação $\mathcal{B}^{ \pm} \mathcal{A}^{*} \Delta q_{i j}$ é motivada pelo caso de um sistema linear de equações

$$
q_{t}+A q_{x}+B q_{y}=0 .
$$

Neste caso, as matrizes $B^{ \pm}$são definidas em forma análoga a $A^{ \pm}$, baseados nos autovalores positivos e negativos de $B$. As diferenças de fluxos transversais são então dadas por

$$
\mathcal{B}^{ \pm} \mathcal{A}^{*} \Delta q_{i j}=B^{ \pm} A^{*}\left(q_{i j}-q_{i-1, j}\right)
$$




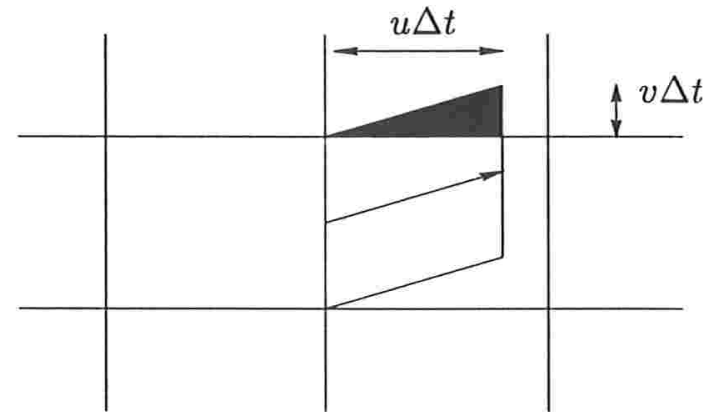

(a)

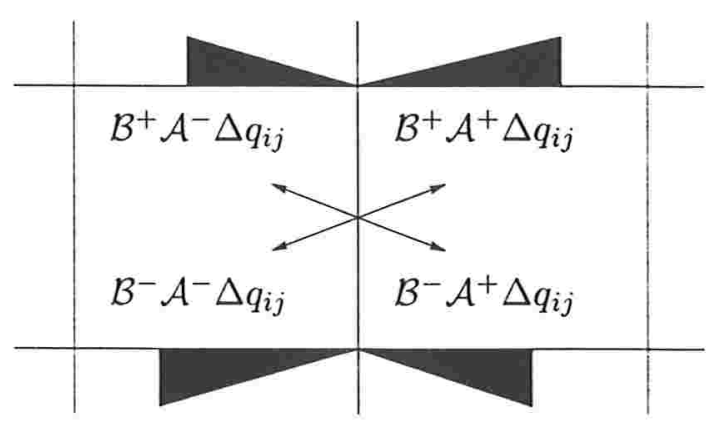

(b)

Figura B.2: (a)-Propagação transversal na equação de advecção. (b)-As quatro diferenças de fluxos transversais para um sistema geral de equações.

onde "*" é ou "+" ou "-".

Em geral, as diferenças de fluxos transversais são usadas para modificar os quatro fluxos vizinhos de acordo com

$$
\begin{array}{rll}
\tilde{G}_{i, j+1} & :=\tilde{G}_{i, j+1}-\frac{1}{2} \frac{\Delta t}{\Delta x} \mathcal{B}^{+} \mathcal{A}^{+} \Delta q_{i j} \\
\tilde{G}_{i, j} & :=\tilde{G}_{i, j}-\frac{1}{2} \frac{\Delta t}{\Delta x} \mathcal{B}^{-} \mathcal{A}^{+} \Delta q_{i j} \\
\tilde{G}_{i-1, j+1} & :=\tilde{G}_{i-1, j+1}-\frac{1}{2} \frac{\Delta t}{\Delta x} \mathcal{B}^{+} \mathcal{A}^{-} \Delta q_{i j} \\
\tilde{G}_{i-1, j} & := & \tilde{G}_{i-1, j}-\frac{1}{2} \frac{\Delta t}{\Delta x} \mathcal{B}^{-} \mathcal{A}^{-} \Delta q_{i j}
\end{array}
$$

Para um sistema linear (B.11), a soma de todas as diferenças de fluxos transversais é

$$
\left(B^{+} A^{+}+B^{-} A^{+}+B^{+} A^{-}+B^{-} A^{-}\right) \Delta q=B A \Delta q .
$$

Como $\Delta q$ representa uma diferença na direção- $x$, e os fluxos $G$ são diferenças de fluxos na direção-y, atualizando $q$, (B.12) resulta numa aproximação para $\frac{1}{2} \Delta t B A q_{x y}$, que é um dos termos das derivadas cruzadas necessárias para atingir precisão de segunda ordem.

Observemos que para a equação linear de advecção (B.10), temos,

$$
\begin{array}{ll}
\mathcal{B}^{+} \mathcal{A}^{+} \Delta q_{i j}=v^{+} u^{+} \Delta q, & \mathcal{B}^{+} \mathcal{A}^{-} \Delta q_{i j}=v^{+} u^{-} \Delta q \\
\mathcal{B}^{-} \mathcal{A}^{+} \Delta q_{i j}=v^{-} u^{+} \Delta q, & \mathcal{B}^{-} \mathcal{A}^{-} \Delta q_{i j}=v^{-} u^{-} \Delta q
\end{array}
$$


e desde que $u$ e $v$ são ambos positivos, somente a primeira destas quatro diferenças de fluxos é diferente de zero. Portanto, somente o fluxo na direção de advecção é modificado pela quantidade total $v u \Delta q$, com contribuição zero nas outras três direções.

Para um sistema linear de equações, quando $A$ e $B$ são simultaneamente diagonalizáveis (isto é, têm os mesmos autovetores), o sistema pode ser transformado em um conjunto de $m$ equações de advecção escalar independentes e a escolha (B.13) é equivalente a aplicar o algoritmo de advecção para cada uma destas equações independentes.

Mesmo que $A$ e $B$ não sejam simultaneamente diagonalizáveis, as diferenças de fluxos transversais podem também ser interpretadas em termos de propagação de ondas multi-dimensionais. A diferença de fluxos $\mathcal{A}^{+} \Delta q$, por exemplo, é a soma de $\lambda^{P} \mathcal{W}^{p}$ de todas as ondas indo para a direita. Se decompusermos cada onda $\mathcal{W}^{p}$ como uma combinação linear de autovetores de $B$,

$$
\mathcal{W}^{p}=\sum_{s=1}^{m} \beta^{p s} w^{s}
$$

onde $B w^{s}=\mu^{s} w^{s}$, então a sub-onda $\beta^{p s} w^{s}$ será propagada para acima ou para abaixo com velocidade $\mu^{s}$, dependendo de se $\mu^{s}$ é positivo ou negativo. O fluxo indo para acima, por exemplo, é

$$
\sum_{\mu^{s}>0} \mu^{s} \beta^{p s} w^{s}
$$

Somando todas estas ondas, indo para a direita, $\mathcal{W}^{p}$ para $\lambda^{p}>0$ resulta em

$$
\sum_{\lambda^{p}>0} \lambda^{p} \sum_{\mu^{s}>0} \mu^{s} \beta^{p s} w^{s},=\sum_{\mu^{s}>0}\left(\sum_{\lambda^{p}>0} \lambda^{p} \beta^{p s}\right) w^{s},
$$

o qual é precisamente $B^{+} A^{+} \Delta q$. As outras diferenças de fluxos transversais podem ser interpretadas similarmente como combinações de ondas nas outras direções.

Para um sistema não linear de equações, necessitamos decompor $\mathcal{A}^{*} \Delta q_{i j}$, onde "*" representa "+" ou "-", empregando os autovetores de alguma matriz Jacobiana $g^{\prime}(q)$ ou o Jacobiano aproximado perto dos estados $q_{i-1, j}$ e $q_{i j}$. Se chamarmos este Jacobiano aproximado de $B_{i j}$, então podemos simplesmente multiplicar $\mathcal{A}^{*} \Delta q_{i j}$ por $B_{i j}^{+}$e $B_{i j}^{-}$para definir $\mathcal{B}^{+} \mathcal{A}^{*} \Delta q_{i j}$ e $\mathcal{B}^{-} \mathcal{A}^{*} \Delta q_{i j}$.

Em particular, se estivermos usando um resolvedor de Riemann aproximado (por exemplo, o resolvedor de Roe [27]) então podemos usar as quantidades médias-Roe necessárias na definição 
desta matriz para também definir a matriz $B_{i j}$ (o mais precisamente, os autovetores e autovalores que são tipicamente usados diretamente sem ainda formar $B_{i j}$ ).

\section{B.1.3 Correções de segunda ordem e sua propagação transversal}

Uma vez que as correções transversais descritas acima tenham sido implementadas, é possível atingir precisão de segunda ordem simplesmente fazendo correções de fluxo unidimensional análogas a (3.12). É neste ponto que as ondas $\mathcal{W}_{i j}^{p}$ e velocidades $\lambda_{i j}^{p}$ são usadas. Fazemos as correções

$$
\tilde{F}_{i j}^{n}:=\tilde{F}_{i j}^{n}+\frac{1}{2} \sum_{p=1}^{m}\left|\lambda_{i j}^{p}\right|\left(1-\frac{\Delta t}{\Delta x}\left|\lambda_{i j}^{p}\right|\right) \tilde{\mathcal{W}}_{i j}^{p},
$$

onde $\tilde{W}_{i j}^{p}$ é uma versão limitada de $\mathcal{W}_{i j}^{p}$. O limitador é aplicado exatamente como em uma dimensão, portanto $\mathcal{W}_{i j}^{p}$ é comparado com $\mathcal{W}_{i-1, j}^{p}$ ou $\mathcal{W}_{i+1, j}^{p}$ dependendo se $\lambda_{i j}^{p}>0$ ou $<0$.

O método acima já é de segunda ordem, mas é fácil também propagar as correções de segunda ordem na direção transversal. Isto é motivado pelo "Método 4" de LeVeque [20] e, se por um lado é verdade que estas correções adicionais não incrementam a ordem de precisão, por outro lado elas melhoram as propriedades de estabilidade e reduzem as oscilações em muitos problemas.

As correções do fluxo (B.14) afetarão $q_{i-1, j}^{n}$ e $q_{i j}^{n}$ nas células à esquerda e à direita desta interface. Portanto, a propagação transversal desta correção afetará os quatro fluxos $G$, aqueles abaixo e acima destas duas células.

As correções poderíam ser divididas em duas partes, uma indo para cima e a outra indo para baixo, exatamente na mesma forma como $\mathcal{A}^{*} \Delta q$ são divididas em $\mathcal{B}^{+} \mathcal{A}^{*} \Delta q_{i j}$ e $\mathcal{B}^{-} \mathcal{A}^{*} \Delta q_{i j}$. Mas, na verdade, o algoritmo com esta propagação transversal é implementada simplesmente modificando $\mathcal{A}^{*} \Delta q$ com estas correções de segunda ordem, chamando a rotina que divide estes vetores em forma transversal. As modificações serão

$$
\begin{aligned}
& \mathcal{A}^{+} \Delta q:=\mathcal{A}^{+} \Delta q-\frac{1}{2} \sum_{p=1}^{m} \mid \lambda_{i j}^{p}\left(1-\frac{\Delta t}{\Delta x}\left|\lambda_{i j}^{p}\right|\right) \tilde{\mathcal{W}}_{i j}^{p}, \\
& \mathcal{A}^{-} \Delta q:=\mathcal{A}^{-} \Delta q+\frac{1}{2} \sum_{p=1}^{m} \mid \lambda_{i j}^{p}\left(1-\frac{\Delta t}{\Delta x}\left|\lambda_{i j}^{p}\right|\right) \tilde{\mathcal{W}}_{i j}^{p} .
\end{aligned}
$$


Observemos que o termo corretor $\frac{1}{2} \sum \ldots$ é exatamente o mesmo que o termo que modifica $\tilde{F}_{i j}$ em (B.14). 


\section{Referências Bibliográficas}

[1] M. J. Berger, Adaptive mesh refinement for hyperbolic partial differential equations, Ph.D. thesis, Computer Science Department, Stanford University, 1982.

[2] — Adaptive finite difference methods in fluid dynamics, Von Karman Institute for Fluid Dynamics Lecture Series (1987).

[3] — On conservation at grid interfaces, SIAM J. Num. Anal. 24 (1987), 967--

[4] M. J. Berger e P. Colella, Local adaptive mesh refinement for shock hydrodynamics, $J$. Comput. Phys. 82 (1989), 64-84.

[5] M. J. Berger e R. J. LeVeque, Adaptive mesh refinement using wave-propagation algorithms for hyperbolic systems, SIAM J. Numer. Anal. 35 (1998), 2298-2316.

[6] M. J. Berger e J. Oliger, Adaptive mesh refinement for hyperbolic partial differential equations, J. Comput. Phys. 53 (1984), 484-512.

[7] A. Bourlioux, Numerical study of unstable detonations, Ph.D. thesis, Princeton University, June, 1991.

[8] R. Courant e K. O. Friedrichs, Supersonic flow and shock waves, Wiley-Interscience:New York, 1948.

[9] J. B. Goodman e R. J. LeVeque, A geometric approach to high resolution tvd schemes, SIAM J. Num. Anal. 25 (1988), 268-284.

[10] E. Hopf, A partial differential equations $u_{t}+u u_{x}=\mu u_{x x}$, Comm. Pure Appl. Math. 3 (1950), 201-230. 
[11] F. Jhon, Nonlinear wave equations, formation of singularities, 1990, Revised notes of the Seventh Annual Pitcher Lectures in 1989.

[12] H. O. Kreiss e J. Lorenz, Inicial-boundary value problems and the navier-stokes equations, Academic Press, 1989.

[13] P. D. Lax, Hyperbolic systems of conservation laws, (1957), no. 10, 537-566.

[14] _ Hyperbolic systems of conservation laws and the mathematical theory of shock waves, SIAM Regional Conference Series in Applied Mathematics, no. 11, 1972.

[15] R. J. LeVeque, Clawpack software, http://www.amath.washington.edu/ rjl/clawpack.html.

[16] _ Intermediate boundary conditions for time-split methods applied to hyperbolic partial differential equations, 47 (1986), 37-54.

[17] _ High resolution finite volume methods on arbitrary grids, 78 (1988), 36-63.

[18] _ Numerical methods for conservation laws, 2end ed., Lectures in Mathematics ETH Zürich, 1992.

[19] — Numerical Methods for Fluid Dynamics, ch. 4, pp. 175-190, Oxford University Press, 1993.

[20] — High-resolution conservative algorithms for advection in incompressible flow, 33 (1996), 627-665.

[21] - Wave propagation algorithms for multidimensional hyperbolic system, J. Comput. Phys. 131 (1997), 327-353.

[22] R. J. LeVeque e K. M. Shyue, One-dimensional front tracking based on high resolution wave propagation methods, 16 (1995), 348-377.

[23] R. J. LeVeque e H. C. Yee, A study of numerical methods for hyperbolic conservation laws with stiff source terms, J. Comput. Phys. 86 (1990), 187-210.

[24] O. A. Oleinik, Discontinuous solutions of nonlinear differential equations, Amer. Math. Soc. Transl. 26 (1957), 95-172.

[25] R. B. Pember, Numerical methods for hyperbolic conservation laws with stiff relaxation, ii. higher order godunov methods, SIAM J. Sci. Comput. 14 (1993), 327-353. 
[26] M. Renardy e R. C. Rogers, An introduction to partial differential equations, Text in Applied Mathematics (TAM), no. 13, Springer-Verlag, 1993.

[27] R. Roe, Approximate riemann solvers, parameter vectors, and difference schemes, J. Computational Physics 45 (1981), 357-372.

[28] J. Smoller, Shock waves and reaction-difussion equations, 2end ed., Comprehensive Studies in Mathematics, no. 258, Springer-Verlag, 1994.

[29] G. Strang, On the construction and comparisom of difference schemes, SIAM J. Num. Anal. 5 (1968), 506-517.

[30] P. K. Sweby, High resolutions schemes using flux limiters for hyperbolic conservation laws, SIAM J. Num. Anal. 21 (1984), 995-1011.

[31] A. I. Vol'pert, The space $b v$ and quasilinear equations, Math. USSR-Sbornik 2 (1967), 225-267. 